\title{
Classes of Valuations Closed under Operations Galois-dual to Boolean Sentence Connectives
}

By

\author{
Lloyd HUMBERSTONE*
}

\section{Contents}

\section{Outline}

8 0. Introduction and Background

0.1. Closure and Generalized Closure

0.2. Galois Connexions

0.3. Combinations on Both Sides of a Galois Connexion

0.4. The Exchange Property

0.5. Two Auxiliary Notations

0.6. Tight Sets and the Exchange Property

$\S 1$. Supervenience and Matching

1.1. Two Problems Suggested by the Idea of Supervenience

1.2. (Flip-Around) and Some Related Conditions

$\S 2$. Closure under Operations Galois-Dual to Various Boolean Connectives

2.1. Negative Objects

2.2. Disjunctive Combinations

2.3. Conjunctive Combinations

2.4. Implicative Combinations

2.5. Some Further Cases

2.6. The Provenance of the Conditions on Generalized Closure Relations

§ 3. Determination by Classes of Valuations Closed under Matching

3.1. An Approach Suggested by the Preceding Discussion

References

3.2. An Unanswered Question and a Further Condition

\section{Outline}

Where $U$ is a language and $\mathscr{V}$ is a class of valuations (truth-value assignments to the formulas of $U$ ), compare the hypotheses

(†) $\forall x_{1}, x_{2} \in U \exists x_{3} \in U \forall v \in \mathscr{V}: v\left(x_{3}\right)=\mathrm{T}$ iff $v\left(x_{1}\right)=\mathrm{T}$ or $v\left(x_{2}\right)=\mathrm{T}$

(††) $\forall v_{1}, v_{2} \in \mathscr{V} \exists v_{3} \in \mathscr{V} \forall x \in U: v_{3}(x)=\mathrm{T}$ iff $v_{1}(x)=\mathrm{T}$ or $v_{2}(x)=\mathrm{T}$.

Communicated by R. Nakajima, February 21, 1994.

1991 Mathematics Subject Classifications: 03B05, 06A15.

* Monash University, Clayton, Victoria 3168, Australia. 
We describe a Galois connexion between $U$ and $\mathscr{V}$ in terms of which $(\dagger)$ and $(\dagger \dagger)$ are Galois-dual statements; the first saying that there are disjunctive combinations on the left (' $U$ ) side of this connexion, and the second saying that there are disjunctive combinations on the right (' $\mathscr{V}$ ') side. Traditionally, sentential logic explores the effect of such assumptions as $(\dagger)$ on the consequence relation (on $U$ ) determined by (= sound and complete w. r. t.) $\mathscr{V}$; for example, writing $x_{1} \vee x_{2}$ for the $x_{3}$ promised by ( $\dagger$ ), we have, for all $x, y, z \in U: x \vee y \vdash y \vee x$; if $x \vdash z$ and $y \vdash z$ then $x \vee y \vdash z$; and so on. We shall open up for exploration in this paper the effect of such assumptions as $(\dagger \dagger)$ on the consequence relation $\vdash$ (on $U$ ) determined by $\mathscr{V}$. These effects include, for example, the principle: If $x, y \vdash z$ then either $x \vdash z$ or $y \vdash z$. We investigate, that is, the logical repercussions of closure assumptions like $(\dagger \dagger)$ on the right, rather than of closure assumptions like $(\dagger)$ on the left. In reasoning about such matters we notate the disjunctive combination of $v_{1}$ and $v_{2}$ (the $v_{3}$ promised by $(\dagger \dagger)$ ) as $v_{1} \nabla v_{2}$, describing $\nabla$ as that operation on valuations which is Galois-dual to the sentence connective $\vee$. Clearly any boolean sentence connective gives rise similarly to a Galois-dual operation on valuations.

Actually the above description is oversimplified in numerous respects, one of which being that we treat the case in which $U$ is a class of formulas as a special case, reserving talk of 'logic' for this case, and instead of talking of consequence relations, which is too suggestive of the special case, we will speak of 'closure relations' on an arbitrary underlying set $U$; the need for this extra generality will be evident from 0.4 and 1.1. The latter passage introduces an example raising a problem in terms of which we have chosen to organize much of the discussion, namely the operation Galois-dual to material equivalence (so that we have the 'or' in $(\dagger \dagger)$ replaced by 'if and only if'); this problem arises naturally from reflection on the philosophical concept of supervenience or on the database-theoretic concept of functional dependency.

\section{$\S 0$. Introduction and Background}

We introduce some concepts and results which will be of use in §§1-3; the discussion often goes somewhat beyond the bare minimum needed by way of such preliminaries when a point of more general interest can conveniently be made in the context of the surrounding preparatory material. Results which are generally known, or are immediate consequences of the definitions given, are stated without proof. A section-by-section summary of the paper-not intelligible until some of those definitions have been provided-may be found toward the end of the final paragraph of 0.2 below. (For a more general overview, see the Outline.)

\subsection{Closure and Generalized Closure}

By a closure operation on some set $U$ we mean a mapping $C: \mathscr{P}(U) \rightarrow \mathscr{P}(U)$ 
such that for all $X, Y \subseteq U$ we have $X \subseteq C(X)=C(C(X))$ and, whenever $X \subseteq Y$, $C(X) \subseteq C(Y)$. We call $U$ the underlying set of $C$; we will always require $U \neq \varnothing$. A closure operation on the set of formulas of some language is usually called a consequence operation; the associated relation $\vdash$ holding between $X \subseteq U$ and $x \in C(X)$, in which case we write $X \vdash x$, satisfies the following conditions of Reflexivity, Monotonicity ('Thinning') and Transitivity ('Cut'), whose labelling here is adapted from $\operatorname{Scott}$ (e.g. [23]). For all $X, Y \subseteq U$, all $x, z \in U$ :

$(\mathbb{R}) x \vdash x$

(M) $X \vdash x$ implies $X, Y \vdash x$

$\left(\mathbb{T}^{+}\right)$If $X \vdash y$ for each $y \in Y$, and $X, Y \vdash z$, then $X \vdash z$.

Some customary notational liberties have been taken here, with ' $x \vdash x$ ' written in place of ' $\{x\} \vdash x$ ' and ' $X, Y \vdash x$ ' for ' $X \cup Y \vdash x$ '; similarly, below, we write such things as ' $X, x \vdash y$ ', ' $\vdash y$ ', for (resp.) ' $X \cup\{x\} \vdash y$ ', ' $\varnothing \vdash y$ '. The consequence operation $C$ can be recovered from $\vdash$ by taking $C(X)=\{u \in U \mid X \vdash u\}$. The consequence operation associated in this way with $\vdash$ will be denoted by $C_{\vdash}$.

Since we prefer not to be restricted to the case in which the underlying sets $U$ are sets of formulas - for more on which restriction, see $0.4-$ we generally use the 'closure operation' terminology rather than the 'consequence operation' terminology. But the relational notation is more convenient in practice (and suggestive of logical analogies) so we need a similarly neutral term here, and accordingly call any relation $\vdash \subseteq \mathscr{P}(U) \times U$ satisfying $(\mathbb{R}),(\mathbb{M}),\left(\mathbb{T}^{+}\right)$above a closure relation on (or 'with underlying set') $U$. When we have in mind cases in which $U$ is, specifically, some set of formulas, we will use the more specific 'consequence' terminology. The same applies in the case of generalized consequence relations on $U$, which is to say relations $\vdash \subseteq \mathscr{P}(U) \times \mathscr{P}(U)$ satisfying analogues of the above three conditions, and for which we use the same labels; again capitals range over arbitrary subsets, and lower case letters $(u, w, x, y, z$ : ' $v$ ' is reserved for another use, introduced in 0.2) over elements, of $U$ :

( $\mathbb{R}) x \vdash x$

(M) $X \vdash W$ implies $X, Y \vdash W, Z$

$\left(\mathbb{T}^{+}\right)$If $X, Y_{0} \vdash Y_{1}, Z$ for all $Y_{0}, Y_{1}$ such that $Y_{1}=Y \backslash Y_{0}$ for some set $Y$, then $X \vdash Z$.

To subsume the non-linguistic cases under our discussion, we call $\vdash$ satisfying these conditions a generalized closure relation (on $U$ ). We use the same notation (' $\vdash$ ') both for closure relations and for generalized closure relations so that some conditions formulated with its aid (namely those in which at exactly one element appears to the right) can be interpreted both as conditions on closure relations and as conditions on generalized closure relations. To negate a claim (under either interpretation) made with the aid of ' $\vdash$ ', we write ' $\forall$ '.

We collect together some further definitions and examples before passing to a semantic description of (generalized) closure relations, beginning with the closed sets for a given closure rlation. A subset $X \subseteq U$ is $\vdash$-closed, for $\vdash$ some closure 
relation on $U$, when for all $u \in U, X \vdash u$ implies $u \in X$. (I.e., when $C_{\vdash}(X)=X$.)

(Note that while there is a reasonable notion of a set $X$ 's being $\vdash$-closed where $\vdash$ is a generalized closure relation (on $U$, with $X \subseteq U$ ), namely when for any $Y \subseteq U$ with $X \vdash Y, X \cap Y \neq \varnothing$, there is no corresponding notion of 'the' generalized closure of a given set $X_{0}$, since there is no least superset $X$ of $X_{0}$ meeting the condition just described.)

By a Lindenbaum closure relation, we shall mean a closure relation $\vdash$ with the property that whenever $X \nvdash u$, there is some $X^{\prime} \supseteq X$ such that $X^{\prime} \nvdash u$ and for any $y \notin X^{\prime}$, we have $X^{\prime}, y \vdash u$. In this case we say that $X^{\prime}$ maximally avoids $u$, and a set which maximally avoids some element of the underlying set $U$ will be called a maximal avoider (relative to $\vdash$ ). It is not hard to see that all maximal avoiders relative to $\vdash$ are $\vdash$-closed sets. (The notion here going under the name 'Lindenbaum closure relation' appears not to have been isolated in print; however, the analogous notion for closure operations appears in the terminology of 'strongly regular' such operations in an unpublished paper, [27], of Stan Surma.)

A closure relation $\vdash$ on $U$ is finitary when for all $X \subseteq U, u \in U$, if $X \vdash u$ then there is some finite $X_{0} \subseteq X$ with $X_{0} \vdash u$, and a generalized closure relation $\vdash$ on $U$ is finitary when for all $X, Y \subseteq U$, if $X \vdash Y$ then there are finite subsets $X_{0}, Y_{0}$ of $X, Y$ respectively, with $X_{0} \vdash Y_{0}$. For a finitary closure relation we can replace the conditon $\left(\mathbb{T}^{+}\right)$above with the simpler condition

(T) If $X \vdash y$ and $X, y \vdash z$, then $X \vdash z$.

And for a finitary generalized closure relation, there is a similar simplification of $\left(\mathbb{T}^{+}\right)$for such relations with

(T) If $X, y \vdash Z$ and $X \vdash y, Z$, then $X \vdash Z$.

(For details see [26], Chapter 2.) Note that these conditions are special cases of the conditions $\left(\mathbb{T}^{+}\right)$on closure relations and generalized closure relations arising in each case by taking $Y$ as $\{y\}$; in what follows we shall mostly need to appeal only to the fact that (generalized) closure relations satisfy, alongside $(\mathbb{R})$ and $(\mathbb{M})$, these weaker conditions $(\mathbb{T})$.

What is often referred to as Lindenbaum's Lemma is the claim that any finitary closure relation is (what we are calling) a Lindenbaum closure relation, or, in the context of discussing a particular such relation, that it is a Lindenbaum closure relation. Though finitariness is sufficient for a closure relation to be a Lindenbaum closure relation, it is not necessary:

Example 0.1.1. Let $U$ be a language with countably many atomic formulas, and closed under the formation rule that if $X$ is any non-empty subset of $U$, then 
$\Lambda X \in U$. (We exclude the case of $X=\varnothing$ for convenience.) Let $\vdash$ be the least closure relation on $U$ satisfying

$$
\begin{aligned}
& X \vdash \Lambda X \text { for all } X \neq \varnothing ; \\
& \Lambda X \vdash x \text { for any } x \in X .
\end{aligned}
$$

Thus $\vdash$ is the usual consequence relation for the logic of infinitary conjunction. Clearly $\vdash$ is not finitary, since (e.g.) if $X$ is the set of all atomic formulas, $X \vdash \Lambda X$ while for no finite $X_{0} \subseteq X$ do we have $X_{0} \vdash \Lambda X$. $\vdash$ is, however, a Lindenbaum closure relation, as we see by the following alternative characterization of $\vdash$. We call $x$ a conjunct of $y(x, y \in U)$ if for some $X$ with $x \in X, y=\Lambda X$; the ancestral (reflexive transitive closure) of this relation we express by saying that $x$ is a conjunct ${ }^{*}$ of $y$. Then it is easily checked that for all $Y \subseteq U, z \in U: Y \vdash z$ just in case every atomic conjunct* of $z$ is a conjunct* of some $y \in Y$. To see that $\vdash$ is a Lindenbaum closure relation, suppose that $Y \nvdash z$; then at least one atomic conjunct* of $z$ is not a conjunct of any $y \in Y$. Let $z_{0}$ be one such atomic conjunct* of $z$ and put

$$
Y^{+}=\left\{u \in U \mid z_{0} \text { is not a conjunct }{ }^{*} \text { of } u\right\} .
$$

$Y^{+}$is then a superset of $Y$ which maximally avoids $z$.

The reason we emphasize the fact that the above Lindenbaum property is strictly weaker than finitariness is that certain results which appear below in $\$ \$ 1-3$ deal with arbitrary Lindenbaum closure relations and we do not wish the degree to which this is a restriction (of the class of all closure relations) to be overstimated.

\subsection{Galois Connexions}

We recall, in a manner tailored to subsequent developments, the 'semantic' aspect of the notions here introduced. A valuation for $U$ is a map from $U$ to the set $\{\mathrm{T}, \mathrm{F}\}$ of truth-values. We will sometimes call valuations for $U$ ' $U$-valuations', and will often omit the reference to $U$ when this is clear from the context. In the case in which $U$ is language - for which case one would normally drop the scare-quotes on "semantic" - such a map classifies formulas into those taken as true and those taken as false; in the general case, a valuation is just the characteristic function of some subset of $U$. Letting $\mathscr{V}$ be any set of valuations on $U$, the relation holding between $u \in U$ and $v \in \mathscr{V}$ when $v(u)=\mathrm{T}$ induces a Galois connexion between $U$ and $\mathscr{V}$ in terms of which we can consider closure relations on $U$; but first, since there are numerous notions of Galois connexion (or Galois correspondence) current in the literature, we make explicit the one we have in mind. (This is the original notion, as found in [20]; for some modern variants, less convenient for present purposes, see pp.93-95 of [18] , and [12]. The main variation, tailored for linking these ideas with adjoints in category theory, is to make the replace the 
'antitone' conditions (G3) and (G4) below, by 'monotone' versions; see also [10], p.29, for further historical and comparative remarks. The reference to $\subseteq$ may also be replaced by one to arbitrary partial orderings $\leq$ on the sets concerned. The content of the next few paragraphs is in pp.121-126 of [2], in a slightly different terminology, as well as in [20].)

Given a pair $L, R$, of sets, a Golois connexion between $L$ ("on the left") and $R$ ("on the right") is a pair of maps $f: \mathscr{P}(L) \rightarrow \mathscr{P}(R), g: \mathscr{P}(R) \rightarrow \mathscr{P}(L)$ satisfying, for all $L_{0}, L_{1} \in L, R_{0}, R_{1} \subseteq R$ :
(G1) $L_{0} \subseteq g\left(f\left(L_{0}\right)\right)$
(G2) $R_{0} \subseteq f\left(g\left(R_{0}\right)\right)$
(G3) $L_{0} \subseteq L_{1}$ implies $f\left(L_{1}\right) \subseteq f\left(L_{0}\right)$
(G4) $R_{0} \subseteq R_{1}$ implies $g\left(R_{1}\right) \subseteq g\left(R_{0}\right)$.

Since interchanging variables ranging over subsets of $L$ with variables ranging over subsets of $R$, and simultaneously interchanging $f$ and $g$, replaces either of (G1), (G2) with the other, and likewise for (G3), (G4), we can regard such interchanges as giving a duality principle: if $(f, g)$ is a Galois connexion between $L$ and $R$ then $(g, f)$ is a Galois connexion between $R$ and $L$ (the 'dual' Galois connexion). Thus having shown on the basis of these four defining conditions, for example, that for all $L_{0}, L_{1} \subseteq L$

$$
f\left(L_{0} \cup L_{1}\right)=f\left(L_{0}\right) \cap f\left(L_{1}\right)
$$

we know that the dual principle, resulting from the indicated interchanges, is also correct, for all $R_{0}, R_{1} \subseteq R$ :

$$
g\left(R_{0} \cup R_{1}\right)=g\left(R_{0}\right) \cap g\left(R_{1}\right)
$$

It is important not to confuse this notion of Galois duality with the usual boolean (lattice-theoretic, or more generally poset-theoretic) duality; unlike (1) and (2), the boolean dual of (1), namely

$$
f\left(L_{0} \cap L_{1}\right)=f\left(L_{0}\right) \cup f\left(L_{1}\right)
$$

does not hold (for all $L_{0}, L_{1} \subseteq L$ ) in every Galois connexion. Below, we shall make a further extension of the notion of Galois duality. With $f, g$ in a Galois connexion as above, the composite maps $f \circ g$ and $g \circ f$ are closure operations on $R$ and $L$ respectively.

As already intimated, the Galois connexions in which we are interested arise for a given $U$ (which we are mentally picturing as "on the left") and various classes $\mathscr{V}$ of valuations for $U$ ("on the right"), and the maps $f$ and $g$ we shall write as $V$ ("verifiers") and $T$ ("truths"), defined as follows, where $X \subseteq U$ and $\mathscr{V}_{0} \subseteq \mathscr{V}$ : 
$V(X)=\{v \in \mathscr{V} \mid v(u)=\mathrm{T}$ for all $u \in X\}$

$$
T\left(\mathscr{V}_{0}\right)=\left\{u \in U \mid v(u)=\mathrm{T} \text { for all } v \in \mathscr{V}_{0}\right\} .
$$

The fact that (G1) - (G4) above are satisfied in this case depends only on the fact that ' $v(u)=\mathrm{T}$ ' appearing in these definitions expresses a binary relation between $U$ and $\mathscr{V}$; that is, for any sets $L$ and $R$ and any relation $\varphi \subseteq L \times R$, setting, for any $L_{0} \subseteq L$

$$
f\left(L_{0}\right)=\left\{r \in R \mid \varphi(l, r) \text { for all } l \in L_{0}\right\}
$$

and, for any $R_{0} \subseteq R$

$$
g\left(R_{0}\right)=\left\{l \in L \mid \varphi(l, r) \text { for all } r \in R_{0}\right\}
$$

make the pair $(f, g)$ a Galois connexion between $L$ and $R$, called the Galois connexion induced by the relation $\varphi$. We can recover $\varphi$ by setting

$$
\varphi(l, r) \text { if and only if } r \in f(\{l\})
$$

or equivalently

$$
\varphi(l, r) \text { if and only if } l \in g(\{r\}) .
$$

The equivalence here observed shows that the identity of $f$ or $g$ is fixed once the other is given, in the sense that if $(f, g)$ and $\left(f, g^{\prime}\right)$ are both Galois connexions between $L$ and $R$, then $g=g^{\prime}$, similarly with $(f, g),\left(f^{\prime}, g\right)$ both being Galois connexions between $L$ and $R$, we must have $f=f^{\prime}$.

The closure operation $T \circ V$ on $U$ is called the closure operation determined by $\mathscr{V}$, and the associated closure relation will be similarly described. Thus for this closure relation $\vdash$ we have, for all $X \subseteq U, u \in U$ :

$$
X \vdash u \text { if and only if } u \in T \circ V(X)=T(V(X)) \text {. }
$$

The right-hand side here can be put alternatively by saying that $V(X) \subseteq V(u)$, where we write ' $V(u)$ ' in place of ' $V(\{u\})$ ', or again, by saying that for every $v \in \mathscr{V}$ with $v(x)=\mathrm{T}$ for all $x \in T, v(u)=\mathrm{T}$. Similarly, we say that a generalized closure relation $\vdash$ (on $U$ ) is determined by a class $\mathscr{V}$ of valuations (for $U$ ) when for all $X, Y \subseteq U: X \vdash Y$ iff for every $v \in \mathscr{V}$ with $v(x)=\mathrm{T}$ for all $x \in X$, we have $v(y)=\mathrm{T}$ for some $y \in Y$. (Another logically significant kind of Galois connexion will be mentioned in the Digression in 0.4.)

Given any closure relation $\vdash$ on $U$, a valuation $v$ for $U$ is consistent with $\vdash$ if there do not exist $X \subseteq U, u \in U$ with $X \vdash u$ while $v(x)=\mathrm{T}$ for all $x \in X$ and $v(u)=\mathrm{F}$; a valuation $v$ is consistent with a generalized closure relation $\vdash$ on $U$ if there do not exist $X, Y \subseteq U$ with $X \vdash Y$ while $v(x)=\mathrm{T}$ for all $x \in X$ and $v(y)=\mathrm{F}$ for all $y \in Y$. (This terminology is taken from [23] ; when considering whether or not a valuation is consistent with $\vdash$ we assume we are dealing with a 
$U$-valuation, where $U$ is the underlying set of $\vdash$.) We denote the class of all valuations consistent with a (generalized) closure relation $\vdash$ by $\mathscr{V}_{a t}(\vdash)$. The following is well known:

Theorem 0.2.1. Any closure or generalized closure relation $\vdash$ is determined by $\mathscr{V a t}(\vdash)$.

An important difference between closure relations and generalized closure relations is that for a generalized closure relation $\vdash$, the only class of valuations which determines $\vdash$ is $\mathscr{V}_{a}(\vdash)$, while a closure relation can be determined not only by the class of all its consistent valuations but by many proper subsets thereof. For the former point, suppose that $\mathscr{V}$ and $\mathscr{V}^{\prime}$ are classes of valuations for $U$, the underlying set of generalized consequence relations $\vdash$ and $\vdash^{\prime}$, and that $\mathscr{V}$ and $\mathscr{V}^{\prime}$ determine respectively $\vdash$ and $\vdash^{\prime}$. We show that if $\mathscr{V} \neq \mathscr{V}^{\prime}$, then $\vdash \neq \vdash^{\prime}$. Without loss of generality, we can take the supposition that $\mathscr{V} \neq \mathscr{V}^{\prime}$ as implying the existence of $v \in \mathscr{V}, v \notin \mathscr{V}^{\prime}$. Since $\mathscr{V}$ determines $\vdash, T(v) \not \nvdash U \backslash T(v)$. But $v$ is the only valuation (for $U$ ) which assigns $\mathrm{T}$ to every element of $T(v)$ and $\mathrm{F}$ to every other element of $U$, so since $v \notin \mathscr{V}^{\prime}, T(v) \vdash^{\prime} U \backslash T(v)$. Thus $\vdash \neq \vdash^{\prime}$. As to the latter point, we recall

Theorem 0.2.2. Any Lindenbaum closure relation is determined by the class of characteristic functions of its maximal avoiders.

We can relate Thm. 0.2.2 to the part of Thm. 0.2.1 dealing with closure relations by adopting a uniform notation, with $v_{X}$ for the characteristic function of a set $X$; in other words, $v_{X}$ is the unique valuation satisfying $T\left(v_{X}\right)=X$. The valuations consistent with a closure relation $\vdash$ (those comprising $\mathscr{V}_{a}(\vdash)$ ) are then precisely those of the form $v_{X}$ for $\vdash$-closed $X$, while those invoked in Thm. 0.2 .2 are a proper subset of these. containing only those $v_{X}$ for which $X$ is a maximal avoider; throwing out the other valuations in $\mathscr{V}_{a t}(\vdash)$ makes no difference to the (Lindenbaum) closure relation determined because we leave behind enough to be able to find a valuation verifying all of $X$ but not $u$ whenever $X \not$ $u$. The above points, as well as that below concerning what we call 'conjunctive combinations' of valuations, go back to Carnap [3], esp. \$\$15, 16, 31, 32. (For most purposes, the 'supervaluations' of [6] , Chapter III, $\S 6$, behave like these conjunctive combinations of valuations. Closely related Carnapian themes are developed in $[1],[8],[9]$.

The utility of Thm. 0.2 .2 for logical studies is illustrated especially by the situation that one is faced with in trying to show a certain syntactically characterized consequence relation $\vdash$ to be determined by the class of all boolean valuations. (More precisely, by the class of all \#-boolean valuations for one or more connectives \# of the language concerned: this notion will be defined shortly. The 
idea is that considering only such valuations is a convenient way of associating

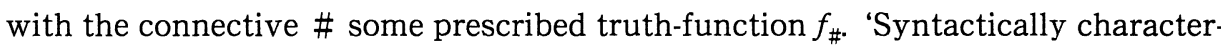
ized' may be taken to mean: specified by means of what, in 0.4 , we shall call generalized rule-like conditions. By a boolean connective we mean simply a connective \# for which the notion of a \#-boolean valuation is defined.) The claim of determination would in this context usually be put in terms of soundness (meaning that $\vdash$ is included in the conseqence relation determined by the class of valuations in question) and completeness (meaning thereby that the converse inclusion holds). The trouble is that just using the characteristic functions of $\vdash$ -closed sets will not yield appropriately boolean valuations, so that Thm. 0.2 .1 is no help, while the characteristic functions of maximal avoiders will deliver such valuations. Indeed in the case of classical sentential logic, for which case we want \#-boolean valuations for every connective \# in the language, the maximal avoiders have a further property, often called 'maximal consistency'. To avoid a bias in favour of such specifically 'logical' applications, however, will speak of absoluteness in this connexion, calling a maximal avoider $W \subseteq U$ an absolute maximal avoider when $W$ maximally avoids every $x \in U \backslash W$. Note that when $\vdash$ is such that every maximal avoider relative to $\vdash$ is absolute, no maximal avoider is properly included in any other maximal avoider. (Examples of $\vdash$ with this property are provided by Prop. 0.6.3; see also the remark following Lemma 2.4.3.) The definition of absoluteness just given renders equivalent the claim that $W$ is an absolute maximal avoider (relative to the closure relation $\vdash$ ) and the claim that $W \neq U$ and $C_{\vdash}(W \cup\{x\})=U$ for all $x \notin W$.

We turn from the closure operation $T \circ V$ to the Galois-dual closure operation $V \circ T$ (for a given $U$ and $\mathscr{V}$ ). Denoting the associated closure relation by $\Vdash$, we have $\mathscr{V}_{0} \Vdash v$ (for $\mathscr{V}_{0} \subseteq \mathscr{V}, v \in \mathscr{V}$ ) just when for every $u \in U$, if all valuations in $\mathscr{V}_{0}$ assign the value $\mathrm{T}$ to $u$, then $v$ assigns $\mathrm{T}$ to $u$. In some interesting cases $\mathscr{V}$ will be closed under an operation which is Galois-dual to the infinitary conjunction operation $\Lambda$ considered in Example 0.1.1, in the sense of satisfying conditions like (1) and (2) of that Example, but with $\vdash$ replaced by $\Vdash$. (Further elaboration follows shortly.) This operation, which we write as ' $\Delta$ ', takes a class $\mathscr{V}_{0} \subseteq \mathscr{V}$ of valuations to a single valuation $\triangle \mathscr{V}_{0}$ called their (general) conjunctive combination, satisfying, for all $\mathscr{V} \subseteq \mathscr{V}$ :
$\left(1^{\prime}\right) \mathscr{V}_{0} \Vdash \Delta \mathscr{V}_{0}$
$\left(2^{\prime}\right) \Delta \mathscr{V}_{0} \Vdash v$ for all $v \in \mathscr{V}_{0}$.

Unlike the case of Example 0.1.1, we impose here no cardinality constraints: $\mathscr{V}$ is not required to be countable, and $\mathscr{V}_{0}$ is allowed to be empty. The upshot of $\left(1^{\prime}\right)$ and $\left(2^{\prime}\right)$ is that the conjunctive combination of a class of valuations for $U$ is the unique valuation which verifies precisely those elements of $U$ which are verified by each valuation in the class, and the main interest of this notion is given by its role in 
Proposition 0.2.3. For any closure relation $\vdash$ and any $\mathscr{V} \subseteq \mathscr{V}$ al $(\vdash), \Delta \mathscr{V} \in$ $\mathscr{V}_{a t}(\vdash)$.

Since $T(\Delta \mathscr{V})$ is $\cap\{T(v)\}_{v \in \mathscr{r}}$, Prop. 0.2.3, which will be of considerable use to us in $\$ \S 1,2$, is a special case of the fact that the intersection of any family of closed (here: $\vdash$-closed) sets is closed. Some special cases are worth noting separately. Given $U$, the unique valuation (for $U$ ) which assigns $T$ to every element of $U$ will be denoted by $v_{\mathrm{T}}$. (We suppress mention of $U$ in this notation.) Further, when $\mathscr{V}=\left\{v_{1}, v_{2}\right\}$, we write $v_{1} \Delta v_{2}$ for $\Delta \mathscr{V}$. Part (i) of the following is then a special case of 0.2 .3 , while part (ii) reflects the fact that $\Delta \varnothing=v_{\mathrm{T}}$. Part (iii) follows from part (ii) via the general observation that if a closure relation $\vdash$ is determined by a class $\mathscr{V}$ of valuations, then not only is $\mathscr{V}$ a subset of $\mathscr{V}_{a t}(\vdash)$, for any other subset $\mathscr{V}^{\prime}$ of $\mathscr{V}_{a t}(\vdash), \vdash$ is determined by $\mathscr{V} \cup \mathscr{V}^{\prime}$. (In the particular case at issue, $\mathscr{V}^{\prime}=\left\{v_{\mathrm{T}}\right\}$.)

Corollary 0.2.4. For any closure relation $\vdash$ :

(i) if $v_{1}, v_{2} \in \mathscr{T}_{a l}(-)$ then $v_{1} \triangle v_{2} \in \mathscr{V}_{a t}(\vdash)$

(ii) $v_{\mathrm{T}} \in \mathscr{V}_{\text {at }}(\vdash)$.

(iii) Given any class of valuations $\mathscr{V}$ determining $\vdash, \mathscr{V} \cup\left\{v_{\mathrm{T}}\right\}$ also determines $\vdash$.

Though we shall not need this for the sequel, we pause to note a simple relationship obtaining between classes of valuations determining a closure relation $\vdash$, and the class of all valuations consistent with $\vdash$. Given a class of $U$-valuations $\mathscr{V}$, we define: $\mathscr{V}^{\Delta}=\left\{\Delta_{0} \mid \mathscr{V}_{0} \subseteq \mathscr{V}\right\}$. Note that ()$^{\Delta}$ is a closure operation on the power set of the set of all $U$-valuations; we make use of this fact without explicit comment in the following proof.

Theorem 0.2.5. For any closure relation $\vdash$ on a set $U$ and any set $\mathscr{V}$ of $U$ valuations: $\vdash$ is determined by $\mathscr{V}$ iff $\mathscr{V}^{\Delta}=\mathscr{V}_{a t}(\vdash)$.

Proof. 'If': Suppose $\mathscr{V}^{\Delta}=\mathscr{V}_{a l}(\vdash)$, with a view to showing that $\mathscr{V}$ determines $\vdash$. We must show first that for all $X \subseteq U, y \in U$, if $X \vdash y$ and $v \in \mathscr{V}$, then $v(x)=\mathrm{T}$ for each $x \in X$, implies $v(y)=\mathrm{T}$, i.e., that $v \in \mathscr{V}_{a t}(\vdash)$. But since $\mathscr{V} \subseteq$ $\mathscr{V}^{\Delta}=\mathscr{V}_{a}(\vdash)$, this is immediate. Conversely, suppose $X \nvdash y$. We need $v \in \mathscr{V}$ with $v(x)=\mathrm{T}$ for each $x \in X$, but $v(y)=\mathrm{F}$. Now we have a valuation $v^{\prime} \in \mathscr{V}_{a t}(\vdash)$ answering to this description, so since $\mathscr{V}^{\Delta}=\mathscr{V}_{a t}(\vdash), v^{\prime}=\Delta \mathscr{V}_{0}$ for some $\mathscr{V}_{0} \subseteq \mathscr{V}$. As $v^{\prime}(y)=\mathrm{F}$, there is some $v^{\prime \prime} \in \mathscr{V}_{0}$ with $v^{\prime \prime}(y)=\mathrm{F}$. Since $v^{\prime}$ verifies all elements of $X$, this is the case for every valuation in $\mathscr{V}_{0}$, and hence in particular for $v^{\prime \prime}$. Thus we may take $v^{\prime \prime}$ as the desired $v \in \mathscr{V}$.

'Only if': Suppose $\vdash$ is determined by $\mathscr{V}$. First we show $\mathscr{V}^{\Delta} \subseteq \mathscr{V} a t(\vdash)$. Since $\mathscr{V}$ $\subseteq \mathscr{V}_{\text {at }}(\vdash)$, we have $\mathscr{V}^{\Delta} \subseteq \mathscr{V}_{a t}(\vdash)^{\Delta}$. But $\mathscr{V}_{a t}(\vdash)^{\Delta} \subseteq \mathscr{V}_{a t}(\vdash)$ by Prop. 0.2.3. Therefore $\mathscr{V}^{\Delta} \subseteq \mathscr{V}_{a t}(\vdash)$. 
Turning to the converse inclusion, $\mathscr{V}_{a t}(\vdash) \subseteq \mathscr{V}^{\Delta}$, take $v \in \mathscr{V}_{\text {al }}(\vdash)$. We must find $\mathscr{V}_{0} \subseteq \mathscr{V}$ with $v=\Delta \mathscr{V}_{0}$. Now $v$ is the characteristic function of some $\vdash$-closed set $X$, and we now use the notation introduced after Thm. 0.2 .2 , writing $v_{X}$ for $v$, noting that

$$
v_{X}=\Delta\left\{v_{Y} \in \mathscr{V}_{a t}(\vdash) \mid Y \supseteq X\right\} .
$$

Thus the desired $\mathscr{V}_{0}$ is $\left\{v_{Y} \in \mathscr{V}_{a t}(\vdash) \mid Y \supseteq X\right\}$ : the set of $v^{\prime}$ consistent with $\vdash$ such that $T\left(v^{\prime}\right) \supseteq T(v)$.

Returning to Coro. 0.2 .4 , we observe that the operation $\triangle$, of binary conjunctive combination, on valuations which figures in part (i) thereof, illustrates a general phenomenon. Let us say that a Galois connexion between $L$ and $R$, whose induced relation $\subseteq L \times R$ is $\varphi$, has (binary) conjunctive combinations on the left if for every $l_{1}, l_{2} \in L$ there is some $l_{3} \in L$ such that for all $r \in R: \varphi\left(l_{3}, r\right)$ if and only if $\varphi\left(l_{1}, r\right)$ and $\varphi\left(l_{2}, r\right)$; in this case we call $l_{3}$ a conjunctive combination of $l_{1}$ and $l_{2}$. The Galois connexion has (binary) conjunctive combinations on the right if for every $r_{1}, r_{2} \in R$ there is some $r_{3} \in R$ such that for all $l \in L: \varphi\left(l, r_{3}\right)$ if and only if $\varphi\left(l, r_{1}\right)$ and $\varphi\left(l, r_{2}\right)$; such an $r_{3}$ is a conjunctive combination of $r_{1}$ and $r_{2}$. These two notions are Galois-dual to each other in that they result from interchanging references to left and right elements and replacing $\varphi$ by its converse. In the Galois connexions of interest to us here, between some set $U$ and some set $\mathscr{V}$ of valuations for $U$, with $\varphi(u, v)$ when $v(u)=\mathrm{T}$, we may or may not have conjunctive combinations on the right, though if $\mathscr{V}$ is the set of all valuations for $U$, we clearly do, as indeed we also do (by 0.2 .4 (ii)) when $\mathscr{V}$ is $\mathscr{V}_{a t}(\vdash)$ for any closure relation $\vdash$ on $U$. But if $v_{1}$ and $v_{2}$ have a conjunctive combination in $\mathscr{V}$, it is unique, since valuations assingning $\mathrm{T}$ to the precisely the same elements of $U$ are identical, justifying the functional notation $v_{1} \triangle v_{2}$ for this combination. Conjunctive combinations on the left arise most familiarly when $U$ is a set of formulas closed under the binary operation (connective) of conjunction $\wedge$, and the valuations in $\mathscr{V}$ are what we shall call $\wedge$-boolean, meaning by this valuations $v$ such that for all, $u_{1}, u_{2} \in U: v\left(u_{1} \wedge u_{2}\right)=\mathrm{T}$ if and only if $v\left(u_{1}\right)=\mathrm{T}$ and $v\left(u_{2}\right)=\mathrm{T}$. In this case, only a weaker justification is available for the use of the functional notation, namely, that any for any $u, u^{\prime}$ both satisfying to the above condition on $u_{1} \wedge u_{2}$, we have $u \vdash u^{\prime}$ and $u^{\prime} \vdash u$ where $\vdash$ is the closure relation (consequence relation) determined by the class of $\wedge$-boolean valuations (for $U$, assumed closed under $\wedge$ ). A more extensive discussion of uniqueness for connectives may be found in [5].

The Galois duality between $\wedge$ and $\triangle$ can be succinctly exhibited in terms of our $(V, T)$ Galois connexions between $U$ and $\mathscr{V}$ by noting that just as when $\mathscr{V}$ contains only $\wedge$-boolean valuations, we have $V(x \wedge y)=V(x) \cap V(y)$, for all $x, y \in U$, so we have $T\left(v_{1} \triangle v_{2}\right)=T\left(v_{1}\right) \cap T\left(v_{2}\right)$, for all $v_{1}, v_{2} \in \mathscr{V}$, whenever the left-hand side is defined. (As already remarked, it is defined in the case in which $\mathscr{V}$ comprises all $U$-valuations, as well as in numerous other cases of interest: see 
Coro. 0.2.4 (i) and Prop. 0.3.2 (i).)

The above evidently represents a special case of the more general phenomenon of Galois duality between sentence connectives and operations on valuations, further aspects of which will be our focus of concern in the following sections. To capture the usual truth-table constraints on how valuations are to treat compound formulas, we list a few further cases. The connectives we have in mind are (inclusive) disjunction ( $\vee$ : binary), implication $(\rightarrow$ : binary), (material) equivalence ( $\leftrightarrow$ : binary), exclusive disjunction ( $\vee$ : binary), negation ( $\neg$ : singulary), the truth and falsity constants ( $T$ and $\perp$ : zeroary). A valuation $v$ for $U$ assumed closed under the connective in question is $\vee$-boolean when for all $u_{1}, u_{2} \in U$, $v\left(u_{1} \vee u_{2}\right)=\mathrm{T}$ iff $v\left(u_{1}\right)=\mathrm{T}$ or $v\left(u_{2}\right)=\mathrm{T}$ (or both), $\rightarrow$-boolean when for all $u_{1}, u_{2}, v\left(u_{1} \rightarrow u_{2}\right)=\mathrm{T}$ iff $v\left(u_{1}\right)=\mathrm{F}$ or $v\left(u_{2}\right)=\mathrm{T}$ (or both), $\leftrightarrow$-boolean when for all $u_{1}, u_{2}, v\left(u_{1} \leftrightarrow u_{2}\right)=\mathrm{T}$ iff $v\left(u_{1}\right)=v\left(u_{2}\right)=\mathrm{T} ; \vee$-boolean when for all $u_{1}, u_{2}$, $v\left(u_{1} \underline{\vee} u_{2}\right)=\mathrm{T}$ iff $v\left(u_{1}\right) \neq v\left(u_{2}\right) ; \neg$-boolean when for all $u, v(\neg u)=\mathrm{T}$ iff $v(u)=\mathrm{F}, \mathrm{T}$-boolean $v(\mathrm{~T})=\mathrm{T}$; and $\perp$-boolean when $v(\perp)=\mathrm{F}$. The operations on classes of valuations which are Galois-dual to these connectives, as $\Delta$ is to $\wedge$, need to be baptized. That corresponding to $T$ has already been seen (Coro. 0.2.4 (ii)): $v_{\mathrm{T}}$. Just as $T$ is verified by every appropriately boolean valuation, so $v_{\mathrm{T}}$ is a valuation verifying every element of $U$. (We are treating a 0 -place opration on $\mathscr{V}$ as a distinguished element of $\mathscr{V}$.) Similarly corresponding to $\perp$ we have the valuation $v_{F}$ which falsifies every element of $U$. (Note that neither of these valuations is \#-boolean for all of the above connectives \#; for example, neither is $\neg$-boolean.) In the same vein, we describe the operation Galois-dual to $\vee$ as forming disjunctive combinations on the right, and denote it by $\nabla: v_{1} \nabla v_{2}$ is that valuation assigning $\mathrm{T}$ to precisely those elements of $U$ to which either $v_{1}$ or $v_{2}$ (or both) assigns $T$. The words 'on the right' will be omitted when we return to examine this case again in $\S 2$, though of course by analogy with what was said about conjunctive combinations on the left and right, we can make sense of the idea of disjunctive combinations on the left, and when $U$ is a set of formulas closed under $\vee$ and $\mathscr{V}$ a set of $\vee$-boolean valuations, the disjunctive combinaton of two elements on the left is simply (to within equivalence) their disjunction. Similar remarks apply in the case of the remaining operations, for which we shall simply introduce terminology and notation. (Note that we shall use only for binary disjunctive combination, and not for the general version poset-dual to the opera-

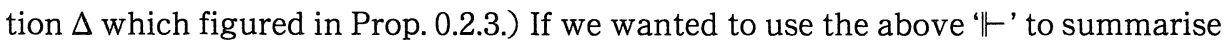
the definition of $\nabla$, we would naturally reconstrue it as standing for a generalized closure relation rather than a closure relation, so that we could write not only:

$$
v_{1} \Vdash v_{1} \nabla v_{2} \text { and } v_{2} \Vdash v_{1} \nabla v_{2}
$$

but also: 


$$
v_{1} \nabla v_{2} \Vdash v_{1}, v_{2}
$$

where $\mathscr{V}_{0} \Vdash \mathscr{V}_{1}$ means that for all $u \in U$, if $v(u)=\mathrm{T}$ for each $v \in \mathscr{V}_{0}$, then $v^{\prime}(u)=\mathrm{T}$ for some $v^{\prime} \in \mathscr{V}_{1}$. This would be the Galois-dual version of the familiar use of generalized consequence relations in logic for the sake of respecting poset duality in the notation. However, we shall not be making further use of either the closure or the generalized closure construal of ' $\Vdash$ '. (As remarked in the Outline, we are interested in "the effect of such assumptions as $(\dagger \dagger)$ on the consequence relation $\vdash$ (on $U$ ) determined by $\mathscr{V}^{\prime \prime}$; $(\dagger \dagger)$ was what we would now call the assumption that $\mathscr{V}$ is closed under disjunctive combination of valuations. The point is to explore the effects of such assumptions on closure and generalized closure relations on $U$, rather than, as with $\Vdash$, on $\mathscr{V}$.)

Before proceeding with the remaining connectives listed above, we pause to note a simple characterization of the Galois-dual of a given connective \# for which the notion of a \#-boolean valuation is taken to have been defined. Suppose \# is $n$-ary. Then there is associated with \#, on all such valuations, an $n$-ary truthfunction $f_{\#}:\{\mathrm{T}, \mathrm{F}\}^{n} \rightarrow\{\mathrm{T}, \mathrm{F}\}$, the details of which association may be found in the discussion between (8) and (9) of 0.3 . The Galois-dual \# of \# is then that $n$-ary operation on valuations satisfying, for all $v_{1}, \cdots, v_{n}$ : \# $\left(v_{1}, \cdots, v_{n}\right)(u)=$ $f_{\#}\left(v_{1}(u), \cdots, v_{n}(u)\right)$ for every $u \in U$.

Turning to the remaining cases, we use the following notation. The operation Galois-dual to negation $(\neg)$ is the one-place operation we shall indicate by overlining: - Thus $\bar{v}$ is the valuation assiging $\mathrm{T}$ to precisely those elements of $U$ mapped by $v$ to $\mathrm{F}$ (and, as goes without saying since our valuations are bivalent, vice versa). This is a special case of the general phenomenon of what we call negative objects on the right: elements $\bar{r} \in R$ such that for every $l \in L \varphi(l, \bar{r})$ just in case not $\varphi(l, r)$, where $\varphi$ is the induced binary relation of the Galois connexion in question between $L$ and $R$. Galois-dual to $\underline{V}$, we have $\underline{\nabla}$ : exclusive disjunctive combination ('on the right'), to $\rightarrow$, we have $\Rightarrow$, forming implicative combinations, and to $\leftrightarrow$ we have what we shall write as $\sim$. We call this 'equivalential combination' $v_{1} \sim v_{2}$ of valuations $v_{1}, v_{2}$, the match of $v_{1}$ with $v_{2}$, and refer to $\sim$ as the matching operation, in the interests of brevity. We will motivate the study of closure and generalized closure operations determined by classes of valuations closed under matching in $\S 1$. (See the Outline for a more general motivation.) Having made a start on that study in $\S 1$, we return to it in $\S 3$, after looking in $\S 2$ at analogous issues concerning the other operations Galois-dual to the connectives listed above. (In 2.5 certain further boolean connectives will be introduced, along with their Galois duals. We defer their baptism until then to avoid notational overloat at this point.) 


\subsection{Combinations on Both Sides of a Galois Connexion}

The above discussion of operations Galois-dual to the usual boolean connectives is not intended to suggest that we will be concerned with conjunctive, disjunctive, etc., modes of combination on both the left and the right sides of the Galois connexions (between $U$ and $\mathscr{V}$ ) in which we are taking a special interest. We shall suppose, in $\S \S 1-3$, only that the right hand side $(\mathscr{V})$ is closed under the operations in question. In fact, making such suppositions for both sides at once severely constrains the behaviour of the induced relation $\varphi$. We shall illustrate this in Prop. 0.3.1, before passing on (Prop. 0.3.2) to cases in which these constraints do not arise.

Proposition 0.3.1. Let $\varphi$ be the relation induced by a Galois connexion between sets $L$ and $R$. Then

(i) if the Galois connexion has conjunctive combinations on the left and disjunctive combinations on the right, or vice versa, then $\varphi$ satisfies, for all $r_{1}, r_{2} \in R$, $l_{1}, l_{2} \in L$, the condition:

$$
\varphi\left(l_{1}, r_{1}\right) \text { and } \varphi\left(l_{2}, r_{2}\right) \text { imply } \varphi\left(l_{1}, r_{2}\right) \text { or } \varphi\left(l_{2}, r_{1}\right)
$$

and

(ii) if the Galois ccnnexion has negative objects on the left and either conjunctive or disjunctive combinations on the right, then $\varphi$ must satisfy, for all $l \in L$ :

$$
\varphi(l, r) \text { for some } r \in R \text { implies } \varphi(l, r) \text { for all } r \in R .
$$

Proof. By way of illustration, we give the argument for (ii), in the case that there are negative objects on the left and conjunctive combinations on the right. Suppose, for this case, that the condition cited fails. That is, we have $l \in L$ with $\varphi\left(l, r_{1}\right)$ and not $\varphi\left(l, r_{2}\right)$. Thus for a conjunctive combination $r_{1} \triangle r_{2}$ of $r_{1}$ with $r_{2}$, we have: not $\varphi\left(l, r_{1} \triangle r_{2}\right)$, so for a negative object $\bar{l}$ corresponding to $l$ on the left, $\varphi\left(\bar{l}, r_{1} \triangle r_{2}\right)$. So we have both $\varphi\left(\bar{l}, r_{1}\right)$ and $\varphi\left(\bar{l}, r_{2}\right)$. But the first of these conclusions contradicts the fact that $\varphi\left(l, r_{1}\right)$.

We remark that the condition (on $\varphi$ ) cited in Prop. 0.3.1(i) has been discussed under the lebel 'the Cross-Over Condition' in [13], $\S 3$ of which provides references to its appearance under various other names in diverse literatures.

The condition just mentioned has what is from a logical point of view a very disturbing effect on the closure relations determined by classes of valuations satisfying it (when $\varphi$ is the relation 'is verified by'), namely:

$$
\text { For all } x, y \in U: x \vdash y \text { or } y \vdash x
$$

while the condition cited in 0.3 .1 is similarly pathological (indeed more so, since it implies (3)) from this perspective: 


$$
\text { For all } x, y \in U: \vdash y \text { or } y \vdash x \text {. }
$$

(The lop-sided appearance of (4) is an artefact of casting the discussion in terms of closure relations: for generalized closure relations we can do away with the asymmetry and say that the effect of the condition in question is to secure

$$
\text { For all } y \in U: \vdash y \text { or } y \vdash \text {. }
$$

More explicitly this means: $\varnothing \vdash y$ or else $y \vdash \varnothing$.)

Since some of the conditions we shall be considering below also look somewhat unfamiliar from the vantage point of traditional logical studies, it is worth saying what is so especially 'disturbing' or 'pathological' about (3) and (4), as compared with, say, (5), which will appear as a simplified version of a condition called (Left Unions ${ }^{\exists}$ ) in 2.2, or (6), a special case of what we call $\left(\right.$ Reverse $^{\mathrm{CC}}$ ) in 2.1 , or (7), which is a strengthened version of a condition called (Exchange), introduced in 0.4 below:

$$
\begin{gathered}
X_{0}, X_{1} \vdash y \text { implies } X_{0} \vdash y \text { or } X_{1} \vdash y\left(\text { for all } X_{0}, X_{1} \subseteq U\right) \\
x \vdash y \text { implies } y \vdash x(\text { all } x, y \in U) \\
X, x \vdash y \text { implies } y \vdash x \text { or } X \vdash y(\text { all } X \subseteq U, x, y \in U) .
\end{gathered}
$$

What (5), (6), and (7) have in common which sets them apart - their unfamiliarity notwithstanding-from (3) and (4), is that they are all conditions on closure relations which are satisfied by the smallest closure relation $\vdash_{0}$ on any geiven underlying set $U$. This closure relation is defined by: $X \vdash_{0} x$ if and only if $x \in X$ (so that all sets are $\vdash_{0}$-closed), and we leave the reader to verify that $\vdash_{0}$ has properties (5), (6), (7), while lacking (3) and (4). Every condition we impose on closure relations in $\S 1-\S 3$ in order to obtain a class of closure relations determined by some collection of valuations closed under one of the Galois duals of the boolean sentence connectives listed in 0.2 is a conditon satisfied by $\vdash_{0}$, rather than one of the 'pathological' conditions illustrated above. To see this, note that $\vdash_{0}$, as the least closure relation on some set $U$, is determined by the class of all valuations for $U$, and the latter class is clearly closed under any operation you care to name from $U$-valuations to $U$-valuations. (Analogous remarks apply in the case of conditions on generalized closure relations; in this case $\vdash_{0}$ holds between $X$ and $Y$ just when $X \cap Y \neq \varnothing$.)

Conspicuously absent from the examples in Prop. 0.3.1 are cases in which we have the some mode of combination on both the left and the right, and such cases indeed arise naturally in the study of sentential logic. Let $U$ be the language of the 'pure conjunction' fragment of classical (or indeed intuitionistic) logic and $\mathscr{V}$ be the class of all $\wedge$-boolean valuations. Then the Galois connexion $(V, T)$ between $U$ and $\mathscr{V}$ (as described in 0.2 ) has not only conjunctive combinations on the left (formed by $\wedge$ ), but also conjunctive combinations on the right (to be denoted with 
the aid of $\triangle$ ). That is, $\mathscr{V}$ is closed under $\triangle$. For suppose that $v_{1}, v_{2}$, are $\wedge$-boolean valuations and consider their conjunctive combination $v_{1} \triangle v_{2}$. For all $x, y \in U$ we have $v_{1} \triangle v_{2}(x \wedge y)=\mathrm{T}$ iff $v_{1}(x \wedge y)=\mathrm{T}$ and $v_{2}(x \wedge y)=\mathrm{T}$, by the definition of $\triangle$, which holds in turn iff $v_{1}(x)=\mathrm{T}$ and $v_{1}(y)=\mathrm{T}$ and $v_{2}(x)=\mathrm{T}$ and $v_{2}(y)=\mathrm{T}$, since $v_{1}$ and $v_{2}$ are $\wedge$-boolean. And we may rearrange the conjuncts of this four-termed conjunction to give the equivalent:

$$
v_{1}(x)=\mathrm{T} \text { and } v_{2}(x)=\mathrm{T} \text { and } v_{1}(y)=\mathrm{T} \text { and } v_{2}(y)=\mathrm{T}
$$

which means, again invoking the definition of $\triangle$, that

$$
v_{1} \triangle v_{2}(x)=\mathrm{T} \text { and } v_{1} \triangle v_{2}(y)=\mathrm{T} .
$$

Since $x$ and $y$ were arbitrary elements of $U$, we have shown that $v_{1} \triangle v_{2}$ : is an $\wedge$-boolean valuation.

To see what precisely is being used in the above argument, let us run through it with respect to an arbitrary (but still for the moment) binary connective \# under wihch we assume $U$ is closed and for which we assume a notion of \#boolean valuation is defined using the analogous connective of English, represented by \#: $v$ is \#-boolean iff for all $x, y \in U, v(x \# y)=\mathrm{T}$ just in case $v(x)=\mathrm{T} \# \bar{v}(y)=\mathrm{T}$, and the Galois-dual operation $\widehat{\#}$ on valuations defined by $v_{1} \# v_{2}(u)=\mathrm{T}$ iff $v_{1}(u)=\mathrm{T} \# v_{2}(u)=\mathrm{T}$, for all $v_{1}, v_{2} \in \mathscr{V}, u \in U$. (Alternatively, as foreshadowed in 0.2 , we define $v_{1} \# v_{2}(u)=f_{\#}\left(v_{1}(u), v_{2}(u)\right)$, the precise

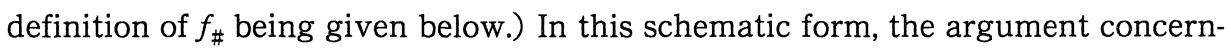
ing conjunction above, appears as follows. (Of course in that particular argument $\#$ is 'and', with \#, $\widehat{\#}$, being respectively $\wedge, \triangle$.)

$$
\begin{aligned}
& v_{1} \# v_{2}(x \# y)=\mathrm{T} \text { iff } v_{1}(x \# y)=\mathrm{T} \# v_{2}(x \# y)=\mathrm{T} \\
& \text { iff }\left(v_{1}(x)=\mathrm{T} \# v_{1}(y)=\mathrm{T}\right) \#\left(v_{2}(x)=\mathrm{T} \# v_{2}(y)=\mathrm{T}\right) \\
& \text { iff }\left(v_{1}(x)=\mathrm{T} \# v_{2}(x)=\mathrm{T}\right) \stackrel{\#}{\underline{\#}}\left(v_{1}(x)=\mathrm{T} \# v_{2}(y)=\mathrm{T}\right) \\
& \text { iff }\left(v_{1} \widehat{\#} v_{2}(x)=\mathrm{T}\right) \#\left(v_{1} \# v_{2}(y)=\mathrm{T}\right) \text {. }
\end{aligned}
$$

The first line here unpacks the definition of $\mathbb{\#}$, while transition to the second line records the \#-boolean nature of the valuations $v_{1}$ and $v_{2}$, and the transition from the third to the final line again exploits the definition of $\#$. The passage from the second to the third line requires a special assumption about the logical behaviour of \#, which is reflected by that of \# when attention is restricted to what we are calling \#-boolean valuations, namely, that for all statements $\alpha, \beta, \gamma, \delta$

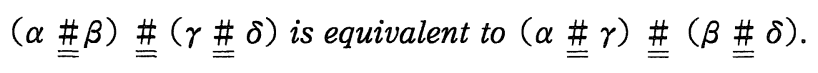

In the case of the argument presented schematically above, $\alpha, \beta, \gamma, \delta$, are respectively the statements: $v_{1}(x)=\mathrm{T}, v_{1}(y)=\mathrm{T}, v_{2}(x)=\mathrm{T}$, and $\left.v_{2}(y)=\mathrm{T}\right)$. The concrete version of the argument, with 'and' for '\#', showing that the conjunctive 
combination of any pair of $\wedge$-boolean valuations is $\wedge$-boolean, is valid because 'and' (taken for '\#') satisfies (8).

We can put this point another way, by looking at the truth-functions involved. Over the class of all \#-boolean valuations (for any \# for which the notion '\#-boolean' has been defined: though for the moment we continue to restrict attention to the case of binary \#), a (two-place) truth-function $f_{\#}$ is associated with \#, namely the unique such function which satisfies, for each such valuation $v$ and every $x, y \in U: v(x \# y)=f_{\#}(v(x), v(y))$. Our special assumption can then be formulated as the condition that this function satisfies what has been called the 'medial law', stated here for readability using '\#' (and infix notation) rather than ' $f_{\#}$ ', and now with $\alpha, \beta, \gamma, \delta$ standing for truth-values $(\mathrm{T}, \mathrm{F})$ rather than statements:

$$
(\alpha \# \beta) \#(\gamma \# \delta)=(\alpha \# \gamma) \#(\beta \# \delta) .
$$

(The name 'medial law' for the identity (9), interpreted in an arbitrary groupoid, and references to further discussions of this identity under various names, may be found in [4], pp.60,68.) Of the boolean connectives explicitly listed in 0.2 , not only $\wedge$ (more accurately: $f_{\wedge}$ ), but also $\vee, \leftrightarrow$, and $\underline{\vee}$, satisfy (9), and we therefore have, (i) - (iv) of the following:

Proposition 0.3.2. (i) The class of all $\wedge$-boolean valuations is closed under $\triangle$. (ii) The class of all $\vee$-boolean valuations is closed under $\nabla$.

(iii) The class of all $\leftrightarrow$-boolean valuations is closed under .

(iv) The class of all $\underline{\vee}$-boolean valuations is closed under $\underline{\nabla}$.

(v) The class of all $\neg$-boolean valuations is closed under ${ }^{-}$.

Part (v) concerns a singulary rather than a binary truth-function, so its content does not fall immediately out from the above discussion, though it will emerge as a consequence of suitably generalizing that discussion in due course. (See the final sentence of the present subsection; alternatively, the reader may easily make an ad hoc verification now.) Several of the parts of Prop. 0.3.2 will be of use to us below, beginning with an appeal to part (iii) in Example 1.2.5. Of course, the above formulation is a little casual in the following respect. We should strictly say, for 0.3 .2 (i), for example: for any $U$ closed under $\wedge$, the class of all $\wedge$-boolean valuations for $U$ is closed under $\triangle$; similarly in the other cases.

The binary truth-functions figuring in Prop. 0.3.2 (i) - (iv) conspicuously satisfy not only the medial law (9) but are also commutative and associative, which we now proceed to show (Thm. 0.3.5) is no coincidence, even though neither commutativity nor associativity is a consequence (in the sense of equational logic) of 'mediality'. They are both consequences of the medial law together with the additional assumptions that we are considering only functions on a two-element set and that the function concerned depends on both of its arguments ("has no 
inessential variables", as it is sometimes put) ; to express the latter property we describe the functions concerned as essentially binary. We put these points by taking our set to be $\{\mathrm{T}, \mathrm{F}\}$, so that the discussion speaks (without any loss of generality) of binary truth-functions. Since there are only 16 such functions, we could draw our conclusions from an exhaustive examination of all the cases. But this would not be very informative. The approach below seems more explanatory, segregating out, in Lemmas 0.3.3, 0.3.4, two things which do depend on the additional assumptions just described-commutativity and the existence of (two-sided) identity elements - from something which does not: namely that any binary operation satisfying the medial law and possessing the latter two properties must be associative (as we observe in the proof of Thm. 0.3.5).

The following notation will be helpful: given $x \in\{\mathrm{T}, \mathrm{F}\}, \bar{x}$ is whichever element of $\{\mathrm{T}, \mathrm{F}\} x$ isn't; we also write $x \cdot y$ as $x y$ when convenient.

Lemma 0.3.3. Any essentially binary truth-function satisfying the medial law is commutative.

Proof. Assume $\cdot$ is a binary truth-function which satisfies the medial law and depends on both its arguments. Since - depends on its first argument, there is $x \in\{\mathrm{T}, \mathrm{F}\}$ with $x \mathrm{~T} \neq x \mathrm{~F}$, and since $\cdot$ depends on its second argument we also have $y \in\{\mathrm{T}, \mathrm{F}\}$ with $\mathrm{T} y \neq \mathrm{F} y$. There are thus four possibilities, in the first two of which we do not even need the medial law to establish commutativity:

Case $1: x=y=\mathrm{T}$. So TT $\neq \mathrm{TF}$ and TT $\neq \mathrm{FT}$. Thus $\mathrm{TF}=\mathrm{FT}$, and $\cdot$ is accordingly commutative.

Case 2: $x=y=\mathrm{F}$. So $\mathrm{FT} \neq \mathrm{FF}$ and $\mathrm{TF} \neq \mathrm{FF}$. This again $\mathrm{TF}=\mathrm{FT}$.

$\overline{\text { Case }}$ 3: $x=\mathrm{T}, y=\mathrm{F}$. So TT $\neq \mathrm{TF}:$ and $\mathrm{TF} \neq \mathrm{FF}$. Therefore $\mathrm{TT} \neq \mathrm{FF}$.

Now suppose that $\cdot$ is not commutative, so that $\mathrm{TF} \neq \mathrm{FT}$. Then we must FT $=\mathrm{FF}$ $=\mathrm{FT}=\alpha$ (say), with $\mathrm{TF}=\bar{\alpha}$. The medial law gives $(\mathrm{TT})(\mathrm{FT})=(\mathrm{TF})(\mathrm{TT})$, which is to say $\alpha \alpha=\bar{\alpha} \alpha$; similarly we have (FF)(TF) $=(\mathrm{FT})$ (FT), so $\alpha \bar{\alpha}=\alpha \alpha$. Therefore $\alpha \bar{\alpha}=\bar{\alpha} \alpha$, and $\cdot$ must be commutative after all.

Case 4: Similar to Case 3.

Lemma 0.3.4. For any essentially binary truth-function satisfying the medial law there is an identity element.

Proof. Suppose for - a function as described neither $\mathrm{T}$ nor $\mathrm{F}$ is an identity element (neutral element). Since by Lemma $0.3 .3, \cdot$ is commutative, we do not need to distinguish left-identities from right-identities, though we work here with a formulation denying, specifically, the existence of identity elements on the left: there are $x, y \in\{\mathrm{T}, \mathrm{F}\}$ with $\mathrm{T} x \neq x$ and $\mathrm{F} y \neq y$; thus $\mathrm{T} x=\bar{x}$ and $\mathrm{F} y=\bar{y}$, giving four cases:

Case 1: $x=y=\mathrm{T}$. So $\mathrm{TT}=\mathrm{F}$ and $\mathrm{FT}=\mathrm{F}$. Since by commutativity we also have 
$\mathrm{TF}=\mathrm{F}$, to avoid - 's being a constant function (depending on neither argument), we must have $F F=T$. The medial law gives $(\mathrm{TT})(\mathrm{FF})=(\mathrm{TF})(\mathrm{TF})$, whose left-hand side evaluates to $\mathrm{F} \cdot \mathrm{T}=\mathrm{F}$, but whose right-hand side evaluates to $\mathrm{F} \cdot \mathrm{F}$ $=\mathrm{T}$. Thus Case 1 cannot arise.

Case 2: $x=\mathrm{T}, y=\mathrm{F}$. So TT $=\mathrm{F}$ and $\mathrm{FF}=\mathrm{T}$. The medial law gives (TT)(FF) $=(\mathrm{TF})(\mathrm{TF})$, and thus, inserting the given values of $\mathrm{TT}$ and $\mathrm{FF}: \mathrm{FT}=(\mathrm{TF})(\mathrm{TF})$, and so by commutativity, $\mathrm{TF}=(\mathrm{TF})(\mathrm{TF})$; but if $\mathrm{TF}=\mathrm{T}$ this contradicts the fact that $\mathrm{TT}=\mathrm{F}$ : and if $\mathrm{TF}=\mathrm{F}$, it contradicts the fact that $\mathrm{FF}=\mathrm{T}$.

Case 3: $x=\mathrm{F}, y=\mathrm{T}$. This time, substituting into $\mathrm{T} x=\bar{x}$ and $\mathrm{F} y=\bar{y}$ : gives $\mathrm{TF}$ $=\mathrm{T}$ and $\mathrm{FT}=\mathrm{F}$, immediately contradicting commutativity.

Case 4: $x=\mathrm{F}, y=\mathrm{F}$. So $\mathrm{TF}=\mathrm{T}$ and $\mathrm{FF}=\mathrm{T}$, and an argument as for Case 1 delivers a contradiction.

Theorem 0.3.5. An essentially binary truth-function satisfies the medial law if and only if it is both commutative and associative.

Proof. 'If': Since the medial law is an obvious equational consequence of the commutative and associative laws taken together, the 'if' direction holds for any binary operation whatever.

'Only if. Commutativity was established in Lemma 0.3.3. For associativity, we use Lemma 0.3.4, which promised, for a given - satisfying the conditions of the Theorem, an identity element $e$. Then the reasoning is $x(y z)=(x e)(y z)=$ $(x y)(e z)=(x y) z$.

We pause to observe, though we shall not need to appeal to it in what follows, that the generalization of (9) appropriate for securing the same effect (viz: analogues of Prop. 0.3.2 for truth-functions of arbitrary arity) is (10), which works to give the needed (11); here \# (and therefore \# and \#) is a $k$-ary truth-function and $\alpha_{\jmath}^{2} \in\{\mathrm{T}, \mathrm{F}\}$ :

$$
\begin{aligned}
& \#\left(\left(\#\left(\alpha_{1}^{1}, \alpha_{2}^{1}, \cdots, \alpha_{k}^{1}\right), \#\left(\alpha_{1}^{2}, \alpha_{2}^{2}, \cdots, \alpha_{k}^{2}\right), \cdots, \#\left(\alpha_{1}^{k}, \alpha_{2}^{k}, \cdots, \alpha_{k}^{k}\right)\right)=\right. \\
& \#\left(\#\left(\alpha_{1}^{1}, \alpha_{1}^{2}, \cdots, \alpha_{1}^{k}\right), \#\left(\alpha_{2}^{1}, \alpha_{2}^{2}, \cdots, \alpha_{2}^{k}\right), \cdots, \#\left(\alpha_{k}^{1}, \alpha_{k}^{2}, \cdots, \alpha_{k}^{k}\right)\right) .
\end{aligned}
$$

(11) For arbitrary $x_{1}, \cdots, x_{k} \in U, v_{1}, \cdots, v_{k} \in \mathscr{V}$, the claims that

$$
\begin{aligned}
& \#\left[v_{1}\left(\#\left(x_{1}, \cdots, x_{k}\right)\right)=\mathrm{T}, v_{2}\left(\#\left(x_{1}, \cdots, x_{k}\right)\right)=\mathrm{T}, \cdots, v_{k}\left(\#\left(x_{1}, \cdots, x_{k}\right)\right)=\mathrm{T}\right] \\
& \text { and } \\
& \#\left[\begin{array}{l}
\# \\
\text { are equivalent. }
\end{array}\right.
\end{aligned}
$$

It is worth noting that for the case of $k=1,(10)$ and (11) are automatically satisfied, (10), for example, reducing to the assertion that $\# \# \alpha=\# \# \alpha$, under 
which observation we may subsume the fact that the class of all $\neg$-boolean valuations is closed under formation of negative objects (= Prop. 0.3.2 (v)).

\subsection{The Exchange Property}

A closure operation on a set $U$ is said to have the Exchange Property if the associated closure relation - which in that case we shall also describe as having the Exchange Property - satisfied the conditon:

(Exchange) For all $X \subseteq U, x, y \in U: X, x \vdash y$ implies $X, y \vdash x$ or $X \vdash y$.

This condition has been considered in the literature on (in)dependence in various areas, and in particular in connexion with linear independence in vector spaces. Here the closure of a set of vectors (over a given field) comprises those obtainable from the given set by taking 'linear combinations' and the fact that this (finitary) closure operation enjoys the Exchange Property is used to derive the conclusion that irredundant bases for a given closed set all have the same cardinality. Extensive references to the relevant literature may be found on $\mathrm{pp}$. 129,132 , of the survey [11]. (With some closure operation $C$ in mind, $X$ is a basis for $Y$ if $C(X)=Y$; such an $X$ is irredundant if in addition for any proper subset $X_{0}$ of $X, C\left(X_{0}\right)$ is a proper subset of $C(X)=Y$. Often in the linear algebra literature, what has just been defined as a basis is called instead a spanning set, with 'basis' reserved to mean 'irredundant basis'. In the logical tradition, with $C$ a consequence operation, an irredundant basis for $Y$ may be called an independent axiomatization of $Y$; see [29] for a full discussion.)

Aside from the fact that it will come to our attention below (in $\$ \S 1,3$ ), we mention (Exchange) here for two illustrative purposes. (In 0.6 we will make a few observations of a more technical nature on this condition.) The first is over the utility of thinking in terms of closure relations rather than specifically consequence relations, understood as closure relations on the set of formulas of some language. Whether a language is understood in concrete terms as a set of strings of symbols (with sentential connectives serving to make longer strings from shorter ones), or more abstractly, as any absolutely free algebra (with the connectives as fundamental operations), this is too restrictive to cover the use of ' $\vdash$ ' in (Exchange) for its application to the case of vector spaces, where the set $U$ consists of vectors. In the case which will be of special interest to us in $1.1, U$ consists instead of properties which individuals may have or lack: not as linguistic entities of any sort (least of all formulas of some language). It is for this reason that we avoided using such variables as ' $\mathrm{A}$ ', ' $\mathrm{B}$ ', ' $\mathrm{C}$ ', $\cdots$ or ' $\varphi$ ', ' $\phi$ ', ' $\chi$ ' $\cdots$, over elements of $U$, as is more customary, when stating $(\mathbb{R}),(\mathbb{M})$ and $(\mathbb{T})$ above, not wanting to suggest a specifically linguistic interpretation.

The second feature illustrated by (Exchange) is its disjunctive consequent: either $X, y \vdash u$ or $X \vdash y$. Call a condition on closure or generalized closure 
relations $\vdash$ (with underlying set $U$ ) rule-like if it has the form

$$
\left(\mathscr{A}_{1} \text { and } \cdots \text { and } \mathscr{A}_{m}\right) \text { implies }\left(\mathscr{B}_{1} \text { or } \cdots \text { or } \mathscr{B}_{n}\right)
$$

preceded by a string of universal quantifiers ranging over subsets and elements of $U$, where each $\mathscr{A}_{i}, \mathscr{B}_{j}$ is a $\vdash$-statement, and $n=1$. For a generalized rule-like condition, we drop the restriction on $n$. When $m=0$ we identify (12) with its consequent, and when (with the restriction on $n$ dropped) $n=0$, with the negation of its antecedent. (If $m=n=0$, (12) is interpreted as an inconsistent condition.) Rule-like conditions are so called because the formalization of an area of logic in terms of 'sequents' employs sequent-to-sequent rules which amount to such conditions on the consequence relation or generalized consequence relation associated in the obvious way with the proof-system in question. (If the sequents, as in the familiar natural deduction systems for, e.g., classical or intuitionistic logic, or in a minor recasting of Gentzen's sequent calculus for intuitionistic logic, have exactly one formula on the right, we have a consequence relation, while if either no, or more than one, formula is also allowed on the right, we have a generalized consequence relation. Indeed, a sequent provable in a proof-theoretic presentation of a (generalized) closure relation $\vdash$ can conveniently be thought of as an element - an ordered pair $\langle X, y\rangle$ or $\langle X, Y\rangle$ as the case may be-of the set of pairs $\vdash$.) In considering also generalized rule-like conditions, we are envisaging something at the level of sequent-to-sequent rules analogous to the shift from consequence to generalized consequence relations at the level of the premisses and conclusions of such rules. In both cases, the generalizing move consists in dropping a requirement that a condition be expressed by a universal strict Horn formula, as is done at the 'macro' level (of rules) by allowing $n$ to be arbitrary in (12), and at the 'micro' level (of sequents) in defining what it is for a valuation $v$ to be consistent with $\vdash$. (Dropping the 'strict' means allowing $n=0$ as well as $n=1$; this gives precisely the notion of sequent actually employed by Gentzen for the intuitionistic sequent-calculus.)

Digression. Having introduced sequents into the discussion, we can usefully contrast with the Galois connexions between $U$ and $\mathscr{V}$ described in 0.2 another type of Galois connexion which arises naturally in logical studies: namely that with, on the right, $\mathscr{V}$ again (some class of valuations for $U$ ) but this time with the class of all sequents over $U$, on the left. (Galois connexions of the present type are close to explicit in [23], for example.) If we are dealing with sequents of the form $\sigma=\langle X, Y\rangle$, the relation $\varphi$ of this Galois connexion holds between such a $\sigma$ and a valuation $v$ just in case we do not have $v(x)=\mathrm{T}$ for all $x \in X$ while $v(y)=\mathrm{F}$ for all $y \in Y$. (If our sequents are of the (micro-)strict Horn form, apply this definiton for the case of $Y=\{y\}$.) Let us say when this relation obtains between $\sigma$ and $v$ that $\sigma$ holds on $v$. Clearly the valuations consistent with a (generalized) consequence relation $\vdash$ (those making up $\mathscr{V}_{a t}(\vdash)$ ) are precisely those on which each $\sigma \in \vdash$ 
holds, in this sense. We are using the 'consequence' terminology here because we have in mind particularly the case in which $U$ is a language, with $\vdash$ recording the logical relations between its formulas. Continuing in this vein, we might call the unique (generalized) consequence relation $\vdash$ determined by $\mathscr{V}_{0} \subseteq \mathscr{V}: \mathscr{L}_{\log }(\vdash)$. Then the new Galois connexion between, on the left, the class of sequents over $U$ and, on the right, the class $\mathscr{V}$ of all $U$-valuations, is $\left(\mathscr{V}_{a t}, \mathscr{L}_{\log }\right)$. Note that $\mathscr{L}_{\log }\left(\mathscr{V}_{0}\right)$ is always a (generalized) consequence relation, and that $\mathscr{V}_{a t}(\Sigma)$ is defined for any set of sequents $\Sigma$, not just for the case of $\Sigma$ closed under $(\mathbb{R}),(\mathbb{M})$, and $\left(\mathbb{T}^{+}\right)-\mathrm{a}$ (generalized) consequence relation, that is, though the sets on the left which are closed under $\mathscr{L}_{\log } \circ \mathscr{V}_{\text {at }}$ are precisely the (generalized) consequence relations. In speaking of closure under $(\mathbb{R}),(\mathbb{M})$, and $\left(\mathbb{T}^{+}\right)$, we are treating these conditions as rules, as their strict Horn form allows. When generalized rule-like conditions are employed, this possibility is lost. There is, for example, in general no such thing as the least closure relation extending a given closure relation and satisfying (Exchange), because of the disjunctive consequent. This is the 'macro' analogue of the 'micro' point made in 0.1 that there is no notion of the generalized closure of a given subset of $U$. (The 'micro'/'macro' terminology is here being used to mark the same distinction as is marked by the opposition 'horizontal' / vertical' in [15] , following [22].)

The rules $(\mathbb{T})$ and $(\mathbb{M})$ are intimately related to conjunctive and disjunctive combinations on the left, in the above connexions, as we illustrate in the case of non-Horn sequents. If $\sigma_{1}=\langle X \cup\{u\}, Y\rangle$ and $\sigma_{2}=\langle X, Y \cup\{u\}\rangle$, for some $X, Y \subseteq U, u \in U$, then their 'cut-product' $\sigma_{3}=\langle X, Y\rangle$ (which is not uniquely determined by $\sigma_{1}, \sigma_{2}$ ) is a conjunctive combination of $\sigma_{1}$ and $\sigma_{2}$ on the left of the present Galois connexion. Not every pair of sequents have a conjunctive combination, however. Disjunctive combinations always exist, on the other hand, since $\langle X, Y\rangle$ and $\langle W, Z\rangle$ have their 'least common thinning', $\langle X \cup W, Y \cup Z\rangle$, as a disjunctive combination.

Although valuations for $U$ figure on the right of the above Galois connexion ( $\mathrm{s}$, the operations of conjunctive and disjunctive combination for them do not behave as they do in the $(V, T)$ Galois connexions of 0.2 , because the binary relation $\varphi$ of the connexion is now the 'holds on' relation, rather than the 'is true on' relation. For example, conjunctive combinations do not always exist on the right in the present case, even though we are considering all $U$-valuations. By way of proof, take $U$ with at least two distinct elements $a, b$, and consider $v_{1}, v_{2}$ such that $v_{1}(a)=\mathrm{F}=v_{2}(b)$ and $v_{2}(a)=\mathrm{T}=v_{1}(b)$, and sequents $\sigma_{1}=\langle\{a\},\{b\}\rangle$, $\sigma_{2}=\langle\{b\},\{a\}\rangle$. Then $\sigma_{1}$ holds on $v_{1}$ and $\sigma_{2}$ holds on $v_{2}$, but we neither have $\sigma_{2}$ holding on $v_{1}$ nor $\sigma_{1}$ holding on $v_{2}$. Thus the 'Cross-Over Condition' of Prop. 0.3.1 (i) is not satisfied, as we are there told it must be if a Galois connexion has both disjunctive combinations on the left and conjunctive combinations on the right. Since, as we saw in the preceding paragraph, we do in the present case have disjunctive combinations on the left, we conclude that we do not have conjunctive 
combinations on the right. However, as with the $(V, T)$ connexions, when such combinations do exist on the right, they are unique, since if the same sequents hold on $v$ and $v^{\prime}$, we have $v=v^{\prime}$. The reason for this is that for a sequent $\langle\varnothing,\{x\}\rangle$ to hold on $v$ is for the $U$-element $x$ to be true on $v$, and (as before) $U$-valuations verifying precisely the same elements of $U$ are the same. End of Digression.

In fact, the restriction to finite $m$ and $n$ in (12), and the analogous restriction at the 'micro' level are inessential, and we have already been ignoring them in regarding $\left(\mathbb{T}^{+}\right)$and the condition (1) of Example 0.1.1 as rule-like conditions. A more important liberalization we shall make of the above understanding of (generalized) rule-like conditions is to allow ourselves in the addition to $\vdash$ -statements themselves in the $\mathscr{A}_{2}, \mathscr{B}_{3}$ in (12), statements formed with the abbreviative notations ' $\vdash^{\exists}$ ' and ' $\vdash^{c c}$, , which will allow us to 'bury' certain quantifiers from explicit view. They are explained in 0.5. There is yet another dimension along which to consider questions of liberality: namely, the extent to which set-theoretic vocabulary (such as $\cap$ ) enters into the formulation of a condition. Brief mention of the role of such ancillary set-theoretic apparatus will be made in 3.2 .

\subsection{Two Auxiliary Notations}

Given a closure relation $\vdash$ on $U$ we define $X \vdash^{\exists} Y$ to hold (for $X, Y \subseteq U$ ) just in case there exists $y \in Y$ such that $X \vdash y$. Note that $X \vdash^{\exists} Y$ never holds when $Y=\varnothing$. The second piece of auxiliary notation has a more complicated definition, whose rationale will become clear presently. Given a closure relation $\vdash$ as before, we define $X \vdash^{\text {cc }} Y$ to hold just in case: for all $X^{\prime} \supseteq X$, for all $z$, if $X^{\prime}, y \vdash z$ for each $y \in Y$, then $X^{\prime} \vdash z$. (The 'cc' is for 'common consequences', since, thinking of the case in which our closure relation is a consequence relation, the elements $z$ are consequences of each of the $y \in Y$, when taken, one by one, in conjunction with an arbitrary superset $X^{\prime}$ of $X$.)

Although we regard the notations ' $\vdash^{\exists}$ ' and ' $\vdash^{\mathrm{cc}}$ ' simply as abbreviatory aids for the formulation of statements about closure relations, it is not hard to see that the definitions given make $\vdash^{\exists}$ and, under certain circumstances $\vdash^{\text {cc }}$, too, generalized closure relations in their own right, and this is what is done in [23], where it they go under the names $\vdash_{\text {min }}$ and $\vdash_{\text {max }}$, respectively. (See also [7], p.8 for exposition, and p.28 for an interesting application.) As Scott remarks, these are respectively the least and the greatest (by the ordering $\subseteq$ ) generalized closure relations which agree with the given closure relation $\vdash$, where a generalized closure relation $\vdash^{\prime}$ is said to agree with the closure relation $\vdash$ just in case $X \vdash^{\prime} u$ iff $X \vdash u$, for all $X, u$. This remark à propos of $\vdash_{\max }$ requires however a special assumption - which is why we said 'under certain circumstances', above - namely that $\vdash$ is finitary (which is satisfied in Scott's discussion since he actually 
stipulates that $\vdash$ is a relation between finite sets and individual elements). The problem in the general case is that $\vdash^{\text {cc }}$ is not guaranteed to satisfy $\left(\mathbb{T}^{+}\right)$, though it is guaranteed to satisfy $(\mathbb{T})$. (See $[26]$, p.75.) We record some obvious consequences of the definitons of $\vdash^{\exists}, \vdash^{\text {cc }}$ :

Proposition 0.5.1. Given to any closure relation $\vdash$ with underlying set $U$, for all $X, Y \subseteq U$, where $\bar{X}$ is $\{u \in U \mid u \notin X\}$ :

(i) $X$ is $\vdash$-closed iff $X \nvdash^{\exists} \bar{X}$.

(ii) $X$ is a maximal avoider (relative to $\vdash$ ) iff $X \nvdash^{c c} \bar{X}$.

(iii) $X \vdash^{\exists} Y$ iff every $\vdash$-closed superset of $X$ contains some element of $Y$.

(iv) $X \vdash{ }^{\text {cc }} Y$ iff every maximal avoider $\supseteq X$ contains some element of $Y$.

(v) $X \vdash^{\exists} Y$ iff for all $v \in \mathscr{V}_{a l}(\vdash)$ with $v(x)=\mathrm{T}$ for each $x \in X$, we have $v(y)=\mathrm{T}$ for some $y \in Y$.

(vi) For the case in which $\vdash$ is a Lindenbaum closure relation: $X \vdash^{\mathrm{cc}} Y$ iff there exists some $\mathscr{V}$ determining $\vdash$ such that for all $v \in \mathscr{V}$ with $v(x)=\mathrm{T}$ for each $x \in X$, we have $v(y)=\mathrm{T}$ for some $y \in Y$.

We will appeal to parts (i) and (ii) of 0.5.1 on many occasions in what follows, and to parts (ii), (iv), (vi) somewhat less often; part (v) is just (iii) reformulated for the sake of an instructive contrast with (vi). The contrast between $\vdash^{\exists}$ and $\vdash^{c c}$ will loom large in $\S \S 1,2$ as we compare generalized rule-like conditons on closure relations whose classes $\mathscr{V}_{a}(\vdash)$ of consistent valuations are closed under Galois-duals of boolean connectives with conditions requiring only that some class of valuations determining $\vdash$ is thus closed; the latter conditions themselves frequently involve, as we shall also see, an intriguing blend of $\vdash^{\exists}$ and $\vdash^{\text {cc }}$.

\subsection{Tight Sets and the Exchange Property}

In 0.4 we mentioned the traditional association between the Exchange Property and irredundant bases of closed sets. Here we are concerned instead with the irredundancy of a basis in so far as single elements of the $\vdash$-closure of that basis are concerned. With respect to a closure relation $\vdash$ on some set $U$, the elements of $X \subseteq U$ have been called generalized equivalent when for each $x \in X, X \backslash\{x\} \vdash x$. (For applications and further references, see [19] .) What we need for certain purposes below is a refinement of this notion. Given a closure relation or generalized closure relation, let us write $X \vdash^{\text {irr }} y$ (' $X$ irredundantly yields $y^{\prime}$ ) to mean that $X \vdash y$ and for all $x \in X, X \backslash\{x\} \nvdash y$. (Notice the claim that $X \vdash^{\text {irr }} y$ implies but is not implied by conjunction of the claims that $X$ is irredundant relative to $\vdash$ and that $X \vdash y$.) We will call $X \subseteq U$ a tight set relative to a closure or generalized closure relation $\vdash$, with $U$ as underlying set, when for each $x \in X: X \backslash\{x\} \vdash{ }^{\text {irr }} x$. (Except for one occasion in 1.2 , we will be mainly interested in tightness relative to a closure relation.) 
Proposition 0.6.1. For $\vdash$ any a closure relation on $U$, satisfying (Exchange), for any $X \subseteq U, y \in U$, if $X \vdash{ }^{\text {irr }} y$ then $X \cup\{y\}$ is a tight set.

Proof. Under the conditions of 0.6.1, we need to verify that for all $z \in X \cup\{y\},(X \cup\{y\}) \backslash\{z\} \vdash^{\text {irr }} z$. The assumption that $X \vdash^{\text {irr }} y$ gives this conclusion immediately for the case of $z=y$, so suppose that $z \in X$, and let $X_{0}=X \backslash\{z\}$. We are given (1) $X_{0}, z \vdash^{\text {irr }} y$, and are trying to show that $X_{0}, y \vdash{ }^{\text {irr }} z$. Since $X_{0}, z \vdash y$, the condition (Exchange) implies that either (2) $X_{0}, y \vdash z$ or else (3) $X_{0} \vdash y$. Since (3) contradicts (1), it is case (2) which holds, so it remains only to justify the superscripting of 'irr' in (2). The element $y$ is not redundant in yielding $z$ from $X_{0} \cup\{y\}$ since if $X_{0} \vdash z$, then as $X_{0}, z \vdash y$ (the ' $\vdash$ ' part of (1)), we would have, by $(\mathbb{T}), X_{0} \vdash y$ (contradicting the 'irr' part of (1)). So it remains to check that none of the elements of $X_{0}$ are redundant in (2). For a contradiction, then, suppose that we have $x \in X_{0}$ with

$$
X_{0} \backslash\{x\}, y \vdash z .
$$

By the Exchange Property again, (4) would imply that either (5) or (6) holds:

$$
\begin{gathered}
X_{0} \backslash\{x\}, z \vdash y \\
X_{0} \backslash\{x\} \vdash z .
\end{gathered}
$$

We have already seen that $y$ is not redundant in (2), which (6) would imply, and (5) also contradicts (1).

If $\vdash$ is a closure relation which satisfies (Exchange) and has the following Irredundancy Property:

For all $X \subseteq U, y \in U$ : if $X \vdash y$ then for some $X_{0} \subseteq X, X_{0} \vdash{ }^{\text {irr }} y$

then a specification of the tight sets (relative to $\vdash$ ) completely fixes $\vdash$. We can recover $\vdash$ from the collection of tight sets by means of Proposition 0.6.2, whose straightforward proof is omitted. In $\S 3$ we will make use of this fact for the case of finitary closure relations satisfying (Exchange). Finitary closure relations all have the Irredundancy Property, since we can eliminate redundant elements 'one by one' until they are all gone (and so pass from $X$ to $X_{0}$, for any given $y$; see [29] for further discussion). The author does not know whether the broader class (cf. Example 0.1.1) of Lindenbaum closure relations likewise all possess the Irredundancy Property.

Proposition 0.6.2. If $\vdash$ is a closure relation with the Irredundancy Property and the Exchange Property, and $\mathscr{T}$ is the collection of all tight sets relative to $\vdash$, then $X \vdash y$ iff for some $X_{0} \subseteq X, X_{0} \cup\{y\} \in \mathscr{T}$. 
Our next observation on (Exchange) does not impose any further conditions on $\vdash$ :

Proposition 0.6.3. If $\vdash$ is a closure relation satisfying (Exchange), then any maximal avoider relative to $\vdash$ is an absolute maximal avoider.

Proof. Suppose $W$ is a maximal avoider relative to $\vdash$, a closure relation satisfying (Exchange), with underlying set $U$; for example, suppose that $W$ maximally avoids $x \in U$. Let $y$ be any element of $U$ which does not belong to $W$. To show that $W$ is an absolute maximal avoider, it will suffice to show that $W, x \vdash y$, since this implies $W, z \vdash y$ for all $z \notin W$ (by $(\mathbb{T}$ ) and the fact that $W, z \vdash x)$. As $y \notin W$ and $W$ maximally avoids $x$, we have $W, y \vdash x$. So by (Exchange), either $W, x \vdash y$ or else $W \vdash x$; but the latter disjunct contradicts the fact that $W$ maximally avoids $x$.

One moral of Prop. 0.6.3 is that not any stipulation that such-and-such sets are to be amongst the tight sets relative to a finitary closure relation with the Exchange Property will be consistent. While in general the tight sets will form a rather complicated interlocking network, certain patterns of overlap are prohibited:

Example 0.6.4. Suppose that $U=\{a, b, c, d\}$ and we stipulate that $\{a, b, c\}$ and $\{a, b, d\}$ are to be tight, relative to some closure relation (of necessity finitary) we are describing by means of this stipulation. This has the consequences that $\{c\}$ maximally avoids $d$, but does not maximally avoid $a$, since $\{c, d\}$ still 'avoids' $a$ (and $b$ ). Thus while $\{c, d\}$ is an absolute maximal avoider, $\{c\}$ is a non-absolute maximal avoider, and by Prop. 0.6.3, the closure relation described does not satisfy (Exchange).

In the case just described, the stipulation about what were to be amongst the tight sets was not consistent with the closure relation concerned having the Exchange Property, though that stipulation was perfectly consistent in itself (and so does describe a unique $\vdash$ ). Contrast here the stipulation that amongst the tight sets were to be, say, $\{a, b\}$ and also $\{a, b, c\}$ : here there is an internal inconsistency, since the notion of tightness rules out the possibility that one tight set should be a proper subset of another.

Incidentally, the reason we formulate the above descriptions of closure relations by means of stipulations to the effect that such and such sets are to be counted amongst the tight sets is that the least closure relation relative to which certain given sets are tight will in general have other sets tight as a consequence. For example, where $U=\{a, b, c, d, e, f\}$ and $\{a, b, c\}$ as well as $\{c, d, e, f\}$ are tight, we must also have $\{a, b, d, e, f\}$ tight. 
(An analogous 'cut-like' phenomenon is observed for arbitary sets of generalized equivalent elements in [19], p.287.)

\section{$\S 1$. Supervenience and Matching}

\subsection{Two Problems Suggested by the Idea of Supervenience}

Many issues in recent philosophy have turned on some version of the notion of supervenience ( $[16],[17], \mathrm{pp} .14-17)$, which for present purposes is well enough explained thus: a property $y$ is supervenient on a class $X$ of properties if any two individuals agreeing on all the properties in $X$, in the sense that for each such property either both have the property or both lack the property, must also agree on $y$. It does not matter what precisely is to be understood by the term 'property' here, as long as for an arbitrary individual, that individual either has or lacks the property. Thus, think of expressions like 'is ten metres tall' as picking out properties, rather than expressions like 'height' (or 'height-in-metres'), which specifies, rather, a class of properties (including that just mentioned). It will do to think of properties as (not necessarily arbitrary) classes of individuals, and of having (lacking) the property being a matter of belonging to (not belonging to) the class. Where $I$ is our set of individuals, and $U$ our set of properties of individuals, then the claim that $y \in U$ is supervenient on $X$ is the claim that for all $i, j \in I$, if $v_{\imath}(x)=v_{j}(x)$ for each $x \in X$, then $v_{\imath}(y)=v_{j}(y), v_{\imath}$ being the characteristic function of the class of properties possessed by the individual $i$ (and similarly for $v_{j}$ ). Clearly the relation defined thus is a closure relation, and the question arises as to what special features it must have (regardless of $I$ and $U$ ) over and above those features common to all closure relations. To pursue this question we can leave out the reference to $I$ and consider a class $\mathscr{V}$ of valuations, defining a closure relation $\vdash$ on $U$ to be supervenience-determined by $\mathscr{V}$ when for all $v, v^{\prime} \in$ $\mathscr{V}$, all $X \subseteq U, y \in U$ :

$$
X \vdash y \text { if and only if: } v(x)=v^{\prime}(x) \text { for all } x \in X \text {, implies } v(y)=v^{\prime}(y)
$$

Using the binary matching operation $\sim$ from 0.2 (last paragraph) on valuations, we can see that $\vdash$ 's being supervenience-determined by $\mathscr{V}$ amounts to $\vdash$ 's being determined (tout court) by $\mathscr{V} \sim \mathscr{V}=\left\{v \sim v^{\prime} \mid v, v^{\prime} \in \mathscr{V}\right\}$, so the question raised becomes the first of these two problems:

Problem 1.1.1. When is a closure relation $\vdash$ determined by some class of valuations of the form $\mathscr{V} \sim \mathscr{V}$ ?

Problem 1.1.2. When is a closure relation $\vdash$ determined by some class of valuations closed under the operation $\sim$ ? 
Problem 1.1.2 is a special case of 1.1.1, in the sense that if $\mathscr{V}$ is closed under $\sim$ and $\mathscr{V}$ determines $\vdash$, then $\mathscr{V}$ is itself either empty (in which case $X \vdash y$ for all $X, y)$ or else - as will be explained presently -is equal to $\mathscr{V} \sim \mathscr{V}$, and so counts as one kind of case in which $\vdash$ is determined by a class $\mathscr{V}^{\prime}$ of valuations of the form $\mathscr{V} \sim \mathscr{V}$. Of course this is only the special case in which $\mathscr{V}^{\prime}=\mathscr{V}$, whereas Problem 1.1.1 asks after the general case in which $\mathscr{V}^{\prime}$ is $\mathscr{V} \sim \mathscr{V}$ : for some $\mathscr{V}$ or other-no t necessarily for $\mathscr{V}$ as $\mathscr{V}^{\prime}$ itself (though, by an argument we do not give here, $\mathscr{V}$ can always be chosen so that $\mathscr{V} \subseteq \mathscr{V}^{\prime}$ ).

We turn to an explanation of the above claim that unless $\mathscr{V}$ is empty, if $\mathscr{V}$ is closed under $\sim$, then $\mathscr{V}=\mathscr{V} \sim \mathscr{V}$. The hypothesis that $\mathscr{V}$ is closed under $\sim$ amounts to one half of the claim that $\mathscr{V}=\mathscr{V} \sim \mathscr{V}$, namely the inclusion $\mathscr{V} \sim \mathscr{V}$ $\subseteq \mathscr{V}$. To obtain the converse inclusion, note that as long as $\mathscr{V} \neq \varnothing$, we may select $v \in \mathscr{V}$ and observe that $v \sim v=v_{T}$, so since $\mathscr{V}$ is closed under $\sim, v_{T} \in \mathscr{V}$. Now, to show $\mathscr{V} \subseteq \mathscr{V} \sim \mathscr{V}$, take any $v^{\prime} \in \mathscr{V}:$ we may represent $v^{\prime}$ as an element of $\mathscr{V} \sim \mathscr{V}$ because $v^{\prime}=v^{\prime} \sim v_{T}$. (Though we make no special play with the fact, a non-empty class of valuations closed under $\sim$ constitutes a 'boolean group' with respect to that operation: a group, that is, in which every element is its own inverse. The identity element is of course $v_{T}$.)

The two problems raised above ask when a closure relation has a certain 'semantically' characterized feature - a feature characterized, that is, in terms of classes of valuations. The intention is to enquire after a 'syntactic' characterization which will apply in precisely the same cases, a characterization, that is, by means of what in 0.4 we called generalized rule-like conditions, or more generally, collections of such conditions. It is Problem 1.1.2 in which we shall take a special interest in what follows, obtaining a partial solution - restricted in scope to Lindenbaum closure relations - in $\S 3$ (Thms. 3.1.2, 3.2.2). But we offer a few comments here on the harder, more general, Problem 1.1.1.

One generalized rule-like condition satisfied by any superveniencedetermined closure relation is a special case of the Exchange Property

$$
x \vdash y \text { implies either } y \vdash x \text { or } \vdash y \text { (i.e., } \varnothing \vdash y \text { ) }
$$

This is the special case of (Exchange), repeated here for convenience, in which $X=\varnothing$ :

$$
\text { (Exchange) } \quad X, x \vdash y \text { implies } X, y \vdash x \text { or } X \vdash y \text {. }
$$

The general case, including non-empty $X$, is not a condition which is satisfied by all supervenience-determined closure relations. A second condition, not following from (13), which is likewise satisfied by all $\vdash$ determined by some $\mathscr{V}^{\prime}$ of the form $\mathscr{V} \sim \mathscr{V}$ is $(14):$

$$
x, u \vdash y \text { and } x, y \vdash u \text { imply either } u, y \vdash x \text { or } u \vdash y
$$


Justification of the claims that (13) and (14) are satisfied by all superveniencedetermined closure relations may be found in [15], Propositions 3.6, 3.8. Problem 1.1.1 asks for some conditions which are not only, as (13) and (14) are, necessary for $\vdash$ to be supervenience-determined, but also sufficient for this. We have not been able to solve this problem, and conclude these remarks with the observation that both (13) and (14) are special cases of the more general condition

$$
X, x \vdash y \text { and } x, y \vdash u \text { for each } u \in X \text { imply either } X, y \vdash x \text { or } X \vdash y
$$

(13) arises by taking $X=\varnothing$ and (14) by taking $X=\{u\}$; as already remarked, the author does not know whether (15) singles out precisely the superveniencedetermined closure relations.

Digression. Above we suggested that it was things like being ten metres tall that were to count as properties, rather than things like height (-in-metres). Using the more general term 'attribute' from the literature on relational database theory (see the references cited in [15]), we consider properties to be attributes taking, for a given individual, the values $\mathrm{T}$ (rue) and $\mathrm{F}$ (alse), rather than values from whatever range (e.g. particular heights) is appropriate for the attribute in question. Re-interpreting the ' $x$ ', ' $y$ ' in the above definition of supervenience-determination:

$$
X \vdash y \text { if and only if: } v(x)=v^{\prime}(x) \text { for all } x \in X \text {, implies } v(y)=v^{\prime}(y)
$$

to range over arbitrary attributes rather than just bivalent attributes, we get a more general version of the concept, one in terms of which it can be shown ([15], Thm. 4.2) that every closure relation is supervenience-determined by some class of 'valuations'. (For this more general notion, [15] says 'fd-determined', 'fd' abbreviating 'functional dependency'; Problem 1.1.1 was also raised in [15].) End of Digression.

Returning to Problem 1.1.2, we shall consider also, in 1.2, the analogous question for generalized closure relations. (Of course Problem 1.1.1 also lends itself to such a variation.) This turns out to admit of a much simpler solution than the question for closure relations themselves. Since for generalized closure relations $\vdash$ the only class of valuations determining $\vdash$ is $\mathscr{V}_{a t}(\vdash)$, we will also investigate, even when discussing a closure relation $\vdash$, the question of when $\vdash$ is such that $\mathscr{V a t}(\vdash)$ is closed under the matching operation. Our discussion of these questions occupies the remainder of this section and also $\S 3$, with most of $\S 2$ devoted to the corresponding questions for the other operations listed in $\S 0$ as Galois-dual to the more familiar boolean connectives. 


\section{2. (Flip-around) and some Related Conditions}

The solution for Problem 1.1.2 with 'closure' replaced by 'generalized closure' is straightforward. The condition on generalized closure relations we need is:

(Flip-around) $X_{0}, X_{1} \vdash Y_{0}, Y_{1}$ implies $X_{0}, Y_{0} \vdash X_{1}, Y_{1}$ or $X_{0}, Y_{1} \vdash X_{1}, Y_{0}$

(for all $X_{0}, X_{1}, Y_{0}, Y_{1} \subseteq U$ )

Henceforward, such universal quantifications over subsets (and, when lower case letters are used, elements) of $U$ as appears here parenthetically will usually be left implicit in the formulation of similar conditions on (generalized) closure relations. In the following proof, and generally from now on, we use notations such as ' $v(X)=\mathrm{T}$ ' to mean that $v(x)=\mathrm{T}$ for all $x \in X$. (We now have three succinct ways of saying the same thing: $v(X)=\mathrm{T}, X \subseteq T(v)$, and $v \in V(X)$. Concerning the new notation, note that ' $v(X)=\mathrm{T}$ ' and ' $v(X)=\mathrm{F}$ ' are not in general mutually exclusive, since we may have $|X|=0$, or jointly exhaustive, since we may have $|X| \geq 2$.)

Theorem 1.2.1. A generalized closure relation $\vdash$ satisfies (Flip-around) iff Vat $(\vdash)$ is closed under $\sim$.

Proof. 'If'. Suppose $\mathscr{V}_{a}(\vdash)$ is closed under $\sim$ and the consequent of (Flip-around) fails for some $X_{0}, X_{1}, Y_{0}, Y_{1}$. We have to show that this implies that the antecedent of the condition also fails.

Since $X_{0}, Y_{0} \nvdash X_{1}, Y_{1}$ and $X_{0}, Y_{1} \not \forall X_{1}, Y_{0}$, there exist $v, v^{\prime} \in \mathscr{V}_{a t}(\vdash)$ with $v\left(X_{0} \cup Y_{0}\right)=\mathrm{T}, v\left(X_{1} \cup Y_{1}\right)=\mathrm{F}$ and $v^{\prime}\left(X_{0} \cup Y_{1}\right)=\mathrm{T}, v^{\prime}\left(X_{1} \cup Y_{0}\right)=\mathrm{F}$. Now the assumption of closure under $\sim$ implies that $v \sim v^{\prime} \in \mathscr{V}_{a t}(\vdash)$. But $v \sim$ $v^{\prime}\left(X_{0} \cup X_{1}\right)=\mathrm{T}$, since $v\left(X_{0}\right)=v^{\prime}\left(X_{0}\right)=\mathrm{T}$ and $v\left(X_{1}\right)=v^{\prime}\left(X_{1}\right)=\mathrm{F}$; similarly, as $v\left(Y_{0}\right)=\mathrm{T} \neq \mathrm{F}=v^{\prime}\left(Y_{0}\right)$ and $v\left(Y_{1}\right)=\mathrm{F} \neq \mathrm{T}=v^{\prime}\left(Y_{1}\right)$, we have $v \sim v^{\prime}\left(Y_{0} \cup Y_{1}\right)=\mathrm{F}$. These facts imply that $X_{0}, X_{1} \nvdash Y_{0}, Y_{1}$, as desired.

'Only if'. Suppose that $\vdash$ satisfies (Flip-around) and that $v, v^{\prime} \in \mathscr{V}_{a t}(\vdash)$. To show that $\mathscr{V}_{a t}(\vdash)$ is closed under $\sim$, we derive a contradiction from supposing further that $v \sim v^{\prime} \notin \mathscr{V}_{a}(\vdash)$. The latter supposition means that we have $W, Z$ with $W \vdash Z$ but $v \sim v^{\prime}(W)=\mathrm{T}$ while $v \sim v^{\prime}(Z)=\mathrm{F}$. Put $W=X_{0} \cup X_{1}, Z=Y_{0} \cup Y_{1}$, where $v\left(X_{0}\right)=v^{\prime}\left(X_{0}\right)=\mathrm{T}, v\left(X_{1}\right)=v^{\prime}\left(X_{1}\right)=\mathrm{F}, v\left(Y_{0}\right)=\mathrm{T} \neq \mathrm{F}=v^{\prime}\left(Y_{0}\right)$, and $v\left(Y_{1}\right)=\mathrm{F} \neq \mathrm{T}=v^{\prime}\left(Y_{1}\right)$.

Since we have $X_{0}, X_{1} \vdash Y_{0}, Y_{1}$ (alias $W \vdash Z$ ), we can invoke the Flip-around condition and infer that either $X_{0}, Y_{0} \vdash X_{1}, Y_{1}$ or else $X_{0}, Y_{1} \vdash X_{1}, Y_{0}$. But the above assignments of truth-values show that the first of these two possibilities contradicts the assumption that $v \in \mathscr{V}_{\text {at }}(\vdash)$, while the second contradicts the assumption that $v^{\prime} \in \mathscr{V}_{a l}(\vdash)$. 
Note that, in view of the discussion following Thm. 0.2 .1 , we could equally well have replaced ' $\mathscr{V}_{a}(\vdash)$ ' by 'some class of valuations determining $\vdash$ ' or 'every class of valuations determining $\mathscr{V}^{\prime}$; of course such replacements are no longer equivalent in the case in which $\vdash$ is a closure relation.

For the sake of a comparison, taken up in 3.2, with closure relations, we give here a consequence of (Flip-around) pertaining to tight sets. We recall that the latter notion, from 0.6, makes sense for generalized closure relations and that for such a relation $\vdash$, exactly as for a closure relation, a set $Z$ is tight when for all $x \in Z, Z \backslash\{x\} \vdash x$ while for all proper subsets $Y$ of $Z \backslash\{x\}, Y \nvdash x$.

Lemma 1.2.2. For any generalized closure relation on $U$, with respect to which $X \cup Y \subseteq U$ is tight, where $X \cap Y=\varnothing$, if $|Y|=2$, then $X \forall \forall Y$.

Proof. Let $Y$ consist of the two elements $a, b$. Assume $X \cup Y$ tight, and that $X \vdash a, b$. Since $X, a \vdash b$, (T) gives the conclusion that $X \vdash b$, contradicting the tightness of $X \cup Y$.

Theorem 1.2.3. For any generalized closure relation on $U$, which satisfies (Flip-around) and with respect to which $X \cup Y \subseteq U$ is tight, where $X \cap Y=\varnothing$, if $|Y|$ is odd, then $X \vdash Y$.

Proof. Since the hypothesis that $|Y|$ is odd implies that $Y$ is finite, we can proceed by induction on $|Y|$. The basis case is that of $|Y|=1$, which is secured by the fact that $X \cup Y$ is tight. For the inductive step, suppose the result holds for odd $|Y|=n$, and we have $\left|Y^{\prime}\right|=n+2$. Write $Y^{\prime}$ as $Y \cup\{a, b\}$, where $a \neq b$ and $a, b \notin Y$. We must show that $X \vdash Y^{\prime}$, i.e., that $X \vdash Y, a, b$, on the assumption that $X \cup Y^{\prime}$ is tight. By this assumtion and the inductive hypothesis, we have $X, a, b \vdash Y$; so by (Flip-around) either $X, Y \vdash a, b$ or else $X \vdash Y, a, b$. Lemma 1.2.2 rules out the first possibility, so we have the desired conclusion.

We now turn our attention to closure relations, beginning by adorning the condition (Flip-around) with superscripted ' $\exists$ 's:

(Flip-around ${ }^{\exists} \exists$ ) $X_{0}, X_{1} \vdash^{\exists} Y_{0}, Y_{1}$ implies $X_{0}, Y_{0} \vdash^{\exists} X_{1}, Y_{1}$ or $X_{0}, Y_{1} \vdash^{\exists} X_{1}, Y_{0}$

It is then possible to show, in the way that Thm. 1.2.1 does for (Flip-around) itself and generalized closure relations, that a closure relation $\vdash$ satisfies (Fliparound ${ }^{\exists} \exists$ ) iff $\mathscr{V}_{a}(\vdash)$ is closed under $\sim$; the argument is similar to that given for a simpler condition for which we state this result as Thm. 1.2.4. The simplicity consists in reducing the number of superscripted ' $\exists$ 's:

(Flip-around ${ }^{\exists}$ ) $X_{0}, X_{1} \vdash y$ implies $X_{0}, y \vdash^{\exists} x_{1}$ or $X_{0} \vdash y$

It is sometimes convenient to work with a formulation which is intermediate in 
simplicity between (Flip-around ${ }^{\exists}{ }^{\exists}$ ) and (Flip-around ${ }^{\exists}$ ), namely:

(Flip-around ${ }^{\exists}$ ) $X_{0}, X_{1} \vdash y$ implies $X_{0}, y \vdash^{\exists} X_{1}$ or $X_{0} \vdash^{\exists} X_{1}, y$

Obviously the satisfaction (by a given $\vdash$ ) of this condition follows from the satisfaction of (Flip-around ${ }^{\exists}$ ), since if $X_{0} \vdash y$ then there is some element of $X_{1} \cup\{y\}$, namely $y$ (at least) which is in the $\vdash$-closure of $X_{0}$. And conversely, if $\vdash$ satisfies (Flip-around ${ }^{\exists}$ ), $\vdash$ must satisfy (Flip-around ${ }^{\exists}$ ); for suppose that (Flip-around ${ }^{\exists}$ ) is satisfied but we have $X_{1}, x, y$ with $X_{0}, X_{1} \vdash y$ yet neither $X_{0}, y \vdash^{\exists} X_{1}$ nor $X_{0} \vdash y$. Then, appealing to (Flip-around ${ }^{\exists}$ ), since $X_{0}, X_{1} \vdash y$ and $X_{0} \not \nvdash y$, we have $X \vdash^{\exists} U, y$. But again since $X_{0} \not \nvdash y$, any element $u \in X_{1} \cup\{y\}$ for which $X_{0} \vdash u$ must belong to $X_{1}$; and as $X_{0} \vdash{ }^{\exists} X_{1}, y$, there must be some such element. So $X_{0} \vdash^{\exists} X_{1}$, and hence (by (M)) $X_{0}, y \vdash^{\exists} X_{1}$, which was supposed not to be the case. This contradiction shows that (Flip-around ${ }^{\exists}$ ) follows from the more complicated condition (Flip-around ${ }^{\exists}$ ) ; thus the two conditions are equivalent.

In dealing with (Flip-around ${ }^{\exists}$ ) itself, we shall need to employ the concept of the (general) conjunctive combination of a class of valuations (from 0.2).

Theorem 1.2.4. A closure relation $\vdash$ satisfies (Flip-around ${ }^{\Xi}$ ) iff $\mathscr{V}_{a t}(\vdash)$ is closed under $\sim$.

Proof. 'If': Suppose that $\mathscr{V}_{a}(\vdash)$ is closed under $\sim$, but that $\vdash$ does not satisfy (Flip-around ${ }^{\exists}$ ) ; thus, exploiting the equivalence of this condition with (Flip-around ${ }^{\exists}$ ), there are $X, U, y$ with
(1) $X_{0}, X_{1} \vdash y$
(2) $X_{0}, y \nvdash^{\exists} X_{1}$
(3) $X_{0} \not \nvdash^{\exists} X_{1}, y$

By (2), there exists for each $u \in X_{1}$ some $v \in \mathscr{V}_{a l}(\vdash)$, such that $v\left(X_{0}\right)=u(y)=\mathrm{T}$, and $v(u)=\mathrm{F}$. Let $\mathscr{V}_{1}$ be the set of such $v$. Note that $\Delta\left(\mathscr{V}_{1}\right)$ makes the following assignments: $\mathrm{T}$ to all elements of $X_{0} \cup\{y\}, \mathrm{F}$ to all elements of $U$. Similarly, by (3), there exist for each $u^{\prime} \in X_{1} \cup\{y\}$, some $v \in \mathscr{V}_{a t}(\vdash)$ such that $v\left(X_{0}\right)=\mathrm{T}$, while $v\left(u^{\prime}\right)=F$. Collecting these together as $\mathscr{V}_{2}$, we have the following assignments on the part of $\Delta \mathscr{V}_{2}: \mathrm{T}$ to all elements of $X_{0}, \mathrm{~F}$ to all elements of $X_{1} \cup\{y\}$. By Prop. 0.2.3, each of $\Delta \mathscr{V}_{1}, \Delta \mathscr{V}_{2}$ belongs to $\mathscr{T}_{a t}(\vdash)$, so by our assumption of closure under $\sim$ we have $\Delta \mathscr{V}_{1} \sim \Delta \mathscr{V}_{2} \in \mathscr{V}_{\text {at }}(\vdash)$. Since, however, $\Delta \mathscr{V}_{1}$ and $\Delta \mathscr{V}_{2}$ agree on $X_{0}$ (all $\mathrm{T}$ on both valuations) and on $X_{1}$ (all $\mathrm{F}$ on both), they should agree on $y$, in view of (1). But, as we have seen, $\Delta \mathscr{V}_{1}(y)=\mathrm{T}$ while $\Delta \mathscr{V}_{2}(y)=\mathrm{F}$.

'Only if': Suppose that $\mathscr{V}_{a}(\vdash)$ is not closed under $\sim$. Then there exist $v_{1}, v_{2} \in$ $\mathscr{V}_{\text {at }}(\vdash)$ with $v_{1} \sim v_{2} \notin \mathscr{T}_{a d}(-)$. The latter implies that for some $W, y$ such that $W \vdash y$, we have $v_{1} \sim v_{2}(W)=\mathrm{T}, v_{1} \sim v_{2}(y)=\mathrm{F}$. Thus there are $X_{0}, X_{1}$ with $X_{0} \cup X_{1}=W$ such that $v_{1}\left(X_{0}\right)=v_{2}\left(X_{0}\right)=\mathrm{T}, v_{1}\left(X_{1}\right)=v_{2}\left(X_{1}\right)=\mathrm{F}$, while (without loss of generality) $v_{1}(y)=\mathrm{T}$ and $v_{2}(y)=\mathrm{F}$. Since $X_{0}, X_{1} \vdash y$, we can invoke the condition (Flip-around ${ }^{\exists}$ ) to infer that either (1) $X_{0}, y \vdash^{\exists} X_{1}$ or else (2) $X_{0} \vdash y$. 
But (1) contradicts the fact that $v_{1} \in \mathscr{V}_{a l}(\vdash)$, and (2) contradicts the fact that $v_{2} \in \mathscr{V a t}(\vdash)$.

What we would like, for a solution to Problem 1.1.2, is a result like 1.2.4 but making a replacement for (Flip-around ${ }^{\exists}$ ) which is suitable when ' $\mathscr{V}_{a t}(\vdash)$ ' is replaced by 'some class of valuations determining $\vdash$ '. We should first assure ourselves that the reference to (Flip-around ${ }^{\exists}$ ) needs replacing for such a result:

Example 1.2.5. Let $\vdash$ be the consequence relation, on a language containing at least the three distinct atomic formulas ('sentence letters', 'free generators') $a, b$, and $c$, with $\leftrightarrow$ as its sole connective, determined by the class of all $\leftrightarrow$-boolean valuations. (E.g., take the pure equivalential fragment of classical sentential logic.) We know from Prop. 0.3.2 (iii) that this class of valuations is closed under ; but since we have

$$
(a \leftrightarrow b) \leftrightarrow c, a, b \vdash c \text { while }(a \leftrightarrow b) \leftrightarrow c, c \nvdash^{\exists} a, b
$$

and also $(a \leftrightarrow b) \leftrightarrow c \nvdash c$, the closure relation $\vdash$ does not satisfy (Flip-around ${ }^{\exists}$ ). (Take $X_{0}=\{(a \leftrightarrow b) \leftrightarrow c\} X_{1}=\{a, b\}, y=c$.)

Since Example 1.2.5 presents us with a closure relation determined by some $\sim$-closed class of valuations but not satisfying (Flip-around ${ }^{\exists}$ ), we must look elsewhere for a 'syntactic' condition to correspond to determination by some such class. Here we shall consider one particularly promising candidate, similar in form to (Flip-around ${ }^{\exists}$ ), but with different superscripting:

$\left(\right.$ Flip-around $\left.^{\mathrm{cc}}\right) \quad X_{0}, X_{1} \vdash y$ implies $X_{0}, y \vdash^{\mathrm{cc}} X_{1}$ or $X_{0} \vdash{ }^{\mathrm{cc}} X_{1}, y$

A simplification analogous to the passage from (Flip-around ${ }^{\exists}$ ) to (Fliparound $^{\exists}$ ) above, which would reduce the second disjunct of the consequent of (Flip-around $^{\text {cc }}$ ) to ' $X_{0} \vdash y$ ', is not available in the present instance, since it issues in something that does not follow from the condition as formulated here. An early sign of promise on the part of this new condition is recorded in:

Theorem 1.2.6. If a closure relation $\vdash$ is determined by some class of valuations which is closed under $\sim$, then $\vdash$ satisfies (Flip-around ${ }^{\mathrm{cc}}$ ).

Proof. Suppose $\mathscr{V}$, closed under $\sim$, determines $\vdash$, which for some $X, U, y$, we have (1) $X, U \vdash y$ (2) $X, y \nvdash{ }^{c c} U$ (3) $X \nvdash^{c c} U, y$. (2) unpacks into the claim that there is some $X^{\prime} \supseteq X \cup\{y\}$ and there is some $z$, such that $X^{\prime}, u \vdash z$ for each $u \in U$, while $X^{\prime} \not z$. Since $X^{\prime} \not z z$, there is some $v \in \mathscr{V}$ verifying every element of $X^{\prime}$ but not $z$. Thus $v(x)=\mathrm{T}$ for each $x \in X, v(y)=\mathrm{T}$, and $v(z)=\mathrm{F}$. Further, since $v$ verifies every element of $X^{\prime}$, though not $z$, and $X^{\prime}, u \vdash z$ for each $u \in U$, $v(u)=\mathrm{F}$ for each such $u$. A similar unpacking of (3) yields the existence of a 
valuation $v^{\prime} \in \mathscr{V}$ with $v^{\prime}(x)=\mathrm{T}$ for all $x \in \mathscr{V}, v^{\prime}(u)=\mathrm{F}$ for all $u \in U$, and $v^{\prime}(y)=\mathrm{F}$. The valuation $v \sim v^{\prime}$, supposedly also in $\mathscr{V}$, then contradicts (1), since $v$ and $v^{\prime}$ agree on $X$ (all true on both valuations), as well as on $U$ (all false on both), while disagreeing on $y$.

We take up the question of the sufficiency of (Flip-around ${ }^{c c}$ ) for the existence of a determining class of valuations closed under $\sim$ in $\S 3$, here pausing to note the equivalence of this condition to one more similar in form to the condition (Fliparound) on generalized closure relations:

(Flip- around ${ }^{\exists c c}$ ) $X_{0}, X_{1} \vdash^{\exists} Y_{0}, Y_{1}$ implies

$$
X_{0}, Y_{0} \vdash{ }^{\mathrm{cc}} X_{1}, Y_{1} \text { or } X_{0}, Y_{1} \vdash{ }^{\mathrm{cc}} X_{1}, Y_{0}
$$

This new condition just decorates the ' $\vdash$ ' of (Flip-around) with appropriate superscripts, and differs from the condition (Flip-around ${ }^{\exists}{ }^{\exists}$ ) mentioned above $a$ propos of closure relations $\vdash$ for which $\mathscr{V}_{a}(\vdash)$ is closed under $\sim$ in replacing the occurrences of ' $\exists$ ' in the consequent of that condition by 'cc'.

Propositon 1.2.7. A closure relation $\vdash$ satisfies (Flip-around ${ }^{\exists c c}$ ) iff it satisfies $\left(\right.$ Flip-around $\left.^{\mathrm{cc}}\right)$.

Proof. 'If': Suppose $\vdash$ satisfies (Flip-around ${ }^{\text {cc }}$ ) and that $X_{0}, X_{1} \vdash^{\exists} Y_{0}, Y_{1}$ for some $X_{0}, X_{1}, Y_{0}, Y_{1}$. Then for some $y \in Y_{0} \cup Y_{1}$, we have $X_{0}, X_{1} \vdash y$, and so by (Flip-around ${ }^{c c}$ ), either (1) $X_{0}, y \vdash^{c c} X_{1}$ or (2) $X_{0} \vdash^{c c} X_{1}, y$. Suppose $y \in Y_{0}$. Then from (1) by (M) we obtain: $X_{0}, Y_{0} \vdash{ }^{c c} X$; thus by (M) again we have $X_{0}, Y_{0} \vdash{ }^{\mathrm{cc}} X_{1}, Y_{1}$. Likewise, from (2) we obtain $X_{0} \vdash{ }^{c c} X_{1}, Y_{0}$ and hence $X_{0}, Y_{1} \vdash{ }^{c c} X_{0}, Y_{0}$. So either way, on the supposition that $y \in Y_{0}$, we have the desired conclusion: either $X_{0}, Y_{0} \vdash{ }^{\mathrm{cc}} X_{1}, Y_{1}$ or $X_{0}, Y_{1} \vdash{ }^{\mathrm{cc}} X_{1}, Y_{0}$. Suppose, on the other hand, that $y \in Y_{1}$. Then from (1) by successive appeals to $(\mathbb{M})$ we get that $X_{0}, Y_{1} \vdash{ }^{c c} X_{1}$, and that $X_{0}, Y_{1} \vdash{ }^{c c} X_{1}, Y_{0}$, while from (2) we have, similarly, $X_{0} \vdash{ }^{\mathrm{cc}} X_{1}, Y_{1}$, and thus $X_{0}, Y_{0} \vdash{ }^{\mathrm{cc}} X_{1}, Y_{1}$. So for this supposition, too, we obtain the conclusion that $X_{0}, Y_{0} \vdash{ }^{\mathrm{cc}} X_{1}, Y_{1}$ or $X_{0}, Y_{1} \vdash{ }^{\mathrm{cc}} X_{1}, Y_{0}$.

'Only if': Setting $Y_{0}=\{y\}, Y_{1}=\varnothing$, in (Flip-around ${ }^{\exists c c}$ ) gives the condition (Flip-around ${ }^{\mathrm{cc}}$ ).

We will consider in a moment the status of a condition like (Fliparound ${ }^{\exists c c}$ ) but with the initial ${ }^{\prime} \vdash^{\exists}$, replaced by ' $\vdash^{c c}$ '. Before doing so, we should record a fact about $\left(\right.$ Flip-around ${ }^{\mathrm{cc}}$ ) which bears on the status of maximal avoiders relative to a closure relation satisfying that condition (equivalently, satisfying (Flip-around ${ }^{\exists c c}$ ). It is the connexion between maximal avoiders and the relation $\vdash^{\mathrm{cc}}$ given in Prop. 0.5.1 (ii) that makes this observation a timely one to make at this point.

The pertinent observation is that every closure relation satisfying (Flip- 
around $^{\text {cc }}$ ) has the Exchange Property, from 0.4; for convenience we repeat the relevant condition here, with minor re-lettering, as well as (Flip-around ${ }^{\text {cc }}$ ) itself

(Exchange) $\quad X_{0}, u \vdash y$ implies $X_{0}, y \vdash u$ or $X_{0} \vdash y$.

$\left(\right.$ Flip-around $^{\mathrm{cc}}$ ) $X_{0}, X_{1} \vdash y$ implies $X_{0}, y \vdash{ }^{\mathrm{cc}} X_{1}$ or $X_{0} \vdash{ }^{\mathrm{cc}} X_{1}, y$

To obtain (Exchange) from (Flip-around ${ }^{\text {cc }}$ ) put $\{u\}$ for $X_{1}$ and suppose that $X_{0}, u \vdash y$. From the special case of (Flip-around ${ }^{c c}$ ) arising from the substitution just indicated, we infer that either $X_{0}, y \vdash u$ or else $X_{0} \vdash^{c c} u, y$. But given the assumption that $X_{0}, u \vdash y$, this second disjunct implies (by $(\mathbb{T})$ ) that $X_{0} \vdash y$. So if $X_{0}, u \vdash y$ then either $X_{0}, y \vdash u$ or $X_{0} \vdash y$. (Note that only $(\mathbb{T})$, and not the full strength of $\left(\mathbb{T}^{+}\right)$- which, as remarked in 0.5 , may not be available for $\vdash^{\text {cc }}$ - has been invoked here.) Notice incidentally, that a similar argument shows also that (Flip-around ${ }^{\exists}$ ) has (Exchange) as a consequence. However, we record here only what we have found for (Flip-around ${ }^{\mathrm{cc}}$ ):

Proposition 1.2.8. Every closure relation satisfying (Flip-around ${ }^{\mathrm{cc}}$ ) satisfies (Exchange).

The question of whether the converse of this result is also correct will be settled (negatively) in Coro. 3.1.8; for the moment we draw a further conclusion from what we have found:

Corollary 1.2.9. Relative to a closure relation satisfying (Flip-around ${ }^{\mathrm{cc}}$ ), all maximal avoiders are absolute maximal avoiders.

Proof. From Props. 0.6.2, 1.2.8.

Let us now formulate the condition described above and relate its import to what happens wiht maximal avoiders.

(Flip-around ${ }^{\mathrm{cccc}}$ ) $X_{0}, X_{1} \vdash^{\mathrm{cc}} Y_{0}, Y_{1}$ implies $X_{0}, Y_{0} \vdash{ }^{\mathrm{cc}} X_{1}, Y_{1}$ or $X_{0}, Y_{1} \vdash{ }^{\mathrm{cc}} X_{1}, Y_{0}$

For this new principle, we have a result corresponding to the converse of what Thm. 1.2.6 says about (Flip-around ${ }^{\mathrm{cc}}$ ) :

Theorem 1.2.10. If a Lindenbaum closure relation satisfies (Flip-around ${ }^{\mathrm{cccc}}$ ) then it is determined by some class of valuations which is closed under $\sim$.

A proof for this result can be obtained by adapting the argument given in $\S 3$ for Prop. 3.1.4, to show that for each $n \geq 2$ the conditions called in that section 
(Match) $)_{n}$ follow from (Flip-around ${ }^{\text {cccc }}$ ): a difficulty for the inductive part of a similar proof by induction on $n$ for the condition (Flip-around ${ }^{\mathrm{cc}}$ ) itself raised in the discussion leading up to Example 3.2.1 does not arise for the present principle.

(Flip-around $^{\text {cccc }}$ ) does not solve Problem 1.1.2, however, even restricted to the case of Lindenbaum closure relations, because the converse of Thm. 1.2.10 is false. This is most easily shown by means of a small example:

Example 1.2.11. Let $U$ be the five-element set $\{a, b, c, d, e\}$ and $\vdash$ be the closure relation satisfying (Exchange) on $U$ fixed by stipulating that $U$ is the only tight set. By Prop. 0.6.2, this stipulation fixes $\vdash$ uniquely. More explicitly: $\vdash$ is the least closure relation on $U$ satisfying $a, b, c, d \vdash e ; b, c, d, e \vdash a$; etc. Note that for $X \subseteq U, y \in U$, we have: $X \vdash y$ iff $|X|=4$ or $y \in X$.

We will show that the $\vdash$ of Example 1.2.11 is determined by a class of valuations closed under $\sim$ (and therefore, satisfies (Flip-around ${ }^{\mathrm{cc}}$ ), by Thm. 1.2.7) but does not satisfy (Flip-around ${ }^{\text {cccc }}$ ) : therefore the latter condition is too strong to figure in the solution to Problem 1.1.2. The following is easily proved, using the characterization of $\vdash$ at the end of 1.2 .11 for part (i) and an examination of case-types for (ii):

Proposition 1.2.12 (i) The closure relation of Example 1.2.11 is determined by the class of $\mathscr{V}$ of valuations for $U$, where

$$
\mathscr{V}=\{v \mid v(x)=\mathrm{F} \text { for either } 2 \text { or } 4 \text { elements of } U\}
$$

(ii) This class $\mathscr{V}$ is closed under $\sim$.

To bring this description to bear on the question of the excessive strength of (Flip-around ${ }^{c c c c}$ ), we should note that the maximal avoiders for the $\vdash$ of Example 1.2.11 are the three-element subsets of $\{a, b, c, d, e\}$, whose characteristic functions entered the $\mathscr{V}$ of Prop. 1.2.12 as those $v$ with $v(x)=\mathrm{F}$ for 2 elements of $U$. (The other members of $\mathscr{V}$, falsifying instead four elements, are needed because for closure under $\sim$.)

Corollary 1.2.13. The converse of Thm. 1.2 .10 is false.

Proof. We use the closure relation $\vdash$ of Example 1.2.11 to provide a counterexample. By Prop. 1.2.12, we know that $\vdash$ is determined by a class of valuations closed under $\sim$. To show that (Flip-around ${ }^{\text {cccc }}$ ) is not satisfied by $\vdash$, consider the instance of that condition with $X_{0}=X_{1}=\varnothing, Y_{0}=\{a, b\}, Y_{1}=\{c\}$ :

$$
\vdash^{\mathrm{cc}} a, b, c \text { implies } a, b \vdash c \text { or } c \vdash^{\mathrm{cc}} a, b
$$


The antecedent here is true, since every maximal avoider relative to $\vdash$ contains at least one of $a, b, c$ (Prop. 0.5.1 (iv)). But both disjuncts of the consequent are false: the valuation (in $\mathscr{V}$ ) assigning $\mathrm{T}$ to $a, b, d$ and $\mathrm{F}$ to $c, e$, refutes the first; and the fact that $\{c, d, e\}$ is a maximal avoider refutes the second.

Without the superscripted 'cc's, the condition we have just seen not to be satisfied by all closure relations determined by $\sim$-closed classes of valuations is just the condition (Flip-around) on generalized closure relations which Thm. 1.2.1 assures us is satisfied by all (and indeed only) generalized closure relations which are thus determined. So the question arises as to what becomes of the counterexample figuring in the proof of 1.2.13 when the 'cc's are dropped and ' $\vdash$ ' is interpreted as the generalized closure relation (on $\{a, b, c, d, e\}$ determined by the class $\mathscr{V}$ of valuations described in 1.2.12. The answer is that the antecedent of the corresponding appeal to (Flip-around), namely: $\vdash a, b, c$, is no longer true, in view of the $v \in \mathscr{V}$ which assigns $\mathrm{F}$ to each of $a, b, c$ and (say) $d$. This valuation did not spoil the proof of 1.2.12 itself because it is not the characteristic function of a maximal avoider.

The valuation just mentioned, though not the characteristic function of a maximal avoider, is the characteristic function of a $\vdash$-closed set (since it is a valuation consistent with $\vdash$ ). This suggests that we should modify the condition (Flip-around ${ }^{\mathrm{cccc}}$ ) to keep the 'cc's in the consequent, while changing the 'cc' in the antecedent to ' $\exists$ ' (in view of parts (i) and (ii) of Prop. 0.5.1). But of course we have already seen that the result of this modification - called (Flip-around ${ }^{\exists \text { cc }}$ ) above-is equivalent to the originally promising condition (Flip-around ${ }^{\mathrm{cc}}$ ) itself, whose status is on hold until $\S 3$.

We can summarize the morals of Example 1.2.11 in a more general seting, by raising two questions:

(16) If $\vdash$ is a closure relation determined by some $\sim$-closed class of valuations, must the class of characteristic functions of maximal avoiders relative to $\vdash$ be closed under $\sim$ ?

(17) If $\vdash$ is a closure relation determined by some $\sim$-closed class of valuations, must the class of characteristic functions of $\vdash$-closed sets (i.e., the class $\mathscr{V a t}(\vdash))$ be closed under $\sim$ ?

Proposition 1.2.14. The answers to questions (16) and (17) are both negative.

Proof. We obtain a negative answer to both questions by consideration of the closure relation $\vdash$ of Example 1.2.11. The characteristic functions of maximal avoiders $\{a, b, c\}$ and $\{a, d, e\}$ have as their match the characteristic function of 
$\{a\}$ : not a maximal avoider, settling Question (16). This shows that we can have distinct maximal avoiders the match of whose characteristic functions is not the characteristic function of a maximal avoider, which is more than is needed to settle (16). In fact a simpler consideration shows that as long as a closure relation satisfying the antecedent of (16) has some maximal avoider $W$, we have a negative answer, by taking the match of the characteristic function of $W$ with itself, thereby obtaining the characteristic function of $U$ : not a maximal avoider. We now use subscript notation to show which sets a valuation is the characteristic function of. As $\{a, b\}$ and $\{a\}$ are both $\vdash$-closed, we have $v_{a}, v_{a, b} \in \mathscr{V}_{a t}(\vdash)$. But $v_{a} \sim v_{a, b}=v_{a, c, d, e}$, which is not consistent with $\vdash$ as it falsifies $b$ even though $a, c$, $d, e-b$. So the answer to Question (17) is also no.

It would also have been possible to obtain these negative answers, as well as Coro. 1.2.13, from a consideration of a closure relation determined by some class of valuations closed under $\sim$, namely the consequence relation determined by the class of all $\leftrightarrow$-boolean valuations, as presented in Example 1.2.5. We have here a range of consequence relations, in fact, since that Example addressed any language with at least three distinct atomic formulas. Letting $\vdash$ be any one such, and letting $\vdash^{\text {gcr }}$ be the generalized consequence relation on the same language determined by the same class of valuations, it may seem that a difficulty arises for establishing Coro. 1.2.13 on the basis of this example, in view of the equivalence of $X \vdash^{c c} Y$ (defined as usual in terms of the closure relation $\vdash$ ) and $X \vdash^{\text {gcr }} Y$. For, given such an equivalence, the condition (Flip-around ${ }^{\text {cccc }}$ ) for $\vdash$ reduces to (Flip-around) for $\vdash^{\mathrm{gcr}}$, and we know from Thm. 1.2.1 that the generalized closure relations satisfying the latter condition are precisely those determined by some $\sim$-closed class of valuations. Thus we would not expect to find here an example of a (Lindenbaum) closure relation determined by some such class though not satisfying (Fliparound $^{\mathrm{cccc}}$ ).

However, the mooted equivalence between $X \vdash^{c c} Y$ and $X \vdash^{\text {gcr }} Y$ on which these negative remarks are postulated does not quite hold universally, its precise extent being indicated by:

Proposition 1.2.15. With $\vdash^{\mathrm{cc}}$ and $\vdash^{\mathrm{gcr}}$ as above, we have, for all $X, Y$ : $X \vdash^{\mathrm{cc}} Y$ if and (provided $Y \neq \varnothing$ ) only if $X \vdash^{\text {gcr }} Y$.

Proof. 'If': Suppose $X \vdash^{\text {gcr }} Y$ but $X \nvdash^{\text {cc }}$; thus there are $X^{\prime} \supseteq X$ and $z$ with (1) $X^{\prime}, y \vdash z$ for all $y \in Y$ while (2) $X^{\prime} \not \nvdash z$. In view of (2) we have some $\leftrightarrow$ -boolean valuation $v$ with $v\left(X^{\prime}\right)=\mathrm{T}$ and $v(z)=\mathrm{F}$, which in view of (1) means that $v(Y)=\mathrm{F}$. Since $X^{\prime} \supseteq X, v$ provides a counterexample to the original supposition that $X \vdash^{\text {gcr }} Y$.

'Only if': Suppose that $X \vdash^{c c} Y$ for $Y \neq \varnothing$, while $X \nvdash^{\text {gcr }} Y$. Thus there is some $\leftrightarrow$-boolean valuation $v$ with $v(X)=\mathrm{T}, v(Y)=\mathrm{F}$. Since $Y \neq \varnothing$, we may choose 
some element $z \in Y$ and define $X^{\prime}=X \cup\{y \leftrightarrow z \mid y \in Y\}$. We can conclude (1) $X^{\prime}, y \vdash z$ for all $y \in Y$, and (2) $X^{\prime} \not z$. We have (1) because for each $y: y \leftrightarrow z, y \vdash z$. We have (2) because, since $v(y)=v(z)=\mathrm{F}$ for all $y \in Y$ and $v$ is $\leftrightarrow$-boolean, $v(y \leftrightarrow z)=\mathrm{T}$ for each $y \in Y$; therefore, since we already knew $v(X)=\mathrm{T}$, we have $v\left(X^{\prime}\right)=\mathrm{T}$. Since $v(z)=\mathrm{F},(2)$ is established. And (1) and (2) together contradict our supposition that $X \vdash^{\mathrm{cc}} Y$.

The proviso $(Y \neq \varnothing)$ in here is essential not just to the above proof but to the claim being proved. That is, we can have $X \vdash^{\text {cc }} \varnothing$ without $X \vdash^{\text {gcr }} \varnothing$. Unpacking the definition of $\vdash^{\mathrm{cc}}$, we find that $X \vdash^{\mathrm{cc}} \varnothing$ holds just in case $C_{\vdash}(X)=U$. Thus, for example, it holds in the case in which $X=U$, while we do not have in this case $X \vdash{ }^{\text {gcr }} \varnothing$, since $v_{T}$ is $\leftrightarrow$-boolean. (Cf. Thm. 2.3.2.)

We can exploit this lacuna in the would-be equivalence of $\vdash^{\mathrm{cc}}$ with $\vdash^{\text {gcr }}$ to obtain a counterexample to (Flip-around ${ }^{\mathrm{cccc}}$ ) in the present choice (s) of $\vdash$. To do so, first note that where $X$ is the set of all atomic formulas in the language of $\vdash, C_{\vdash}(X)=U$. Let $a$ be one atomic formula and let $X_{0}=X \backslash\{a\}$; thus $X_{0}, a \vdash^{\text {cc }} \varnothing$ (even though $X_{0}, a \nvdash^{\text {gcr }} \varnothing$ ). For the reader's convenience, we repeat the condition

(Flip-around ${ }^{\mathrm{cccc}}$ ) $X_{0}, X_{1} \vdash^{\mathrm{cc}} Y_{0}, Y_{1}$ implies $X_{0}, Y_{0} \vdash^{\mathrm{cc}} X_{1}, Y_{1}$ or $X_{0}, Y_{1} \vdash^{\mathrm{cc}} X_{1}, Y_{0}$ so that it is easy to see that taking $Y_{0}=Y_{1}=\varnothing, X_{1}=\{a\}$, (Flip-around ${ }^{\text {cccc }}$ ) delivers from what we have established $X_{0}, a \vdash^{\text {cc }} \varnothing$, the conclusion $X_{0} \vdash a$. (We have suppressed the 'cc' superscript since only one element appear on the right, and also the redundant disjunction, disjoining this conclusion with itself.) Since for our present choice of $\vdash, X_{0} \forall a$, this shows that the consequence relation (on any language as in 1.2.5) determined by the class of all $\leftrightarrow$-boolean valuations does not satisfy (Flip-around ${ }^{\text {cccc }}$ ), the fact that this class of valuations is closed under notwithstanding.

Returning to Questions (16) and (17) above, we saw (in the proof of Prop. 1.2.14) that a negative answer to (16) was forthcoming for a very wide range of closure relations, which includes the relations $(\mathrm{s}) \vdash$ of our recent discussion, while the negative answer to (17) exploited particular features of the closure relation of Example 1.2.11. We can use the current $\vdash$ to the same effect, however, finding valuations consistent with $\vdash$ whose match is not $\vdash$-consistent:

Example 1.2.16. With $U$ and $\vdash$ as in Example 1.2.5, we have $C_{\vdash}(\{a, b\})$ and $C_{\vdash}(\{c\}) \vdash$-closed while for the match, $v$, of the characteristic functions of these two sets, we have

$$
v(a \leftrightarrow(b \leftrightarrow c))=v(b \leftrightarrow c)=\mathrm{T}
$$

since neither of the formulas exhibited belongs to either of $C_{\vdash}\left(\{a, b\}, C_{\vdash}(\{c\})\right.$. Since $(a \leftrightarrow(b \leftrightarrow c)),(b \leftrightarrow c) \vdash a$, we should have to have $v(a)=\mathrm{T}$ for $v$ to belong 
to $\mathscr{V}_{a t}(\vdash)$. Yet $v(a)=\mathrm{F}$, since $a$ belongs to the first but not the second of our two sets.

We will return to the question of how precisely maximal avoiders and $\vdash$ -closed sets need to be related to answer the question (Problem 1.1.2) of which closure relations are determined by $\sim$-closed classes of valuations in $\S 3$. The analogous question in respect of other operations on valuations which are Galoisdual to boolean sentence connectives in the way that $\sim$ is dual to $\leftrightarrow$ is in many cases considerably easier, and we devote $\S 2$ to pursuing these other cases. The results are interesting in their own right, as well as being (in some cases) suggestive of a strategy which answers the question just raised for $\sim$.

\section{§ 2. Closure under Operations Galois-dual to Various Boolean Connectives}

We will consider, for the reasons just explained, questions of determination (of closure and generalized closure relations) by classes of valuations closed under operations Galois-dual to the boolean sentence connectives other than $\leftrightarrow$ which were listed in 0.2 (as well as some further examples in 2.5), returning to the case of $\sim$ (dual to $\leftrightarrow$ ) in $\S 3$. This is a partial survey, especially in respect of closure relations, rather than a comprehensive theory dealing with the Galois duals of arbitrary truth-functions: it may be thought of as supplying data for such a general theory, however (and some remarks on the general case - for closure relations-appear under 2.5 , and for the easier case of arbitrary generalized closure relations in 2.6).

\subsection{Negative Objects}

Recall from 0.2 that the operation dual to $\neg$ gives for a valuation $v$ the 'complementary' valuation $\bar{v}$ defined by $\bar{v}(u)=\mathrm{T}$ iff $v(u)=\mathrm{F}$ for all $u \in U$. For a generalized closure relation $\vdash$ we have the (rule-like) condition:

(Reverse)

$X \vdash Y$ implies $Y \vdash X$

and the result

Theorem 2.1.1. A generalized closure relation $\vdash$ satisfies (Reverse) iff $\mathscr{V}_{\text {at }}(\vdash)$ is closed under ${ }^{-}$.

Proof. 'If': Suppose that $\mathscr{V}_{a t}(\vdash)$ is closed under ${ }^{-}$and that $Y \nvdash X$, with a view to showing that $X \not \nvdash Y$. Since $Y \not \nvdash X$, we have $v \in \mathscr{V}_{a t}(\vdash)$ with $v(Y)=\mathrm{T}$, $v(X)=\mathrm{F}$. Then $\bar{v}$ belongs to $\mathscr{V}_{a t}(\vdash)$, and since $\bar{v}(X)=\mathrm{T}$ and $\bar{v}(Y)=\mathrm{F}$, this gives the desired conclusion.

'Only if': Suppose that $\vdash$ satisfies (Reverse) but that $\mathscr{T}_{a}(\vdash)$ is not closed under 
-. Thus we have some $v \in \mathscr{V}_{a t}(\vdash)$ but $\bar{v} \notin \mathscr{V}_{a t}(\vdash)$, so that there are $X, Y$ with $X \vdash Y, \bar{v}(X)=\mathrm{T}, \bar{v}(Y)=\mathrm{F}$. Then $v(Y)=\mathrm{T}$ while $v(X)=\mathrm{F}$, contradicting the fact that $v \in \mathscr{V}_{a d}(\vdash)$ and $Y \vdash X$, the latter following from the fact that $X \vdash Y$ by the assumption that satisfies (Reverse).

Now we lock at closure relations $\vdash$ and the condition that $\mathscr{V}_{a t}(\vdash)$ is closed under ${ }^{-}$. The condition on $\vdash$ we need is

(Reverse ${ }^{\exists}$ ) $\quad X \vdash y$ implies $y \vdash^{\exists} X$

An equivalent condition would be the more complicated:

(Reverse ${ }^{\exists}$ ) $X \vdash^{\exists} Y$ implies $Y \vdash^{\exists} X$

which has (Reverse ${ }^{\exists}$ ) as a consequence (taking $Y=\{y\}$ ), and which follows from (Reverse ${ }^{\exists}$ ) since if $X \vdash^{\exists} Y$ then for some $y \in Y$ we have $X \vdash y$, so $y \vdash^{\exists} X$ by (Reverse $^{\exists}$ ), whence $Y \vdash^{\exists} X$ by (M).

Theorem 2.1.2. A closure relation $\vdash$ satisfies $\left(\right.$ Reverse $\left.^{\exists}\right)$ iff $\mathscr{T}_{a l}(\vdash)$ is closed under ${ }^{-}$.

Proof. 'If': Suppose that $\mathscr{V}_{a l}(\vdash)$ is closed under ${ }^{-}$and that $y \not{ }^{\exists} X$, with a view to concluding that $X \not \nvdash y$. Since $y \not{ }^{\exists} X$, there are valuations $v_{x} \in \mathscr{V}_{a l}(\vdash)$ for each $x \in X$, with $v_{x}(y)=\mathrm{T}, v_{x}(x)=\mathrm{F}$. Let $v$ be the conjunctive combination of such $v_{x} ; v \in \mathscr{V}_{a l}(\vdash)$ by Prop. 0.2.3. Since $v(x)=\mathrm{F}$ for each $x \in X$ while $v(y)=$ $\mathrm{T}$, and $\mathscr{V}_{a t}(\vdash)$ is supposed to be closed under ${ }^{-}$, we have $\bar{v} \in \mathscr{V}_{a t}(\vdash)$ with $\bar{v}(X)=\mathrm{T}$ and $\bar{v}(y)=\mathrm{F}$, showing that $X \not \nvdash y$.

'Only if': If $\vdash$ satisfies (Reverse ${ }^{\exists}$ ) but $\mathscr{V}_{a t}(\vdash)$ is not closed under ${ }^{-}$then we have some $v \in \mathscr{V}_{a l}(\vdash)$ with $\bar{v} \notin \mathscr{V}_{a t}(\vdash)$, so that for some $X, y: X \vdash y, \bar{v}(X)=\mathrm{T}$ and $\bar{v}(y)=\mathrm{F}$. Then $v(y)=\mathrm{T}$ while $v(X)=\mathrm{F}$, which contradicts the result of invoking (Reverse ${ }^{\exists}$ ) : $y \vdash^{\exists} X$.

For a result with 'some class of valuations' replacing ' $\mathscr{T}$ a $(\vdash)$ ' here, we make what will come to be something of a standard alteration to (Reverse), analogous to the move from (Flip-around) to (Flip-around ${ }^{\exists c c}$ ): superscript the ' $\vdash$ ' the antecedent with ' $\exists$ ' and those in the consequent with 'cc'. We will refer to this way of obtaining a generalized rule-like condition on closure relations from such a condition on generalized closure relations as the ' $\exists$ cc' strategy; the aim is that the condition obtained should be necessary and sufficient for closure relations or at least Lindenbaum closure relations satisfying it to be determined by a class of valuations closed under whatever operation the original condition guaranteed $\mathscr{V}_{a t}(\vdash)$ to be closed under for precisely such generalized closure relations $\vdash$ as satisfied that original condition. (This raises the question of where we get the condition on generalized closure relations - in the present instance, (Reverse) - to 
begin with. Some remarks on this question will be found in 2.5 below.) Implementing the strategy in the present instance gives us the following condition, weaker than (Reverse ${ }^{\exists}$ ):

(Reverse ${ }^{\exists c c}$ ) $X \vdash^{\exists} Y$ implies $Y \vdash^{c c} X$

Just as (Flip-around ${ }^{\exists c c}$ ) can be simplified to the equivalent (by Prop. 1.2.7) condition (Flip-around ${ }^{\mathrm{cc}}$ ), so the above condition can be simplified to

(Reverse $^{\mathrm{cc}}$ ) $\quad X \vdash y$ implies $y \vdash{ }^{\mathrm{cc}} X$

A straightforward argument along the lines of the proof of 1.2.7 shows $\left(\right.$ Reverse $^{\exists c c}$ ) and $\left(\right.$ Reverse $^{c c}$ ) to be satisfied by precisely the same closure relations. (Yet another equivalent condition would be: $X \vdash u$ and $Y \vdash u$ together imply $Y \vdash{ }^{c c} X$.)

Since a $\vdash^{\text {cc }}$-statement follows from the corresponding $\vdash^{\exists}$-ststatement, it is clear that (Reverse ${ }^{\exists c c}$ ), and hence the equivalent (Reverse ${ }^{c c}$ ), follows from (Reverse ${ }^{\exists}$ ). But the above claim that the former conditions are weaker than (Reverse ${ }^{\exists}$ ) requires us to show that the converse implication fails. We establish this with the aid of an example, as we did for (Flip-around ${ }^{\mathrm{cc}}$ ) and (Flip-around ${ }^{\exists}$ ) in Example 1.2.5. Let $\vdash$ be the consequence relation determined by the class of all $\neg$-boolean valuations on a language (closed under $\neg$ ) containing at least the distinct atomic formulas $a, b$. Then $a, \neg a \vdash b$ but $b \nvdash^{\exists} a, \neg a$. But the cited class of valuations is closed under ${ }^{-}$, by Prop. 0.3.2 (v), so a closure relation can be determined by a class of valuations closed under ${ }^{-}$without satisfying (Reverse ${ }^{\exists}$ ). But as Thm. 2.1.3 will show, there is no such possibility for (Reverse ${ }^{c c}$ ), so $\left(\right.$ Reverse $^{c c}$ ) does not imply (Reverse ${ }^{\exists}$ ). (One can easily check for the above example, for instance, that $b \vdash{ }^{c c} a, \neg a$.)

The role played by maximal avoiders in the following proof forces a restriction in the scope of the envisaged result to Lindenbaum closure relations:

Theorem 2.1.3. A Lindenbaum closure relation $\vdash$ satisfies $\left(\right.$ Reverse $\left.{ }^{\mathrm{cc}}\right)$ iff some class of valuations which determines $\left\llcorner\right.$ is closed under ${ }^{-}$.

Proof. 'If': Suppose $\mathscr{V}$ is closed under ${ }^{-}$and determines $\vdash$, and that $y \not{ }^{c c} X$ with a view to concluding that $X \not \nvdash y$. Since $y \not{ }^{c c} X$, there is some $Y \supseteq\{y\}$ and some $z$ with $Y, x \vdash z$ for each $x \in X$ while $Y \nvdash z$, and therefore some $v \in \mathscr{V}$ with $v(Y)=\mathrm{T} v(X)=\mathrm{F}$. Thus, $\bar{v}(X)=\mathrm{T}$, and (since $y \in Y$ ) $\bar{v}(y)=\mathrm{F}$, showing (since $\mathscr{V}$ is closed under ${ }^{-}$) that $X \not \nvdash y$.

'Only if': We take $\mathscr{V}$ to be the class of characteristic functions of maximal avoiders relative to $\vdash$, together with the characteristic functions of their complements. This guarantees that $\mathscr{V}$ is closed under ${ }^{-}$, but some further work is needed to show that $\mathscr{V}$ determines $\vdash$. If $X \nvdash y$, then, since $\vdash$ is a Lindenbaum closure relation, we have, by Thm. 0.2.2, $v \in \mathscr{V}$ with $v(X)=\mathrm{T}, v(y)=\mathrm{F}$, and similarly, if $X \vdash y$ and 
$v(X)=\mathrm{T}$, we have $v(y)=\mathrm{T}$ for the case in which $v$ is the characteristic function of a maximal avoider. But we must establish this for the new case in which $v$ is the characteristic function of the complement $\bar{Y}(=U \backslash Y)$ of a set $Y$ maximally avoiding (say) $y$. Suppose then, that, for $X$, $y$ such that $X \vdash y$, while $v_{\bar{Y}}(X)=\mathrm{T}$, we have $v_{\bar{Y}}(y)=\mathrm{F}$. Thus $X \subseteq \bar{Y}$ but $y \in Y$. Since $X \subseteq \bar{Y}$ and $Y$ maximally avoids $y$, we have $Y, x \vdash y$ for each $x \in X$ although $Y \nvdash y$. So $Y \not{ }^{c c} X$, and therefore (by $(\mathbb{M})$ ), as $y \in Y, y \nvdash^{\text {cc }} X$. But $X \vdash y$, so this contradicts the assumption that $\vdash$ satisfies $\left(\right.$ Reverse $\left.^{\text {cc }}\right)$.

Thus in view of the equivalence between $\left(\right.$ Reverse $^{c c}$ ) and (Reverse ${ }^{\exists c c}$ ), Thm. 2.1.3 records the success of the ' $\exists$ cc' strategy as applied to (Reverse).

Because the treatment provided by Thm. 2.1.3 will serve as something of a model for our approach to the analogous question for $\sim$ in 3.1 (Problem 1.1.2, that is), we elaborate on some of its aspects here. As for the case of $\sim$, there are two questions, parallelling those raised as (16) and (17) in 1.2, that might be asked to see whether the above proof makes life harder than is necessary in not just using characteristic functions of $\vdash$-closed sets, or just using characteristic functions of maximal avoiders. Do we really need, that is, to begin with the latter and then throw in the characteristic functions of their complements, noting that the latter are consistent valuations? We call the corresponding questions $\left(16^{-}\right)$and $\left(17^{-}\right)$.

$\left(16^{-}\right)$If $\vdash$ is a closure relation determined by some class of valuations closed under ${ }^{-}$, must the class of maximal avoiders relative to $\vdash$ be closed under taking complements?

$\left(17^{-}\right) \quad$ If $\vdash$ is a closure relation determined by some class of valuations closed under ${ }^{-}$, must the class of $\vdash$-closed sets be closed under taking complements? (Equivalently: must $\mathscr{V}_{a t}(\vdash)$ be closed under ${ }^{-}$?)

The answer is in both cases negative, showing that a simple-minded approach along either of the lines canvassed above is not available:

Example 2.1.4. Let $U$ be the 3-element set $\{a, b, c\}$ and let $\mathscr{V}$ contain four valuations: $v_{1}$, with $v_{1}(a)=\mathrm{T}, v_{1}(b)=v_{1}(c)=\mathrm{F} ; v_{2}$, with $v_{2}(b)=\mathrm{T}, v_{2}(a)=$ $v_{2}(c)=\mathrm{F}$; together with $\bar{v}_{1}$ and $\bar{v}_{2}$. It is not hard to see that the closure relation determined by $\mathscr{V}$ is the least closure relation $\vdash$ on $U$ such that $a, b \vdash c$ and $a, c \vdash b$. The $\vdash$-closed sets are $\varnothing,\{a\},\{b\},\{c\}$, and $\{b, c\}$ and $\{a, b, c\}$; the maximal avoiders are $\{b\}$, which maximally avoids $c$ (though not $a$ ), $\{c\},\{a\}$, and $\{b, c\}$.

Consideration of the closure relation $\vdash$ of this Example shows that the answers to $\left(16^{-}\right)$and $\left(17^{-}\right)$are negative. Clearly the class $\mathscr{V}$ which determines $\vdash$ is closed under the operation ${ }^{-}$. (Note: we are not saying that $\mathscr{V}=\mathscr{V} a t(\vdash)$.) But, 
taking $\left(17^{-}\right)$to begin with, the complement $\{a, b\}$ of the $\vdash$-closed $\{c\}$ is not itself $\vdash$-closed. In this case $\{c\}$ is also a maximal avoider, so since its complement is not even $\vdash$-closed it is certainly not a maximal avoider, and we have a negative answer to Question (16-) also. We will have occasion to return to Example 2.1.4 again in 2.5, and some aspects of the closure relation it presents have been chosen with that later application in mind.

\subsection{Disjunctive Combinations}

When is $\mathscr{V}_{a t}(\vdash)$ closed under the operation $\nabla$, for a generalized closure relation $\vdash$ ? We need the following condition on $\vdash$ :

(Left Unions) $\quad X_{0}, X_{1} \vdash Y$ implies $X_{0} \vdash Y$ or $X_{1} \vdash Y$

Theorem 2.2.1. A generalized closure relation $\vdash$ satisfies (Left Unions) iff Vat $(\vdash)$ is closed under $\nabla$.

Proof. 'If': Suppose that $\mathscr{V}_{a t}(\vdash)$ is closed under $\nabla$ and that $X_{0} \not \nvdash Y, X_{1} \not \vdash Y$, for some $X_{0}, X_{1}, Y$. We must show that $X_{0}, X_{1} \not \nvdash Y$. Our supposition gives $v_{0}, v_{1} \in \mathscr{V}_{a t}(\vdash)$ with $v_{0}\left(X_{0}\right)=v_{1}\left(X_{1}\right)=\mathrm{T}, v_{0}(Y)=v_{1}(Y)=\mathrm{F}$, so that $v_{0} \nabla v_{1}\left(X_{0} \cup X_{1}\right)=\mathrm{T}$ while $v_{0} \nabla v_{1}(Y)=\mathrm{F}$; since $v_{0} \nabla v_{1} \in \mathscr{V a t}(\vdash)$, we have $X_{0}, X_{1}$ $\forall Y$.

'Only if': Suppose that $\vdash$ satisfies (Left Unions) and that $v_{0}, v_{1} \in \mathscr{V}_{\text {at }}(\vdash)$ while $v_{0} \nabla v_{1} \notin \mathscr{V}_{a t}(\vdash)$; thus there are $X, Y$ with $X \vdash Y, v_{0} \nabla v_{1}(X)=\mathrm{T}, v_{0} \nabla v_{1}(Y)=\mathrm{F}$. Let $X_{0}=\left\{u \in X \mid v_{0}(u)=\mathrm{T}\right\}, X_{1}=\left\{u \in X ; v_{1}(u)=\mathrm{T}\right\}$. Note that $X=X_{0} \cup X_{1}$ and that $v_{0}(Y)=v_{1}(Y)=\mathrm{F}$. These assignments contradict the fact that $v_{0}, v_{1} \in$ $\mathscr{V}_{\text {at }}(\vdash)$ since, by (Left Unions) and the fact that $X \vdash Y$, we must have either $X_{0}$ $\vdash Y$ or else $X_{1} \vdash Y$.

Turning now to closure relations, our usual practice of superscripting ' $\exists$ 's suggests the condition

(Left Unions ${ }^{\exists}$ ) $\quad X_{0}, X_{1} \vdash^{\exists} Y$ implies $X_{0} \vdash^{\exists} Y$ or $X_{1} \vdash^{\exists} Y$

which is easily seen to be equivalent to the following simplified formulation:

(Simp. Unions) $\quad X_{0}, X_{1} \vdash y$ implies $X_{0} \vdash y$ or $X_{1} \vdash y$.

Theorem 2.2.2. A closure relation $\vdash$ satisfies (Simp. Unions) iff $\mathscr{V}_{a t}(\vdash)$ is closed under $\nabla$.

Proof. The proof parallels that of Theorem 2.2.1, with $y$ playing the role played there by $Y$. 
The 'if' part of the argument here holds for any $\mathscr{V}$ determining $\vdash$, so, bearing in mind the fact that $\mathscr{V}_{a t}(\vdash)$ is one of the classes of valuations which determine $\vdash$, we have:

Corollary 2.2.3. A closure relation $\vdash$ satisfies (Simp. Unions) iff some class of valuations determining $\vdash$ is closed under $\nabla$.

Atypically, then, for the case of $\nabla$, one and the same condition works to delimit the closure relations determined by some class of valuations or other which is closed under the operation, and the closure relation the class of all whose consistent valuations is closed under that operation. There was, in particular, no need to resort to (characteristic functions of) maximal avoiders to obtain the former result (with an attendant restriction to Lindenbaum closure relations). It is interesting to note the comparison here with the Galois-dual connective, $\vee$, for which determination (of consequence relations satisfying the obvious rule-like conditions) by the class of $\vee$-boolean valuation does require consideration of maximal avoiders, since many non- $\vee$-boolean valuations will be amongst $\mathscr{V}_{a l}(\vdash)$ in this case (in view of Prop. 0.2.3 and the fact that conjunctive combinations of $\checkmark$-boolean valuations are not in general $\vee$-boolean). We will see a similar situation for $v_{\mathrm{F}}$ in Thm. 2.5.3.

Though the present case in unusual in not offering distinct conditions for closure of $\mathscr{V}_{a}(\vdash)$ under $\nabla$ and closure of some $\vdash$-determining class under $\nabla$, it is no different from what we saw form 2.1 for $^{-}$in another respect, namely the success for the 'some determining class' result of the general ' $\exists$ cc' strategy, which worked for ${ }^{-}$in delivering (Reverse ${ }^{\exists c c}$ ) from (Reverse), though we conducted the discussion (and in particular Thm. 2.1.3) in terms of the simplified version (Reverse ${ }^{c c}$ ) of the condition thus delivered. When we apply this strategy to (Left Unions), we obtain

$$
\text { (Left Unions }^{\exists \mathrm{cc}} \text { ) } \quad X_{0}, X_{1} \vdash^{\exists} Y \text { implies } X_{0} \vdash^{\mathrm{cc}} Y \text { or } X_{1} \vdash^{\mathrm{cc}} Y
$$

and this condition, just like (Left Unions ${ }^{\exists}$ ) simplifies to (Simp. Unions). So in the present instance, the strategies which in 2.1 yielded non-equivalent conditions on closure relations $-\left(\right.$ Reverse $\left.^{\exists}\right)$ and $\left(\right.$ Reverse $\left.^{\exists c c}\right)$, yield instead conditions which are equivalent.

It is also worth mentioning, $\bar{a}$ propos of the present case, that for a finitary closure relation, the condition we have called (Simp. Unions) is equivalent to the condition (not itself a generalized rule-like condition in the sense of 0.4) that $\vdash$ is 'left-prime', meaning that:

$$
X \vdash z \text { implies } x \vdash z \text { for some } x \in X \text {, provided } X \neq \varnothing \text {. }
$$

The fact that for any language $U$ closed under at least the connective $\vee$ the consequence relation on $U$ determined by the class of all $\vee$-boolean valuations (for 
$U$ ) enjoys this left-primeness relation follows from the finitariness of that relation ('compactness theorem for fragments of classical sentential logic') together with Prop. 0.3 .2 (ii). In fact in this case we can omit the proviso that $X \neq \varnothing$ since we never have $\varnothing \vdash z$ for this consequence relation (as $v_{\mathrm{F}} \in \mathscr{V}_{\text {at }}(\vdash)$ ): see Thm. 2.5.3 and the discussion preceding it).

\subsection{Conjunctive Combinations}

The appropriate condition on generalized closure relations $\vdash$ is the following: (Right Unions) $\quad X \vdash Y_{0}, Y_{1}$ implies $X \vdash Y_{0}$ or $X \vdash Y_{1}$

Theorem 2.3.1. A generalized closure relation $\vdash$ satisfies (Right Unions) iff $\mathscr{V}_{\text {al }}(\vdash)$ is closed under $\triangle$.

Proof. A straightforward adaptation of the proof of Thm. 2.2.1.

Turning to closure relations, we note that the result of superscripting ' $\exists$ 's to every ' $\vdash$ ' in the condition (Right Unions) yields someting - call it (Right Unons ${ }^{\exists}$ ) - automatically satisfied by every closure relation; and of course we already know (Coro. 0.2.4 (i)) that for all closure relations $\vdash$, Vat $(\vdash)$ is closed under binary (indeed arbitrary) conjunctive combination of valuations. So the answer to the question 'When is a closure relation determined by some class of valuations closed under $\triangle$ ?' is: Always. Thus, as with $\nabla$ (see Coro. 2.2.3 and the remark immediately following), the distinction between having $\mathscr{V}_{a}(\vdash)$ closed under the operation and having some determining class or other thus closed is nullified in the present context.

What becomes of what we have been calling the ' $\exists c c$ ' strategy in this case? As applied to (Right Unions), it delivers the condition:

(Right Unions ${ }^{\exists c c}$ ) $X \vdash^{\exists} Y_{0}, Y_{1}$ implies $X \vdash^{\mathrm{cc}} Y_{0}$ or $X \vdash^{\mathrm{cc}} Y_{1}$

This condition differs from that alluded to above as (Right Unions ${ }^{\exists}$ ) by replacing the consequent's $\vdash^{\exists}$-statements by the (if anything) weaker corresponding $\vdash^{\text {cc }}$ -statements, so it too is trivially satisfied by all closure relations. So we must rule the ' $\exists \mathrm{cc}$ ' strategy successful for the present case also, albeit in a less interesting way than for the case of $\nabla$.

We have a verdict similar to that returned above for closure of determining classes under $\triangle$ for the case of closure relations determined by classes of valuations closed under (i.e., in the present instance, containing) $v_{\mathrm{T}}$ : every closure relation is so determined, by Coro. 0.2.4 (ii). We may as well treat here also the generalized closure relations determined by classes of valuations containing $v_{\mathrm{T}}$ :

Theorem 2.3.2. For a generalized closure relation $\vdash$ on $U, v_{\mathrm{T}} \in \mathscr{V}_{a d}(\vdash)$ iff 
$U \nvdash \varnothing$.

The condition here invoked $(U \nvdash \varnothing)$ is a generalized rule-like condition in the sense of 0.4 , being a case of the general schema (12) of that discussion, in which $n=0$. This corresponds to the fact that $v_{\mathrm{T}}$ is a 0 -place operation on valuations, just as the Galois-duals of the binary connectives require conditions in which $n=2$ (and for negative objects, in 2.1, we had $n=1$ : a rule-like condition proper-namely (Reverse) - in this case). We remark also that the condition employed in Thm. 2.3.2 is equivalent to the denial that $\vdash$ is 'left-assertive' in the sense of [24], p.39.

\subsection{Implicative Combinations}

We begin as usual with the case of generalized closure relations. The appropriate condition on $\vdash$ is for closure of determining classes of valuations under implicative combination $(\Rightarrow)$ of valuations is:

(Impl) $X_{0}, X_{1} \vdash Y$ implies $Y \vdash X_{0}$ or $X_{1} \vdash Y$

Theorem 2.4.1. A generalized closure relation $\vdash$ satisfies (Impl) iff $\mathscr{V}_{a}(\vdash)$ is closed under the operation $\Rightarrow$.

Proof. 'If': Suppose that $\mathscr{V}_{a t}(\vdash)$ is closed under $\Rightarrow$ and (1) $Y \not \nvdash X_{0}$, (2) $X_{1} \not \vdash$ $Y$. (1) and (2) give respectively $v_{1}, v_{2} \in \mathscr{V}_{a t}(\vdash)$ with $v_{1}(Y)=\mathrm{T}, v_{1}\left(X_{0}\right)=\mathrm{F}$, $v_{2}\left(X_{1}\right)=\mathrm{T}, v_{2}(Y)=\mathrm{F}$. Thus $v_{1} \Rightarrow v_{2}\left(X_{0}\right)=v_{1} \Rightarrow v_{2}\left(X_{1}\right)=\mathrm{T}$ while $v_{1} \Rightarrow v_{2}(Y)=\mathrm{F}$. So, since $v_{1} \Rightarrow v_{2} \in \mathscr{V}_{\text {at }}(\vdash), X_{0}, X_{1} \nvdash Y$.

'Only if': Suppose that $\vdash$ satisfies (Impl) and-for a contradiction - that we have $v_{1}, v_{2} \in \mathscr{V}_{a t}(\vdash)$ but $v_{1} \Rightarrow v_{2} \notin \mathscr{V}_{a t}(\vdash)$. Since $v_{1} \Rightarrow v_{2} \notin \mathscr{V}_{a t}(\vdash)$, there exist $X, Y$ with $X \vdash Y, v_{1} \Rightarrow v_{2}(X)=\mathrm{T}, v_{1} \Rightarrow v_{2}(Y)=\mathrm{F}$. Since $v_{1} \Rightarrow v_{2}(X)=\mathrm{T}, X$ is the union of (not necessarily disjoint) sets $X_{0}=\left\{u \in X \mid v_{1}(u)=\mathrm{F}\right\}$ and $X_{1}=$ $\left\{u \in X \mid v_{2}(u)=\mathrm{T}\right\}$, and thus, as $X \vdash Y$, we may appeal to (Impl) to conclude that either $Y \vdash X_{0}$ or $X_{1} \vdash Y$. Since $v_{1} \Rightarrow v_{2}(Y)=\mathrm{F}, v_{1}(Y)=\mathrm{T}$ and $v_{2}(Y)=\mathrm{F}$. But as $v_{1}(Y)=\mathrm{T}$ and $v_{1}\left(X_{0}\right)=\mathrm{F}$, we cannot have $Y \vdash X_{0}$, and as $v_{2}\left(X_{1}\right)=\mathrm{T}$ and $v_{2}(Y)=\mathrm{F}$, we cannot have $X_{1} \vdash Y$ either.

For closure relations, we modify (Impl) in a now predictable way to obtain a condition on $\vdash$ necessary and sufficient for $\mathscr{V}_{a d}(\vdash)$ to be closed under $\Rightarrow$ :

$\left(\operatorname{Impl} l^{\exists \exists}\right) \quad X_{0}, X_{1} \vdash^{\exists} Y$ implies $Y \vdash^{\exists} X_{0}$ or $X_{1} \vdash^{\exists} Y$

This condition can be simplified to the equivalent:

$\left(\operatorname{Impl} l^{\exists}\right) \quad X_{0}, X_{1} \vdash y$ implies $y \vdash^{\exists} X_{0}$ or $X_{1} \vdash y$

An argument directly parallelling the proof of Thm. 1.2.4 then establishes: 
Theorem 2.4.2. A closure relation $\vdash$ satisfies $\left(\operatorname{Impl} l^{\exists}\right)$ iff $\mathscr{V}_{a t}(\vdash)$ is closed under $\Rightarrow$.

To answer the more general question of when a closure relation is determined by some $\Rightarrow$-closed class or other, we make a familiar adjustment to the superscripting in $\left(\operatorname{Impl} l^{\exists}\right)$ :

$\left(\operatorname{Impl} l^{\mathrm{cc}}\right) \quad X_{0}, X_{1} \vdash y$ implies $y \vdash^{\mathrm{cc}} X_{0}$ or $X_{1} \vdash y$

The direct result of implementing the ' $\exists$ cc' strategy would cnvert (Impl) to $\left(\operatorname{Impl} l^{\exists \mathrm{cc}}\right) \quad X_{0}, X_{1} \vdash^{\exists} Y$ implies $Y \vdash^{\mathrm{cc}} X_{0}$ or $X_{1} \vdash^{\mathrm{cc}} Y$

We invite the reader to check that a closure relation satisfies ( $\left(\mathrm{Impl}^{\mathrm{cc}}\right.$ ) if and only if it satisfies ( $\mathrm{Impl}^{\exists \mathrm{cc}}$ ). In view of this equivalence, Thm. 2.4.4 below shows that the ' $\exists \mathrm{cc}$ ' strategy is again successful. (Cases for which the strategy does not issue in success have been postponed for treatment in 2.5.)

To show that if $\vdash$ satisfies ( $\left(\mathrm{Impl}^{\mathrm{cc}}\right.$ ), we can find a determining class of valuations closed under $\Rightarrow$, we record first a preliminary observation:

Lemma 2.4.3. If $W$ maximally avoids $w$, relative to a closure relation $\vdash$ satisfying $\left(\operatorname{Impl} l^{\mathrm{cc}}\right)$, and $x \notin W$, then $w \vdash x$.

Proof. Under the hypothesis of the Lemma, if $x \notin W$ then $W, x \vdash w$ so invoking ( $\mathrm{Impl}^{\mathrm{cc}}$ ) we get: either $w \vdash x$ (equivalently, $w \vdash{ }^{\mathrm{cc}} x$ ) or $W \vdash w$. But $W$ $\forall-w$, since $w$ was chosen as something maximally avoided by $W$.

One consequence of Lemma 2.4.3, not directly exploited below but worth making explicit, is that if $W$ is a maximal avoider and $x \notin W$, then $W$ maximally avoids $x$ : in other words (0.2) all maximal avoiders are absolute maximal avoiders.

For our proof, we will be taking valuations as the characteristic functions of sets $v_{W} \Rightarrow v_{Z}=v_{\bar{W} \cup Z}$. Obviously, starting with maximal avoiders and closing under this operation can lead us outside the class - as when $W=Z$, in which case we get $U$ as the result (with characteristic function $v_{\mathrm{T}}$ ): not a maximal avoider. We can succeed if we broaden our horizons just enough to let in this one additional case, however. To that end, we define a set $W$ to be full (relative to $\vdash$ ) if either $W=U$ or else $W$ is a maximal avoider (relative to $\vdash$ ). Since any Lindenbaum closure relation $\vdash$ is determined by the class of valuations which are characteristic functions of maximal avoiders (relative to $\vdash$ ), we have by Coro. 0.2.4 (iii) that $\vdash$ is also determined by the union of this class with $\left\{v_{T}\right\}$, and hence by the class $\left\{v_{W} \mid W\right.$ is full, relative to $\left.\vdash\right\}$.

Theorem 2.4.4. A Lindenbaum closure relation $\vdash$ satisfies $\left(\operatorname{Impl} l^{\mathrm{cc}}\right)$ iff some class of valuations which determines $\vdash$ is closed under $\Rightarrow$. 
Proof. 'If'. We leave the reader to verify (substantially along the same lines as in the corresponding proof of Thm. 2.4.1) that any closure relation determined by a class of valuations closed under $\Rightarrow$ must satisfy $\left(\mathrm{Impl}^{\mathrm{cc}}\right)$.

'Only if'. If we have $\vdash$ meeting the conditions of the Theorem, then we take the determing class $\mathscr{V}$ to be comprise the characteristic functions of sets which are full relative to $\vdash$. We must show that these are closed under the operation $\Rightarrow$ where $v_{W_{0}} \Rightarrow v_{W_{1}}=v_{\bar{W}_{0} \cup W_{1}}$. So suppose that $W_{0}$ and $W_{1}$ are full sets, with a view to showing that $\bar{W}_{0} \cup W_{1}$ is in that case full. If either $W_{0}$ or $W_{1}$ is $U$ then $\bar{W}_{0} \cup W_{1}=W_{1}$, so we need only show, assuming $W_{0}, W_{1}$, full that $\bar{W}_{0} \cup W_{1}$ is full for the case in which each of $W_{0}, W_{1}$, is a maximal avoider, avoiding respectively (say) $w_{0}, w_{1}$. Further, if $W_{0} \subseteq W_{1}$, then $\bar{W}_{0} \cup W_{1}=U$ and is therefore full, so it will suffice to consider the case in which for some $x, x \in W_{0}, x \notin W_{1}$. We claim that in this case, $\bar{W}_{0} \cup W_{1}$ maximally avoids $w_{1}$. So we must show

(1) $\bar{W}_{0} \cup W_{1} \nvdash w_{1}$ and

(2) For all $u \notin \bar{W}_{0} \cup W_{1}: \bar{W}_{0} \cup W_{1}, u \vdash w_{1}$

(2) is easily established: if $u \notin \bar{W}_{0} \cup W_{1}$ then $u \notin W_{1}$, so $W_{1}, u \vdash w_{1}$, and so $\bar{W}_{0} \cup W_{1}$, $u \vdash w_{1}$. We turn to establishing (1). Suppose, for a contradiction, that $\bar{W}_{0}, W_{1} \vdash$ $w_{1}$. Recall the element $x$ which belonged to $W_{0}$ but not to $W_{1}$; by Lemma 2.4.3, $w_{1} \vdash x$, so (by $\left.(\mathbb{T})\right), \bar{W}_{0}, W_{1} \vdash x$. By the condition $\left(\mathrm{Impl}^{\mathrm{cc}}\right.$ ), then, either $x \vdash^{\mathrm{cc}} \bar{W}_{0}$ or $W_{1} \vdash x$. Since $W_{1}$ is a maximal avoider and $x \notin W_{1}$, it is the first disjunct here which holds: $x \vdash^{\text {cc }} \bar{W}_{0}$. But $x \in W_{0}$, so this implies (by (M)) $W_{0} \vdash{ }^{\text {cc }} \bar{W}_{0}$, contradicting Prop. 0.5.1 (ii).

\subsection{Some Further Cases}

We give a brief treatment of operations Galois-dual to the two remaining boolean connectives listed in 0.2 , namely exclusive disjunction (sentence connective: $\vee$; Galois-dual: $\underline{\nabla}$ ) and the Falsum constant (sentence connective $\perp$; Galois dual $v_{\mathrm{F}}$ ). Then we go on to consider the Galois-dual of a connective ('nor') not listed earlier, which will lead into a discussion of requiring that a determining class of valuations be closed under Galois-duals of several boolean sentence connectives at once.

We begin with exclusive disjunctive combinations. For generalized closure relations we have the condition

(Excl) $\quad X_{0}, X_{1} \vdash Y_{0}, Y_{1}$ implies $X_{0}, Y_{1} \vdash X_{1}, Y_{0}$ or $X_{1}, Y_{1} \vdash X_{0}, Y_{0}$ and for closue relations, we append ' $\exists$ ' superscripts:

$\left(\operatorname{Excl}^{\exists}\right) \quad X_{0}, X_{1} \vdash^{\exists} Y_{0}, Y_{1}$ implies $X_{0}, Y_{1} \vdash^{\exists} X_{1}, Y_{0}$ or $X_{1}, Y_{1} \vdash^{\exists} X_{0}, Y_{0}$

We omit proof of the following, since it involves no novelties: 
Theorem 2.5.1. A generalized closure relation $\vdash$ satisfies (Excl) iff $\mathscr{V}_{a t}(\vdash)$ is closed under the operation $\underline{\nabla}$.

Linkewise in the case of

Theorem 2.5.2. A closure relation $\vdash$ satisfies $\left(\right.$ Excl $\left.^{\exists}\right)$ iff $\mathscr{V}_{a \ell}(\vdash)$ is closed under the operation $\underline{\nabla}$.

An analogous result for a condition related to $\left(\operatorname{Excl}^{\exists}\right)$ as (Flip-around $\left.{ }^{\exists c c}\right)$ is related to (Flip-around ${ }^{\exists}$ ):

(Excl $\left.{ }^{\exists c c}\right) X_{0}, X_{1} \vdash^{\exists} Y_{0}, Y_{1}$ implies $X_{0}, Y_{1} \vdash^{c c} X_{1}, Y_{0}$ or $X_{1}, Y_{1} \vdash^{c c} X_{0}, Y_{0}$

encounters the same difficulties as were foreshadowed for (Flip-around ${ }^{\text {cc }}$ ) and its equivalent, (Flip-around ${ }^{\exists c c}$ ) in 1.2. We will return to those difficulties at the toward the end of the present subsection and deal with them more fully for the latter condition(s) in $\S 3$; a similar approach can be followed for obtaining a generalized rule-like condition both necessary and sufficient for any Lindenbaum closure relation satisfying it to be determined by some class of valuations closed under $\nabla$, as we shall note at the end of the present discussion. (It is easy to see that the 'necessity' part of this desideratum holds for $\left(\operatorname{Excl}^{\exists \mathrm{cc}}\right)$ : any closure relation determined by a $\underline{\nabla}$-closed class of valuations must satisfy this condition.)

The remaining case listed in 0.2 arises naturally from consideration of classes of valuations closed under exclusive disjunctive combination: the presence of the valuation $v_{\mathrm{F}}$ assigning $\mathrm{F}$ to every element of the given underlying set $U$. Since for any valuation $v, v \underline{\nabla} v=v_{\mathrm{F}}$, a non-empty class closed under $\underline{\nabla}$ must contain $v_{\mathrm{F}}$. (A similar point about $\sim$ and $v_{\mathrm{T}}$ was made in 1.1.) So let us address the question of determination of (generalized) closure relations by classes of valuations containing $v_{\mathrm{F}}$. (The case of $v_{T}$ was treated in 2.3.)

If we are thinking of $U$ as a language, and $\vdash$ as a consequence relation on $U$, then for $v_{\mathrm{F}}$ to be consistent with $\vdash, \vdash$ must be 'atheorematic': for no $y \in U$ do we have $\vdash y$. Whether or not the particular choice of $\vdash$ and $U$ makes this terminology appropriate, the condition concerned, which we can write more succinctly as ' $\forall{ }^{\exists} U$ ', is easily seen to be both necessary and sufficient for determination by a class of valuations containing $v_{\mathrm{F}}$. We state this in part (i) of Thm. 2.5.3; part (ii) deals with generalized closure relations by omitting the superscript ' $\exists$ '. The proofs are straightforward; for part (ii) the standing assumption that $U \neq \varnothing$ needs to be exploited; the condition employed for this part is equivalent to the denial that $\vdash$ is what Segerberg calls 'right-assertive' at p.39 of [24].

Theorem 2.5.3. (i) For any closure relation $\vdash$ on $U$, the following are equivalent: (1) $\nvdash^{\exists} U$, (2) $v_{\mathrm{F}} \in \mathscr{V}_{a t}(\vdash),(3) \vdash$ is determined by some class of valuations containing $v_{\mathrm{F}}$. 
(ii) For any generalized closure relation $\vdash$ on $U, v_{\mathrm{F}} \in \mathscr{V}_{\text {at }}(\vdash)$ iff $\nvdash U$.

The equivalence of (2) with (3) under (i) here is analogous to the result for $\nabla$ given as Coro. 2.2.3 above: no distinction between the 'some determining class' and the ' $\mathscr{V}_{a l}(\vdash)$ 'cases. Conspicuously missing is any reference to the condition on a closure relation $\vdash$ to the effect that $\nvdash^{\mathrm{cc}} U$, and we make a few remarks about what such a condition would imply.

Directly unpacking the definition of $\vdash^{\mathrm{cc}}$, we can see that the condition just mentioned amounts to the claim that there is some (super) set $X^{\prime}$ (of $\varnothing$ ) and some element $z$, with (1) $X^{\prime}, u \vdash z$ for each $u \in U$, while (2) $X^{\prime} \forall u$. (1) and (2) together imply that $X^{\prime}$ must be $\varnothing$, so what our condition tells us is more something about what's in $U$ than about what's in those $\mathscr{V}$ determining $\vdash$ :

namely, that $U$ contains some element ( $z$ above) which, although not in the closure of the empty set, is in the closure of every non-empty set. Let us temporarily baptize any element of $U$ with the above properties a mere follower (relative to $\vdash)$. Alternative definiton: a mere follower is an element which $\varnothing$ maximally avoids. (From the specifically logical perspective, this would be a formula which, altough unprovable, follows from - is a consequence of-every formula.) If $\vdash$ is a Lindenbaum closure relation, this does in fact tell us a great deal about determining classes of valuations, but we first approach the matter anew, using what Prop. 0.5 .1 (vi) says about $\vdash^{\mathrm{cc}}$ for the case in which $\vdash$ is a Lindenbaum closure relation. That result, which gives a general equivalent to $X \vdash^{\text {cc }} Y$ in terms of determining classes of valuations, has the following form for the case in which $X=\varnothing$ :

$$
\begin{aligned}
& \vdash^{\mathrm{cc}} Y \text { iff there is some } \mathscr{V} \text { determining } \vdash \text { such that for all } \\
& v \in \mathscr{V} u(y)=\mathrm{T} \text { for some } y \in Y .
\end{aligned}
$$

So taking $Y=U$ and negating both sides, we get

$$
\begin{aligned}
& \nvdash^{\mathrm{cc}} U \text { iff for every } \mathscr{V} \text { determining } \vdash \text {, there exists } v \in \mathscr{V} \text { with } \\
& v(u)=\mathrm{F} \text { for all } u \in U .
\end{aligned}
$$

In other words, we have:

Proposition 2.5.4. For any Lindenbaum closure relation $\vdash$ on a set $U$, we have $\forall{ }^{\mathrm{cc}} U$ iff every class of valuations which determines $\vdash$ contains $v_{\mathrm{F}}$.

To relate this characterization of closure relations such that $\forall{ }^{c c} U$ to that arrived at earlier in terms of the existence of a 'mere follower' in $U$, note that, since if $z$ is such an element, we have $\forall z$ so any determining class of valuations must contain a $v$ with $v(z)=\mathrm{F}$; but also, since $u \vdash z$ for all $u \in U$, for every $u$ we have $v(u)=\mathrm{T}$ : in other words, $v=v_{\mathrm{F}}$. Conversely - and here we need the hypothesis that $\vdash$ is a Lindenbaum closure relation - if every class of valuations determining $\vdash$ contains $v_{\mathrm{F}}$, then this is so for the class of characteristic functions of maximal 
avoiders relative to $\vdash$ (Thm. 0.2.2). Now $v_{\mathrm{F}}$ is the characteristic function of $\varnothing$, so this must be a maximal avoider, and any element maximally avoided by $\varnothing$ is a mere follower.

It was mentioned above that there are difficulties for finding a generalized rule-like condition on closure relations necessary and sufficient for determination by some $\underline{\nabla}$-closed class of valuations which are analogous to those we shall be examining in the case of $\sim$ in $\S 3$. These are operations Galois-dual to connectives which are associated over the class of their respectively boolean valuations with 'alternating' truth-functions in Post's taxonomy ([21]), and we want to give some account of what those difficulties are while at the same time indicating that they are not peculiar to the alternating cases. For this reason, we deal here with a new truth-function not covered in our listing in 0.2 ; we choose an example which conveiently allows the discussion to move into the area of functional completeness (and more generally, functional composition). Some additional truth-functions and the corresponding Galois-dual operations will enter the discussion later.

The new case we consider is that of 'joint denial', also called 'nor'; our notation for this (binary) connective will be ' $\bar{\nabla}$ ' and for the Galois-dual operation, $\bar{\nabla}$. That is, we define a valuation $v$ (for $U$ ) to be $\bar{V}$-boolean just in case for all $x, y \in U$ :

$$
v(x \bar{\vee} y)=\mathrm{T} \text { iff } v(x)=v(y)=\mathrm{F}
$$

and for valuations $v_{1}, v_{2}$, we define $v_{1} \bar{\nabla} v_{2}$ by setting:

$$
v_{1} \bar{\nabla} v_{2}(u)=\mathrm{T} \text { iff } v_{1}(u)=v_{2}(u)=\mathrm{F}
$$

for all $u \in U$.

Letting (Nor) and $\left(\right.$ Nor $^{\exists}$ ) be the conditions on, respectively generalized closure relations and closure relations $\vdash$ :

$$
\begin{aligned}
& \text { (Nor) } \quad X \vdash Y_{0}, Y_{1} \text { implies } Y_{0} \vdash X \text { or } Y_{1} \vdash X \\
& \text { (Nor } \left.{ }^{\exists}\right) \quad X \vdash^{\exists} Y_{0}, Y_{1} \text { implies } Y_{0} \vdash^{\exists} X \text { or } Y_{1} \vdash^{\exists} X
\end{aligned}
$$

we have, by the methods of our earlier proofs:

Proposition 2.5.5. $(i)$ For a generalized closure relation $\vdash$, Tat $(\vdash)$ is closed under $\bar{\nabla}$ iff $\vdash$ satisfies (Nor).

(ii) For a closure relation $\vdash$, Vat $(\vdash)$ is closed under $\bar{\nabla}$ iff $\vdash$ satisfies $\left(N o r^{\exists}\right)$.

The obvious modification to (Nor ${ }^{\exists}$ ) to obtain a condition apt for closure relations satisfying it to be precisely those determined by some class of valuations or other which is closed under $\bar{\nabla}$ would be to change the ' $\exists$ 's on the consequent to 'cc's: 
(Nor ${ }^{\exists c c}$ ) $X \vdash^{\exists} Y_{0}, Y_{1}$ implies $Y_{0} \vdash^{c c} X$ or $Y_{1} \vdash^{c c} X$

But while satisfying this condition is certainly necessary for $\vdash$ to be determined by some $\bar{\nabla}$-closed class of valuations, there is a difficulty about showing its sufficiency which precisely parallels that to be raised for (Flip-around ${ }^{\text {cc }}$ ) - or equivalently for the condition (Flip-around ${ }^{\exists c c}$ ) on which (Nor ${ }^{\exists c c}$ ) is modelled in Example 3.2.1. Roughly, and in the simplest possible case the difficulty is as follows. (Exactly why this constitutes a difficulty for the strategy of $\S 3$ will become clear in that section.) While the condition tells us that applying $\bar{\nabla}$ to pairs, $v_{1}, v_{2}$, of characteristic functions of maximal avoiders (relative to closure relations which satisfy it) yields a valuation $v_{1} \bar{\nabla} v_{2}$ consistent with $\vdash$, we want the result of applying it to the pair consisting of such a $v_{1} \bar{\nabla} v_{2}$ and $v_{3}$ (say), the characteristic function of another maximal avoider, $\left(v_{1} \bar{\nabla} v_{2}\right) \bar{\nabla} v_{3}$, also to be consistent, and nothing in (Nor ${ }^{\exists c c}$ ) guarantees this. (The above claim about what the condition "tells us" alludes to a derivation like that in the proof of Prop. 3.1.3 of the condition

$$
W \bar{\cup} W^{\prime} \vdash{ }^{\exists} \overline{W \bar{\cup} W^{\prime}} \text { implies } W \vdash{ }^{\mathrm{cc}} \bar{W} \text { or } W^{\prime} \vdash^{\mathrm{cc}} \bar{W}^{\prime}
$$

from (Nor ${ }^{\exists c c}$ ), where $W \bar{\cup} W^{\prime}=\overline{W \cup W^{\prime}}$. (Thus $v_{X} \bar{\nabla} v_{Y}=v_{X \bar{\cup} Y}$. ))

It would not, incidentally, be appropriate to respond to the above difficulties for $\left(\right.$ Nor $^{\exists c c}$ ) by attempting a generalization along the lines of:

$$
X \vdash^{\exists} Y_{0}, Y_{1}, \ldots, Y_{n} \text { implies } Y_{0} \vdash^{\mathrm{cc}} X \text { or } Y_{1} \vdash^{\mathrm{cc}} X \text { or } \ldots \text { or } Y_{n} \vdash^{\mathrm{cc}} X
$$

since a closure relation determined by some $\bar{\nabla}$-closed class of valuations need not satisfy this new condition. For a given $n$, what this condition requires is rather that when $v_{1}, \ldots, v_{n}$ lie within a determining class of valuations, so does $\bar{v}_{1} \nabla \ldots \nabla v_{n}$, which is not in general the same as $\left.\left.\left(\left(\ldots\left(v_{1} \bar{\nabla} v_{2}\right) \bar{\nabla} v_{3}\right) \bar{\nabla} \ldots\right) \bar{\nabla} v_{n-1}\right) \bar{\nabla} v_{n}\right)$, or as any other iterated $\bar{\nabla}$-combination of the $v_{i}$. (Recall that $\bar{\nabla}$ is not associative.)

We can show that not only do we have the above difficulty when it comes to showing that satisfying ( Nor $^{\exists c c}$ ) is sufficient for being determined by some class of valuations closed under $\bar{\nabla}$, but, more conclusively, that this condition is not sufficient for being so determined. (By contrast, the corresponding question for (Flip-around) will be left open in $\S 3$.) To do so, being by noting that the Galoisdual sentence connective, $\bar{V}$, or more properly, the truth-function $f_{\bar{V}}$ associated therewith over any class of $\bar{V}$-boolean valuations ( $c f$. 0.2 ), enjoys a property of weak functional completeness, in the sense that every non-zero-place truthfunction is obtainable from $f_{\mathrm{V}}$ by (projection and) functional composition. This is also true, of course, of the pair $\left\{f_{\neg}, f_{\vee}\right\}$. (Likewise with $\left\{f_{\neg}, f_{\wedge}\right\},\left\{f_{\neg}, f_{\rightarrow}\right\}$; the intended contrast - for which, see [14] - is with strong functional completeness, the analogous property without exemption of the zero-place truth-functions. The pair $\left\{f_{\perp}, f_{\rightarrow}\right\}=\left\{\mathrm{F}, f_{\rightarrow}\right\}$ enjoys this latter property.) Galois-dually, from $\{, \nabla\}$ we can define by composition the operation $\bar{\nabla}$, from which in turn ${ }^{-}, \nabla$, and all the other non-zero-place operations dual to truth-functions, are definable. Thus in 
particular, any class of valuations closed under $\bar{\nabla}$ must be closed under disjunctive combinations and under formation of negative objects. Now, recalling he condition $\left(\right.$ Reverse $\left.{ }^{\mathrm{cc}}\right)$

$$
X \vdash y \text { implies } y \vdash^{c c} X
$$

from 2.1, observe (by a routine deduction of the one condition from the other): $\left(\right.$ Nor $\left.^{\exists \mathrm{cc}}\right)$.

Proposition 2.5.6. Any closure relation satisfying (Reverse ${ }^{\mathrm{cc}}$ ) satisfies

Corollary 2.5.7. Satisfaction of $\left(N o r^{\exists c c}\right)$ by a closure relation is not sufficient for the existence of a determining class of valuations closed under $\bar{\nabla}$.

Proof. The closure relation $\vdash$ described in Example 2.1.4 satisfies (Reverse $^{\mathrm{cc}}$ ), and therefore, by Prop. 2.5.6, (Nor ${ }^{\exists c c}$ ), since $\vdash$ is determined by a class of valuations closed under ${ }^{-}$, namely the class $\mathscr{V}$ exhibited in that Example (by Thm. 2.1.3). But $\vdash$ is not determined by any class of valuations closed under $\nabla$, since the following is a counterinstance to (Simp. Unions), which Coro. 2.2.3 asserts is a condition satisfied by all closure relations so determined: $a, b \vdash c ; a \nvdash c ; b \not \vdash c$. Since any class of valuations closed under $\bar{\nabla}$ is closed under $\nabla, \vdash$ is not determined by a class of valuations closed under $\bar{\nabla}$, even though $\vdash$ satisfies (Nor ${ }^{\exists c c}$ ).

Implicit in the above discussion is a solution to the problem of finding generalized rule-like conditons on closure relations which are not only necessary but-unlike ( Nor $^{\exists c c}$ ) - also sufficient for a closure relation to be determined by some class of valuations closed under $\bar{\nabla}$, but care is needed in making it explicit. In particular, we wish to draw attention to the fallaciousness of the following form of inference:

(i) A certain condition $\mathbf{C}_{1}$ is satisfied by precisely those closure relations (or perhaps: those Lindenbaum closue relations) determined by some class of valuations closed under an operation $\widehat{\#}_{1}$ (dual to boolean sentence connective $\#_{1}$ : we use the notation of 0.3 ).

(ii) Condition $\mathbf{C}_{2}$ is satisfied by precisely those (Lindenbaum) closure relations determined by some class of valuations closure under $\widehat{\#}_{2}$.

\section{Therefore:}

(iii) A (Lindenbaum) closure relation is determined by some class of valuations closed under both $\widehat{\#}_{1}$ and $\widehat{\#}_{2}$ iff it satisfies $\mathbf{C}_{1}$ and $\mathbf{C}_{2}$.

An inference of the above form but having 'determined by some class of valuations closed under' replaced by 'with $\mathscr{V}_{a}(\vdash)$ closed under' would be perfectly correct. Obviously if $\vdash$ satisfies conditions $\mathbf{C}_{1}$ and $\mathbf{C}_{2}$ guaranteeing respectively that $\mathscr{V}_{a t}(\vdash)$ is closed under $\#_{1}$, and that $\mathscr{V}_{a}(\vdash)$ is closed under $\mathbb{\#}_{2}$, then $\mathscr{V}_{\text {at }}(\vdash)$ must be closed under $\widehat{\#}_{1}$ and $\widehat{\#}_{2}$. (Thus there is no analogous difficulty in the case of generalized closure relations, either.) As formulated, however, the 
inference speaks only of the existence of some determining class appropriately closed. So all that follows for $\vdash$ satisfying $\mathbf{C}_{1}$ and $\mathbf{C}_{2}$ as in (i) and (ii) is that $\vdash$ is determined by some class of valuations closed under $\widehat{\#}_{1}$ and also determined by some (not necessarily the same) class of valuations closed under $\widehat{\#}_{2}$; not, as (iii) would have it, by some class of valautions closed under both $\widehat{\#}_{1}$ and $\widehat{\#}_{2}$.

We can re-utilize Example 2.1.3, as in the proof of Coro. 2.5.7 above, to illustrate the fallacy here described in abstract terms. Let $\mathbf{C}_{1}$ be some condition satisfied by all closure relations, such as the conditon $(\mathbb{R})$ from 0.1 , and let $\mathbf{C}_{2}$ be $\left(\right.$ Reverse ${ }^{\text {cc }}$ ). For $\mathbf{C}_{1}$ we choose as our $\widehat{\#}_{1}$ to verify (i), the operation $\triangle$, which is then true since (as remarked in 2.3) every closure relation is determined by some $\triangle$-closed class of valuations. For $\mathbf{C}_{2}$ we choose as $\widehat{\#}_{2}$ to verify (ii), the operation , the justification in this case being provided by Theorem 2.1.3. Since the latter result is restricted to Lindenbaum closure relations, the version of '(i)-(ii)- $\therefore$ (iii)' we are considering here has 'Lindenbaum' in premisses (i), (ii), and in conclusion (iii). We need also a particular closure relation in mind for (i) and (ii), and for this we choose the relation $\vdash$ of Example 2.1.3. The conclusion (iii) would then imply that this closure relation is determined by a class of valuations closed under ${ }^{-}$and $\triangle$. But any class of valuations closed under these two (Galois-dually weakly functionally complete) operations is closed under $\nabla$, which we saw in the proof of Coro. 5.1 .7 is not the case for the present choice of $\vdash$. (The relevant equation here is of course: $v_{1} \nabla v_{2}={\overline{\bar{v}_{1} \triangle \bar{v}_{2}}}_{\text {.) }}$

More specifically, what goes wrong with the case just considered is that the chosen $\vdash$ is determined by a class of valuations closed under $\triangle$ in virtue of the fact that we may take the class of characteristic functions of $\vdash$-closed sets (alias $\mathscr{V}_{a t}(\vdash)$ ) as such a class, whereas $\vdash$ is determined by a class closed under ${ }^{-}$in virtue of the fact that we take the class of characteristic functions of maximal avoiders and their complements as a determining class. Although the latter class of valuations is included in $\mathscr{V}_{a}(\vdash)$, so that any conjunctive combination of its elements is also in $\mathscr{V}_{a t}(\vdash)$, there is no reason for such conjunctive combinations to lie whithin that determining class itself. (See the discussion surrounding Example 2.1.4, and the negative answer provided thereby to Question (17 ${ }^{-}$.)

Incidentally, another way of illustrating the fallaciousness of the above instance of the argument pattern (i)-(ii) $\therefore$ (iii) with reference to the same closure relation $\vdash$ as figured in our discussion would be as follows. If $\vdash$ were determined by a class of valuations closed under $\triangle$ and $^{-}$then it would be closed under $\sim$. But any closure relation determined by a class of valuations closed under matching satisfies (Flip-around ${ }^{c c}$ ), by $\mathrm{Thm} .1 .2 .6$, so that all its maximal avoiders are absolute, by Coro. 1.2.9. Yet for the case of $\vdash$ this is not so: as mentioned under Example 2.1.4, $\{b\}$ maximally avoids $c$ without maximally avoiding $a$.

These cautionary notes having been sounded, we can return to the case of determination of a closure relation - or rather, a Lindenbaum closure relation by some class of valuations closed under $\bar{\nabla}$. (The needed caution is manifested in 
the two-stage definition of $\mathscr{V}$ in the proof.)

Theorem 2.5.8. A Lindenbaum closure relation is determined by some class of valuations closed under $\bar{\nabla}$ iff it satisfies (Simp. Unions) and (Reverse ${ }^{\mathrm{cc}}$ ).

Proof. 'If': For a Lindenbaum closure relation $\vdash$ satisfying the conditions cited, we difine a class $\mathscr{V}$ determining $\vdash$ and closed under $\bar{\nabla}$ in two stages. First define $\mathscr{V}_{0}$ by: $v \in \mathscr{V}_{0}$ if $v=v_{W}$ for $W$ a maximal avoider relative to $\vdash$ or if $v=\bar{v}_{W}$ for $W$ a maximal avoider. Then let $\mathscr{V}$ be the least class of valuations (for the underlying set of $\vdash$ ) such that $\mathscr{V} \supseteq \mathscr{V}_{0}$ and $\mathscr{V}$ is closed under $\triangle$ and $\nabla$. By construction, then, $\mathscr{V}$ is closed under $\nabla$, and routine boolean-algebraic manipulations show that $\mathscr{V}$ is also closed under ${ }^{-}$, whence it follows that $\mathscr{V}$ is closed under $\bar{\nabla}$. It remains to show that $\mathscr{V}$ determines $\vdash$, i.e., that $X \vdash y$ iff for all $v \in \mathscr{V}$ with $v(X)=\mathrm{T}, v(Y)=\mathrm{T}$. Those elements $v_{W}$ (for $W$ a maximal avoider) in $\mathscr{V}_{0}$ show that if $X \not \forall y$, we have $v \in \mathscr{V}$ with $v(X)=\mathrm{T}$ and $v(Y)=\mathrm{F}$, since $\vdash$ is a Lindenbaum closure relation. Conversely, we must show that whenever $X \vdash y$ and $v(X)=\mathrm{T}$, then $v(Y)=\mathrm{T}$, if $v \in \mathscr{V}$; that is, that $\mathscr{V} \subseteq \mathscr{V}_{a l}(\vdash)$. This is clear for $v \in \mathscr{V}_{0}$ of the form $v_{W}$ ( $W$ a maximal avoider), and the proof of Thm. 2.1.3 showed that the assumption that $\vdash$ satisfies (Reverse ${ }^{\text {cc }}$ ) suffices for $\bar{v}_{\mathrm{w}}$ to belong to $\mathscr{V}_{a d}(\vdash)$ when $W$ is a maximal avoider. So $\mathscr{V}_{0} \subseteq \mathscr{V}_{a}(\vdash)$. For any closure relation, taking conjunctive combinations preserves consistency with that closure relation (Coro. 0.2.4 (i)), and disjunctive combinations of $\vdash$-consistent valuations are $\vdash$ -consistent since $\vdash$ is assumed to satisfy (Simp. Unions), by Th. 2.2.2.

'Only if': Since $\bar{v}=v \bar{\nabla} v$ and $v_{1} \nabla v_{2}=\left(v_{1} \bar{\nabla} v_{2}\right) \bar{\nabla}\left(v_{1} \bar{\nabla} v_{2}\right)$, a class of valuations closed under $\bar{\nabla}$ is closed under $\nabla$ and ${ }^{-}$, so that if $\vdash$ is determined by a $\bar{\nabla}$-closed class it satisfies (Reverse ${ }^{\mathrm{cc}}$ ) nad (Simp. Unions) by Thm. 2.1.3 and Coro, 2.2.3 respectively.

In view of the Galois-dual weak functional completeness of $\bar{\nabla}$, for each generalized rule-like condition $\mathbf{C}$ we have seen to be satisfied by any Lindenbaum closure relation determined by a class of valuations closed under the Galois-duals of some non-zero-place boolean connective, $\mathbb{C}$ follows from (Simp. Unions) and $\left(\right.$ Reverse $\left.^{c c}\right)$. By way of example, consider the condition (Flip-around ${ }^{c c}$ ), satisfied by all - we do not say 'all and only' - those closure relations determined by a -closed class of valuations. Given the antecedent of that condition: $X_{0}, Y_{1} \vdash y$, we infer by (Simp. Unions) that either $X_{0} \vdash y$ or $X_{1} \vdash y$. From the first of these disjuncts we obtain $X_{0} \vdash^{\text {cc }} y, X_{1}$, by $(\mathbb{M})$, which is one of the two disjuncts of the consequent of (Flip-around ${ }^{c c}$ ), while from the second disjunct by (Reverse ${ }^{\text {cc }}$ ) we obtain $y \vdash{ }^{\mathrm{cc}} X_{1}$, and so, by (M) again, $X_{0}, y \vdash^{\mathrm{cc}} X_{1}$, the remaining disjunct needed for (Flip-around ${ }^{\mathrm{cc}}$ ).

The restriction to non-zero-place operations is essential here. For the passage from weak to strong (Galois-dual) functional completeness we need in addition to 
the hypothesis that $\mathscr{V}$, determining $\vdash$, is closed under (say) $\bar{\nabla}$, also that $\mathscr{V} \neq \varnothing$ in order to infer that $v_{\mathrm{F}}$ and $v_{\mathrm{T}}$ belong to $\mathscr{V}$. The closure relation $\vdash_{1}$ on $U$ such that $X \vdash{ }_{1} y$ for all $X \subseteq U, y \in U$ obviously satisfies (Simp. Unions) and almost as obviously - see below - satisfies (Reverse ${ }^{\mathrm{cc}}$ ), but $\vdash_{1}$ is determined by no class of valuations containing $v_{\mathrm{F}}$ since $\vdash_{1}$ does not meet the 'atheorematicity' condition noted in Thm. 2.5.3 (i) to be necessary (and indeed sufficient) for determination by $\mathscr{V}$ with $v_{\mathrm{F}} \in \mathscr{V}$. (We choose this notation because $\vdash_{1}$ and the $\vdash_{0}$ of 0.3 are the unit and the zero of the lattice of all closure relations on a given $U$, with $\subseteq$ as the partial ordering; see [28], $\$ 1.5$ for more in this vein.) In fact, we could use this example again to illustrate the fallaciousness of arguing $a$ la (i)-(ii)- $\therefore$ (iii) above. Since every closure relation is determined by a class of valuations containing $v_{\mathrm{T}}$ this holds for $\vdash_{1}$. $\vdash_{1}$ satisfies, as remarked, the condition (Reverse ${ }^{\mathrm{cc}}$ ) and is therefore determined by a class of valuations closed under ${ }^{-}$. The fallacious conclusion would then be that $\vdash_{1}$ is determined by a class of valuations containing $v_{\mathrm{T}}$ and closed under the operation ${ }^{-}$, whose incorrectness we have already seen in observing that $\vdash_{1}$ is determined by no class containing $v_{\mathrm{F}}$ (since $\bar{v}_{\mathrm{T}}=v_{\mathrm{F}}$ ). As ever the explanation lies in the phenomenon of multiple determination. The facts are that $\vdash_{1}$ is determined by $\varnothing$ (the empty class of valuations), which is closed under - but does not contain $v_{\mathrm{T}}$, as well as by $\left\{v_{\mathrm{T}}\right\}$ which contains $v_{\mathrm{T}}$ but is not closed under $^{-}$(by Coro. 0.2.4 (iii)).

As intimated, it is not completely obvious that $\vdash_{1}$ must satisfy $\left(\right.$ Reverse ${ }^{c c}$ ). Since the antecedent, $X \vdash y$, of this condition always holds for $\vdash=\vdash{ }_{1}$, we must show that the consequent, $y \vdash^{\mathrm{cc}} X$ also always holds. If $x \in X$ then from the fact that $y \vdash{ }_{1} x$, (M) delivers the conclusion that $y \vdash{ }_{1}^{c c} X$. The less obvious case is that in which $X=\varnothing$. We encountered $\varnothing$ on the left of ' $\vdash{ }^{c c}$ ' in the discussion following Thm. 2.5.3, and we need now to consider its appearance on the right. The definition of $\vdash^{\mathrm{cc}}$ as applied to the case of $Y \vdash^{\mathrm{cc}} \varnothing$ gives us that for all $Y^{\prime} \supseteq Y$ and for all $z \in U:$

$$
\text { if } Y^{\prime}, u \vdash z \text { for each } u \in \varnothing \text {, then } Y^{\prime} \vdash z
$$

Since there are no $u \in \varnothing$, the condition here is vacuous, and what we have ended up saying is simply that for all $Y^{\prime} \supseteq Y$ and all $z$ : $Y^{\prime} \vdash z$, which is equivalent (by $(\mathbb{M})$ to the claim that for all $z$, we have $Y \vdash z$. This claim is clearly correct for $\vdash=\vdash{ }_{1}$, for all $Y$, and so certainly for the case of $Y=\{y\}$ needed to verify the consequent of (Reverse ${ }^{\mathrm{cc}}$ ).

Setting the case of $\vdash_{1}$ to one side, we have seen here an interesting consequence of $\left(\right.$ Reverse $\left.^{\mathrm{cc}}\right)$ for arbitrary closure relations which satisfy that condition, putting $X=\varnothing$ in the condition: if $\vdash y$ then $\vdash z$, for all $y, z$. Equivalently: $C_{\vdash}(\varnothing)$ $=\varnothing$ or $C_{\vdash}(\varnothing)=U$. The second alternative here holds just in case $\vdash \vdash_{1}$. So (Reverse $^{c c}$ ) is satisfied by a closure relation only if that relation is 'atheorematic' or else is the universal closure relation $\mathscr{P}(U) \times U$ on its underlying set $U$.

Our treatment of the case of $\bar{\nabla}$ has traded heavily on the weak functional 
completeness phenomenon and more specifically on the fact that a class of valuations is closed under $\bar{\nabla}$ iff it is closed under $\nabla$ and ${ }^{-}$. The same would have applied in the case of the poset-dual operation $\bar{\triangle}$, the Galois-dual of the connective $\wedge$ of alternative denial, which also goes by the names 'Sheffer stroke' and 'nand'. Thus we have $v_{1} \bar{\triangle} v_{2}(u)=\mathrm{T}$ iff $v_{1}(u)=\mathrm{F}$ or $v_{2}(u)=\mathrm{F}$. The easy parts of the discussion-summarized in Thm. 2.5.9-concern the first two of these three conditions:

(Nand)

$\left(\right.$ Nand $\left.^{\exists}\right)$

$$
X_{0}, X_{1} \vdash Y \text { implies } Y \vdash X_{0} \text { or } Y \vdash X_{1}
$$

$\left(\right.$ Nand $\left.^{\exists \mathrm{cc}}\right)$

$$
X_{0}, X_{1} \vdash^{\exists} Y \text { implies } Y \vdash^{\exists} X_{0} \text { or } Y \vdash^{\exists} X_{1}
$$$$
X_{0}, X_{1} \vdash^{\exists} Y \text { implies } Y \vdash^{c c} X_{0} \text { or } Y \vdash^{c c} X_{1}
$$

But we should also include another case (aside from $\sim$ and $\nabla$, to which we return shortly) of a (weakly) functionally incomplete operation; namely that Galois-dual to the connective 'and not'; let us notate this by ' $\Rightarrow$ '. That is, we define $v_{1} \bar{\Rightarrow} v_{2}(u)=\mathrm{T}$ iff $v_{1}(u)=\mathrm{T}$ and $v_{2}(u)=\mathrm{F}$. This naturally leads to the conditions:

(NImpl)

$$
X \vdash Y_{0}, Y_{1} \text { implies } X \vdash Y_{0} \text { or } Y_{1} \vdash X \text {. }
$$

$\left(N \operatorname{Impl} l^{\exists}\right)$

$$
X \vdash^{\exists} Y_{0}, Y_{1} \text { implies } X \vdash^{\exists} Y_{0} \text { or } Y_{1} \vdash^{\exists} X \text {. }
$$

$\left(N I m p l^{\exists c c}\right)$

$$
X \vdash^{\exists} Y_{0}, Y_{1} \text { implies } X \vdash^{c c} Y_{0} \text { or } Y_{1} \vdash^{c c} X \text {. }
$$

Then the techniques of our earlier proofs of analogous results give:

Theorem 2.5.9. (i) A generalized closure relation $\vdash$ satisfies (Nand) iff $\mathscr{V}_{\text {at }}(\vdash)$ is closed under $\bar{\triangle}$; a closue relatio $\vdash$ satisfies $\left(\right.$ Nand $\left.^{\exists}\right)$ iff $\mathscr{T}_{\text {at }}(\vdash)$ is closed under $\bar{\triangle}$.

(ii) A generalized closure relation $\vdash$ satisfies (NImpl) iff $\mathscr{T}_{\text {at }}(\vdash)$ is closed under $\bar{\Rightarrow}$; a closure relation $\vdash$ satisfies $\left(N \operatorname{Impl} l^{\exists}\right)$ iff $\mathscr{V a t}(\vdash)$ is closed under $\bar{\Rightarrow}$.

When it comes to the conditions delivered by the ' $\exists$ cc' strategy, (Nand ${ }^{\exists c c}$ ) and $\left(\mathrm{NImpl}{ }^{\exists \mathrm{cc}}\right.$ ) - as well as that left over from the start of the present subsection $\left({ }^{(E x c l}{ }^{\exists c c} X_{0}, X_{1} \vdash^{\exists} Y_{0}, Y_{1}\right.$ implies $X_{0}, Y_{1} \vdash^{c c} X_{1}, Y_{0}$ or $X_{1}, Y_{1} \vdash^{c c} X_{0}, Y_{0}$

we are not in so happy a position. Recall that the idea of the ' $\exists$ cc' strategy is to deliver from a conditon on generalized closure relations which is necessary and sufficient for those $\vdash$ satisfying it to have $\mathscr{T}_{a t}(\vdash)$ closed under a given operation, a condition on closure relations which is necessary and sufficient for the closure relations - or at least the Lindenbaum closure relations - satisfying it to be determined by some class of valuations closed under that same operation. We have seen this strategy work in the case of ${ }^{-}$(Thm. 2.1.3), $\nabla$ (Coro. 2.2.3), $\triangle$ (discussion following Thm. 2.3.1), $\Rightarrow$ (Thm. 2.4.4), when applied to (Reverse), (Left Unions), (Right Unions) and (Impl) respectively; but we saw it fail in the case of 
$\bar{\nabla}$ (Coro.2.5.7), when applied to (Nor). The author does not know whether the strategy succeeds as applied to (Nand) and (Excl) - that is, whether or not the conditions (Nand ${ }^{\exists c c}$ ) and $\left(\mathrm{Excl}^{\exists \mathrm{cc}}\right)$ are sufficient to guarantee at least all Lindenbaum closure relations satisfying them to be determined by some class of valuatons closed, respectively, under $\bar{\triangle}, \nabla$. In the case of (NImpl), we can show that the strategy fails, by means of an argument like that given in the proof of Coro. 2.5.7 above:

Proposition 2.5.10. Any closure relation satisfying (Reverse ${ }^{\mathrm{cc}}$ ) satisfies $\left(N \operatorname{Impl} l^{\exists c c}\right)$.

Proof. Suppose that $\vdash$ satisfies $\left(\right.$ Reverse $^{c c}$ ) and that we have $X \vdash^{\exists} Y_{0}, Y_{1}$. We must show that either $X \vdash^{c c} Y_{0}$ or $Y_{1} \vdash^{c c} X$. Since $X \vdash^{\exists} Y_{0}, Y_{1}$, either $X \vdash^{\exists} Y_{0}$ or else $X \vdash^{\exists} Y_{1}$. In the former case we can weaken the ' $\exists$ ' to a 'cc', while in the latter, $\left(\right.$ Reverse ${ }^{\mathrm{cc}}$ ) given $Y_{1} \vdash^{\mathrm{cc}} X$.

Corollary 2.5.11. Satisfaction of (NImpl ${ }^{\exists \mathrm{cc}}$ ) by a closure relation is not sufficient for the existence of a determining class of valuations closed under $\bar{\rightrightarrows}$.

Proof. Exactly as in the proof of Coro. 2.5.7, we observe that the closure relation $\vdash$ described in Example 2.1.4 satisfies (Reverse ${ }^{\mathrm{cc}}$ ) ; so by Prop. 2.5.10 $\vdash$ satisfies $\left(\mathrm{NImpl}^{\exists \mathrm{cc}}\right)$. We saw in the proof of 2.5.7 that $\vdash$ is not determined by any class of valuations closed under $\nabla$. It follows that $\vdash$ is not determined by any class of valuations closed under $\bar{\Rightarrow}$, since $v_{1} \nabla v_{2}=v_{1} \bar{\rightrightarrows}\left(v_{1} \bar{\Rightarrow} v_{2}\right)$.

To resolve the analogous question for (NAnd $\left.{ }^{\exists c c}\right),\left(\operatorname{Excl}^{\exists c c}\right)$, and for that matter, (Flip-around ${ }^{\exists c c}$ ), what would be desirable would be a general result detailing the conditions under which the ' $\exists$ cc' strategy is successful. It was with the absence of such a result that we described (in the words introducing $\S 2$ ) this section as providing not so much a general theory as a survey of some of the data to be subsumed by such a theory. We have, however, done enough to be able to return to Problem 1.1.2 in $\S 3$ and offer a solution. That solution appears in Thm. 3.1 .2 , and the same idea works in cases, such as that of $\bar{\Rightarrow}$, in which the ' $\exists \mathrm{cc}$ ' strategy fails. The analogue of the conditions collectively called ' (Match)' in 3.1 for the case of $\bar{\Rightarrow}$, are conditions of the form

$$
\Gamma \vdash{ }^{\exists} \bar{\Gamma} \text { implies } W_{1} \vdash{ }^{c c} \bar{W}_{1} \text { or } \ldots \text { or } W_{n} \vdash{ }^{c c} \bar{W}_{n}
$$

In which $\Gamma$ is replaced by a polynomial built up from variables $W_{1}, \ldots, W_{n}$ by

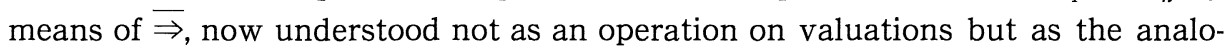
gous operation on subsets of $U: X \bar{\Rightarrow} Y=X \cap \bar{Y}$. One such condition, for example, would be the following (taking $n=4$ ): 


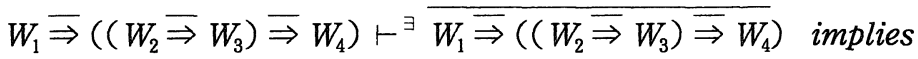

$$
\begin{aligned}
& W_{1} \vdash^{\mathrm{cc}} \bar{W}_{1} \text { or } W_{2} \vdash^{\mathrm{cc}} \bar{W}_{2} \text { or } W_{3} \vdash^{\mathrm{cc}} \bar{W}_{3} \text { or } W_{4} \vdash^{\mathrm{cc}} \bar{W}_{4}
\end{aligned}
$$

A routine adaptation of the 'if' part of the proof of Thm. 3.1.2 shows that each such condition is satisfied by any closure relation $\vdash$ for which there is some determining class of valuations closed under $\bar{\Rightarrow}$, and the 'only if' part of that proof yields a proof that any Lindenbaum closure relation satisfying all of the conditions described is determined by some class $\rightrightarrows$-closed class of valuations. The same technique works for the Galois-dual of any possible boolean connective (including for example the operation $\underline{\nabla}$ ), though the quasi-syntactic conditions obtained are admittedly much less elegant that the single generalized rule-like conditions we have obtained for cases in which the ' $\exists$ cc' strategy is known to succeed. (In fact, the conditions (Match) for the case of $\sim$ are slightly less cumbersome than those

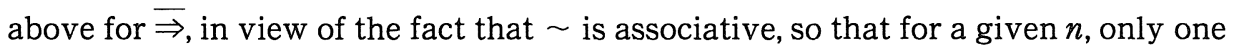
condition is required. In 3.2 , we will consider further a simplification, in the shape of a condition-schema we call '(Multi-flip)'.)

\subsection{The Provenance of the Conditions on Generalized Closure Relations}

In discussing the success of the ' $\exists \mathrm{cc}$ ' strategy for obtaining conditions on closure relations, we have presumed available a condition on generalized closure relations necessary and sufficient for their classes of consistent valuations to be closed under whatever operation is at issue. This raises the question of where we get those conditions from in the first place. Here we describe a general procedure which always delivers an appropriate such condition, though the condition delivered is typically more complicated than (though equivalent to) the conditions we have been citing to this end in 2.1-2.5. We begin by repeating a conditions for which this matter of greater complexity does not arise, namely, the condition (Flip-around) from 1.2:

(Flip-around) $X_{0}, X_{1} \vdash Y_{0}, Y_{1}$ implies $X_{0}, Y_{0} \vdash X_{1}, Y_{1}$ or $X_{0}, Y_{1} \vdash X_{1}, Y_{0}$

In the interests of describing our uniform general procedure, we will subject the above formulation to some re-lettering, in particular so that its consequent looks like this:

$$
Z_{\mathrm{LL}}, Z_{\mathrm{LR}} \vdash Z_{\mathrm{RL}}, Z_{\mathrm{RR}} \text { or } Z_{\mathrm{LL}}, Z_{\mathrm{RL}} \vdash Z_{\mathrm{LR}}, Z_{\mathrm{RR}}
$$

The subscripting is explained as follows: the (set-) variable ' $Z_{\mathrm{LL}}$ ' appears on the (' $\mathrm{L}$ ' for) left of the $\vdash$ in the first as well as the second disjunct, while ' $Z_{\mathrm{LR}}$ ' appears on the left in the first disjunct but on the right in the second. Similarly, an ' $R$ ' in the first subscripted position indicates an appearance on the right in the first disjunct. (Note that this talk of left and right has nothing to do with the left and right of the Galois connexions introduced in 0.2 ; left/right interchanges here pertain to 
poset-duality, not Galois-duality.)

All of the conditions our general procedure delivers for the closure of $\mathscr{V}_{a l}(\vdash)$ under a binary operation have the same disjunctive consequent as is displayed above, differing only in their antecedents. In particular, for (Fliparound) as formulated above, ' $X_{0}^{\prime}$ ' appears on the left in both disjuncts, so it is now written as ' $Z_{\mathrm{LL}}$ ', ' $Y_{1}$ ' appears first on the right, and then on the left, so it becomes ' $Z_{\mathrm{RL}}$ ', and so on. Relettering (Flip-around) 's antecedent in the new variables, then, we have:

$$
Z_{\mathrm{LL}}, Z_{\mathrm{RR}} \vdash Z_{\mathrm{LR}}, Z_{\mathrm{RL}} \text { implies } Z_{\mathrm{LL}}, Z_{\mathrm{LR}} \vdash Z_{\mathrm{RL}}, Z_{\mathrm{RR}} \text { or } Z_{\mathrm{LL}}, Z_{\mathrm{RL}} \vdash Z_{\mathrm{LR}}, Z_{\mathrm{RR}}
$$

The fact that if $\mathscr{V}_{a}(\vdash)$ is closed under $\sim$ then (Flip-around) must be satisfied as shown in the 'if' part of the proof of Thm. 1.2.1 - can then be seen as the application of a general line of reasoning, which we introduce by replicating that part of the proof. Suppose that $Z_{\mathrm{LL}}, Z_{\mathrm{LR}} \not \forall Z_{\mathrm{RL}}, Z_{\mathrm{RR}}$ and $Z_{\mathrm{LL}}, Z_{\mathrm{RL}} \not \nvdash Z_{\mathrm{LR}}, Z_{\mathrm{RR}}$. Then we have valuations $v_{1}$ and $v_{2}$ in $\mathscr{V}_{a}(\vdash)$ with $v_{1}\left(Z_{\mathrm{LL}} \cup Z_{\mathrm{LR}}\right)=\mathrm{T}, v_{1}\left(Z_{\mathrm{RL}} \cup Z_{\mathrm{RR}}\right)=\mathrm{F}$, $v_{2}\left(Z_{\mathrm{LL}}, Z_{\mathrm{RL}}\right)=\mathrm{T}, v_{2}\left(Z_{\mathrm{LR}}, Z_{\mathrm{RR}}\right)=\mathrm{F}$. So, the elements of $Z_{\alpha \beta}$ are true on $v_{1}$ whenever $\alpha=L$, false on $v_{1}$ when $\alpha=R$, true on $v_{2}$ whenever $\beta=L$, and false on $v_{2}$ when $\beta=R$. Since the conditions we are considering will all of them have the same disjunctive cnsequent, this will be the situation in all cases, and it will be a great aid to succinctness if we adopt the following convetion: a lower case Greek letter appearing in subscript position on ' $Z$ ' and also as the name of a truth-value is to interpreted either as standing for ' $\mathrm{L}$ ' in its subscript appearances and ' $\mathrm{T}$ ' when not subscripted, or else as standing for ' $R$ ' in its subscripted appearances and for ' $F$ ' when not subscripted. With this convention in force, we have $v_{1}\left(Z_{\alpha \beta}\right)=\alpha$ and $v_{2}\left(Z_{\alpha \beta}\right)=\beta$. (We only use the notation $v_{i}\left(Z_{\alpha \beta}\right)$ when $v_{i}$ assigns the same value to all elements of $Z_{\alpha \beta}$.)

The way the antecedent of the relettered version of (Flip-around) above is dictated by the truth-function $f_{\leftrightarrow}$ associated over $\leftrightarrow$-boolean valuations with the connective $\leftrightarrow$ whose Galois-dual is at issue here, represents an application of the following general procedure for obtaining what we call the canonical antecedent of a condition designed to capture syntactically the closure of $\mathscr{V}_{a t}(\vdash)$ under the operation \# Galois dual to a connective \# with associated truth-function $f_{\# \text {. (Here }}$ we have used the notation of 0.3.) The consequent of this canonically obtained condition is of course (18). We have to say for each of the four Z-terms in the consequent, on which side of the ' $\vdash$ ' in the antecedent it is to go:

$$
\begin{aligned}
& \text { If } f_{\#}(\alpha, \beta)=\mathrm{T} \text { then put } Z_{\alpha \beta} \text { on the left; } \\
& \text { if } f_{\#}(\alpha, \beta)=\mathrm{T} \text { then put } Z_{\alpha \beta} \text { on the right. }
\end{aligned}
$$

Now we have $v_{1} \Uparrow v_{2}\left(Z_{\alpha \beta}\right)=f_{\#}\left(v_{1}\left(Z_{\alpha \beta}\right), v_{2}\left(Z_{\alpha \beta}\right)\right)$

$$
=f_{\#}(\alpha, \beta)
$$

But if $f_{\#}(\alpha, \beta)=\mathrm{T}$, then by (19), $Z_{\alpha \beta}$ went on the left, and if $f_{\#}(\alpha, \beta)=\mathrm{F}$, then 
$Z_{\alpha \beta}$ went on the right, in the canonical antecedent. So $v_{1} \widehat{\#} v_{2}$ verifies everything on the right but nothing on the left of the $\vdash$ in the canonical antecedent. The antecedent is therefore refuted by the existence of any $v_{1}, v_{2}$, respectively refuting the two disjuncts of the consequent $(=(18))$. The whole conditional obtained by this procedure is thus bound to be satisfied by any generalized closure relation whose class of consistent valuations is closed under $\widehat{\#}$.

We now need to show that our uniform conditions on generalized closure relations, derived by taking the canonical antecedent given by (19) and the fixed consequent (18), are also sufficient to guarantee that $\vdash$ satisfying them have

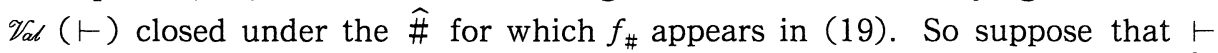
satisfies the appropriate canonical condition but $\mathscr{V}_{a t}(\vdash)$ is not closed under \# Thus we have $v_{1}, v_{2} \in \mathscr{V}_{a l}(\vdash)$ with $v_{1} \# v_{2} \notin \mathscr{V}_{a l}(\vdash)$, so there are $X, Y$ such that

(i) $X \vdash Y$ but (ii) $v_{1} \# v_{2}(X)=\mathrm{T}$ and (iii) $v_{1} \widehat{\#} v_{2}(Y)=\mathrm{F}$

Put $Z_{\mathrm{LL}}=T\left(v_{1}\right) \cap T\left(v_{2}\right), Z_{\mathrm{LR}}=T\left(v_{1}\right) \cap \overline{T\left(v_{2}\right)}, Z_{\mathrm{RL}}=\overline{T\left(v_{1}\right)} \cap T\left(v_{2}\right), Z_{\mathrm{LL}}=\overline{T\left(v_{1}\right)} \cap$ $\overline{T\left(v_{2}\right)}$. This means, as before, that $v_{1}\left(Z_{\alpha \beta}\right)=\alpha, v_{2}\left(Z_{\alpha \beta}\right)=\beta$, and that $v_{1}$ and $v_{2}$ respectively refute the first and second disjuncts of (18), since $v_{1}, v_{2} \in \mathscr{V}_{a t}(\vdash)$. As we are supposing $\vdash$ to satisfy the canonical condition for $\widehat{\#}$, the antecedent given by (19) in terms of $f_{\#}$ must also fail. For our contradiction, we show that $X$ is included in the right-hand side of that antecedent and $Y$ in the left-hand side. (Thus we get the contradiction because of (M).) Take $x \in X$. By (ii), $v_{1}$ $v_{2}(x)=\mathrm{T}$. But $v_{1} \Uparrow v_{2}(x)=f_{\#}\left(v_{1}(x), v_{2}(x)\right)$. Putting $\alpha$ for $v_{1}(x)$ and $\beta$ for $v_{2}(x)$, then, we have $f_{\#}(\alpha, \beta)=\mathrm{T}$, so (19) dictated that $Z_{\alpha \beta}$ ended up on the left of $\vdash$ in the canonical antecedent for \#. Further, $x \in Z_{\alpha \beta}$, since $Z_{\alpha \beta}$ comprises all elements $u$ for which $v_{1}(u)=\alpha$ and $v_{2}(u)=\beta$. So every element of $X$ appears in the right-hand side of the antecedent. The argument that every element of $Y$ appears on the left-hand side is similar, and we omit it here.

Although we have described the procedure for obtaining our canonical conditions on generalized closure relations apt for binary operations $\widehat{\#}$, extending the treatment to $n$-ary operations is routine. When $n=3$ (so that we have some ternary connective \# with and a notion of \#-boolean valuation associating \# with a truth-function $f_{\#}$ ), for example, the fixed consequent (18) becomes a threefold disjunction whose first disjunct reads:

$$
Z_{\mathrm{LLL}}, Z_{\mathrm{LLR}}, Z_{\mathrm{LRL}}, Z_{\mathrm{LRR}} \vdash Z_{\mathrm{RRR}}, Z_{\mathrm{RRL}}, Z_{\mathrm{RLR}}, Z_{\mathrm{RLL}}
$$

with second (third) disjunct having on the left each of the $8 \mathrm{Z}$-term with ' $\mathrm{L}$ ' in the second (third) position, and on the right those with ' $R$ ' in the second (third) position. The antecedent is then obtained by the obvious adaptation of (19), as

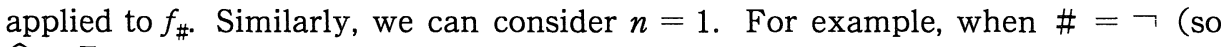
$\widehat{\#}$ is $^{-}$) from (19) reformulated for the one-place case, $f_{\neg}$ we get the condition

$$
Z_{\mathrm{R}} \vdash Z_{\mathrm{L}} \text { implies } Z_{\mathrm{L}} \vdash Z_{\mathrm{R}}
$$


which is a relettering of the condition (Reverse) we employed to treat this case in 2.1 .

In fact the only conditions on generalized closure relations that we have employed which are (to within relettering) those delivered by the uniform procedure described above are (Flip-around), (Excl) and (Reverse): conditions for the Galois-duals of boolean connectives \# where $f_{\#}$ is one of Post's 'alternating' truth functions [21]. (Though this does not seem coincidental, the author does not have an explanation for it.) In general, the canonical conditions are more complicated than those we have chosen to employ instead. The situation in this regard is similar to that when we use analogues of (19) to provide a general recipe for obtaining conditions on $\vdash$ for the connectives themselves (as with Segerberg's account in terms of type-determination in [24], [25]): such recipes deliver, when we want determination by the class of $\vee$-boolean valuations, for example, the conditions in (22), which have then to be further simplified to get the more familiar and more natural set in (23)

$$
\begin{gathered}
x, y \vdash x \vee y ; x \vdash x \vee y, y ; y \vdash x \vee y, x ; x \vee y \vdash x, y \\
x \vdash x \vee y ; y \vdash x \vee y ; x \vee y \vdash x, y
\end{gathered}
$$

Segerberg remarks in [25], p.562, on the comparative clumsiness of some natural deduction rules derived by a general method suited for a proof that the method always given a sound and complete system of rules, when these are compared with the (equivalent) sets of rules for familiar connectives that first come to mind. We are now dealing with the Galois-dual phenomenon, and might as well illustrate in with the operation Galois dual to $\vee$, namely $\nabla$.

Our condition in 5.2, which might well deserve to be called the first that comes to mind' for the case of disjunctive combinations, was the following:

(Left Unions) $X_{0}, X_{1} \vdash Y$ implies $X_{0} \vdash Y$ or $X_{1} \vdash Y$

Compare it with the canonical condition we get by taking our fixed consequent (18) and the canonical antecedent dictated by (19) for the case of $\nabla\left(\right.$ via $f_{\mathrm{V}}$ ):

(24) $Z_{\mathrm{LL}}, Z_{\mathrm{LR}}, Z_{\mathrm{RL}} \vdash Z_{\mathrm{RR}}$ implies $Z_{\mathrm{LL}}, Z_{\mathrm{LR}} \vdash Z_{\mathrm{RL}}, Z_{\mathrm{RR}}$ or $Z_{\mathrm{LL}}, Z_{\mathrm{RL}} \vdash Z_{\mathrm{LR}}, Z_{\mathrm{RR}}$

Not only do we have four variables over subsets of $U$ instead of three, but these variables make far more appearances than do the variables in (Left Unions): it both respects, an exact analogy with (22) vs. (23).

The general argument above for the necessity and sufficiency of the canonical conditions, coupled for the present case with Thm. 2.2.1, show that (Left Unions) and (24) are equivalent conditions on generalized closure relations. Showing this equivalence without recourse to semantic notions is not simple. There is a straightforward 'generalized deduction' of (24) from (Left Unions): suppose $Z_{\mathrm{LL}}, Z_{\mathrm{LR}}, Z_{\mathrm{RL}} \vdash Z_{\mathrm{RR}}$. Rewrite this as

$$
Z_{\mathrm{LL}} \cup Z_{\mathrm{LR}}, Z_{\mathrm{LL}} \cup Z_{\mathrm{RL}} \vdash Z_{\mathrm{RR}}
$$


to which we can apply (Left Unions), obtaining

$$
Z_{\mathrm{LL}} \cup Z_{\mathrm{LR}} \vdash Z_{\mathrm{RR}} \text { or } Z_{\mathrm{LL}} \cup Z_{\mathrm{RL}} \vdash Z_{\mathrm{RR}}
$$

from which the consequent of (24) follows by ( $M$ ).

In the reverse direction, the only proof known to the author would in effect use thinly disguised semantic considerations in reasoning (toward a contradiction) from the assumption that $\vdash$ satisfies (24) but we have $X_{0}, X_{1} \vdash Y$ while $X_{0} \vdash Y$ and $X_{1} \not \nvdash Y$, to the existence of $X_{0}^{+} \supseteq X_{0}$ with $\bar{X}_{0}^{+} \supseteq Y$ and $X_{1}^{+} \supseteq X_{1}$ with $\bar{X}_{1}^{+} \supseteq Y$ such that

$$
X_{0}^{+} \not-\bar{X}_{0}^{+} \text {and } X_{1}^{+} \not \nvdash \bar{X}_{1}^{+}
$$

by $\left(\mathbb{T}^{+}\right)$. The fact that $X_{i}^{+} \cup \bar{X}_{0}^{+}=U$ means we are essentially dealing with $T(v)$ and its complement, for $v \in \mathscr{V}_{\text {at }}(\vdash)$, whence the above remark about semantic considerations. To continue the argument anyway: since $X_{0}, X_{1} \vdash Y$, we have, by (M), $X_{0}^{+}, X_{1}^{+} \vdash Y$, which we rewrite as

$$
X_{0}^{+} \cap X_{1}^{+}, X_{0}^{+} \cap \bar{X}_{1}^{+}, \bar{X}_{0}^{+} \cap X_{1}^{+} \vdash Y .
$$

Now we are in a position to invoke our canonical condition (24), and infer (29) $X_{0}^{+} \cap X_{1}^{+}, X_{0}^{+} \cap \bar{X}_{1}^{+} \vdash \bar{X}_{0}^{+} \cap X_{1}^{+}, Y$ or $X_{0}^{+} \cap X_{1}^{+}, \bar{X}_{0}^{+} \cap X_{1}^{+} \vdash X_{0}^{+} \cap X_{1}^{+}, Y$.

By $(\mathbb{M})$, this time on the right, we have

$$
X_{0}^{+} \cap X_{1}^{+}, X_{0}^{+} \cap \bar{X}_{1}^{+} \vdash \bar{X}_{0}^{+}, Y \text { or } X_{0}^{+} \cap X_{1}^{+}, \bar{X}_{0}^{+} \cap X_{1}^{+} \vdash \bar{X}_{1}^{+}, Y \text {. }
$$

and simplifying the left-hand sides:

$$
X_{0}^{+} \vdash \bar{X}_{0}^{+}, Y \text { or } X_{1}^{+} \vdash \bar{X}_{1}^{+}, Y \text {. }
$$

Since $Y \subseteq \bar{X}_{0}^{+}$and $Y \subseteq \bar{X}_{1}^{+}$, we can drop the ' $Y$ ' from both left-hand sides, giving the desired contradiction with (27).

\section{$\S$ 3. Determination by Classes of Valuations Closed under Matching}

\subsection{An Approach Suggested by the Preceding Discussion}

After the excursion of $\S 2$, surveying some other cases of determination of closure relations by classes of valuations closed under operations Galois-dual to the familiar boolean connectives, we resume our discussion of the specific case of from the point at which we left it in 1.2. The most useful of the examples we have worked through is that of closure under ${ }^{-}$, for which case we found (Thm. 2.1.3) the following condition necessary and sufficient for a Lindenbaum closure relation to be determined by a class of valuations closed under ${ }^{-}$:

$\left(\right.$ Reverse $\left.^{\mathrm{cc}}\right)$

$$
X \vdash y \text { implies } y \vdash^{c c} X
$$


The strategy of the hard part of the proof was to take as the determining class the set of characteristic functions of maximal avoiders and of their complements. This required making sure that the valuations obtained in the latter way were indeed consistent with a $\vdash$ satisfying $\left(\right.$ Reverse $^{\text {cc }}$ ), which is equivalent to saying that if $W$ is a maximal avoider, then $\bar{W}$ is $\vdash$-closed. In view of Prop. 0.5.1, we can express this requirement directly as a rule-like condition on $\vdash$ :

(Complements) $\bar{W} \vdash^{\exists} W$ implies $W\left\ulcorner^{\mathrm{cc}} \bar{W}\right.$

Contraposing: if $W \nvdash^{c c} \bar{W}$, then $\bar{W} \nvdash^{\exists} W$; which means that if $W$ is a maximal avoider then $\bar{W}$ is $\vdash$-closed (by Prop. 0.5.1 since $W$ is the complement of the complement of $W$ ).

Proposition 3.1.1. A Lindenbaum closure relation satisfies (Complements) iff it satisfies (Reverse ${ }^{\mathrm{cc}}$ ).

Proof. The 'if' part holds for arbitrary closure relations $\vdash$. Suppose $\vdash$ satisfies (Reverse ${ }^{c c}$ ) and that we have the antecedent of (Complements), $\bar{W} \vdash^{\exists} W$, i.e., $\bar{W} \vdash y$ for some $y \in W$. By (Reverse ${ }^{\text {cc }}$ ), then, $y \vdash^{\text {cc }} \bar{W}$, whence by $(\mathbb{M})$, since $y \in W$, we obtain the consequent of (Complements): $W \vdash^{\text {cc }} \bar{W}$.

'Only if': Suppose $\vdash$ is a Lindenbaum closure relation satisfying (Complements), and that $X \vdash y$. We must show that $y \vdash{ }^{\mathrm{cc}} X$. Suppose otherwise; then for some $Y_{0} \supseteq\{y\}$ there is a $z$ such that $Y_{0}, x \vdash z$ for each $x \in X$, while $Y_{0} \nvdash z$. Since $\vdash$ is a Lindenbaum closure relation there is a $Y_{1} \supseteq Y_{0}$ which maximally avoids $z$. For this $Y_{1}$ we have $y \in Y_{1}$ and $X \subseteq \bar{Y}_{1}$; thus by (M) from the fact that $X \vdash y$ we have $\bar{Y}_{1} \vdash^{\exists} Y_{1}$, which by (Complements) implies $Y_{1} \vdash^{\mathrm{cc}} \bar{Y}_{1}$, contradicting the fact that $Y_{1}$ is a maximal avoider (with appeal to Prop. 0.5.1 (ii)).

To deal with determination by classes of valuations closed under $\sim$, we may proceed analogously, with a condition we shall call (Match) playing the role of (Complements) above. That is, we need to take characteristic functions of maximal avoiders and the results of applying the operation $\sim$ to them, as the valuations in our determining class. Arbitrary consistent valuations will not themselves do the job, since applying $\sim$ to a pair of such valuations need not yield a consistent valuation, as was observed in Prop. 1.2.14; nor would sticking with just maximal avoiders do, as 1.2.14 also showed. Hence the need for a 'mixed' approach, involving the two levels, as with (Complements). The analogous condition in the present case is:

(Match) $W_{1} \sim \ldots \sim W_{n} \vdash^{\exists} \overline{W_{1} \sim \ldots \sim W_{n}}$ implies $W_{1} \vdash^{\mathrm{cc}} \bar{W}_{1}$ or $\ldots$ or $W_{n} \vdash^{\mathrm{cc}} \bar{W}_{n}$

As usual, overlining is for complementation (relative to $U$ ) ; internal bracketing has been ommitted in ' $W_{1} \sim \ldots \sim W_{n}$ ', since $\sim$ is associative, $\sim$ here being the binary operation on sets which takes $X, Y$ to $X \sim Y=(X \cap Y) \cup(\bar{X} \cap \bar{Y})$. (Thus there is a double usage of ' $\sim$ ', as the match operation on pairs of valuations and as 
the 'complement of the symmetric difference' operation on pairs of sets. The use of ' $\sim$ ' in expressions like ' $\mathscr{V} \sim \mathscr{V}$ ' in 1.1 is of course quite different from the present use of this symbol in standing between names for subsets of $U$.) Note that $W_{1} \sim \ldots \sim W_{n}$ consists of those elements (of whatever underlying set $U$ is pertinent to the discussion) which fail to belong to an even number (possibly 0 ) of the sets $W_{1}, \ldots, W_{n}$. More accurately, since we do not exclude the possibility that $W_{i}=W_{j}$ for $i \neq j: u \in W_{1} \sim \ldots \sim W_{n}$ iff the cardinality the set of $i(1 \leq i \leq n)$ such that $u \in W_{i}$ is even. (Sometimes for brevity in what follows we use the looser formulation, but with this interpretation intended.)

For a given $n$, we refer to the condition that (Match) above holds for all $W_{1}, \ldots, W_{n}$ as (Match) ; to say that $\vdash$ satisfies (Match) is then to say that it satisfies the condition (Match) $)_{n}$ for every $n \geq 2$ (The condition (Match) 1 is automatically satisfied by any closure relation, since maxinal avoiders are always $\vdash$-closed.) The following proof employs the ' $T(v)$ ' notation introduced in 0.2 .

Theorem 3.1.2. A Lindenbaum closure relation $\vdash$ satisfies (Match) iff $\vdash$ is determined by some class of valuations closed under $\sim$.

Proof. 'If: Suppose $\mathscr{V}$ determines $\vdash$ and $\mathscr{V}$ is closed under $\sim$, and that we have $W_{i} \not \nvdash^{\mathrm{cc}} \bar{W}_{\imath}$ for $i=1, \ldots, n$. Then for each such $i$ there is a valuation $v_{i} \in \mathscr{V}$ with $v_{i}\left(W_{\imath}\right)=\mathrm{T}, v_{i}\left(\bar{W}_{i}\right)=\mathrm{F}$. That is, $T\left(v_{\imath}\right)=W_{\imath}$. Since $\mathscr{V} \subseteq \mathscr{V} a(\vdash)$, each set $T(v)$ is $\vdash$-closed, for $v \in \mathscr{V}$. But $T\left(v_{1} \sim \ldots \sim v_{n}\right)=T\left(v_{1}\right) \sim \ldots \sim T\left(v_{n}\right)=$ $W_{1} \sim \ldots \sim W_{n}$, and $\mathscr{V}$ is closed under $\sim$, so $v_{1} \sim \ldots \sim v_{n} \in \mathscr{V}$ and therefore $W_{1} \sim \ldots \sim W_{n}$ is $\vdash$-closed. So (by Prop. 0.5.1 (i)) $W_{1} \sim \ldots \sim W_{n} \vdash^{\exists} \overline{W_{1} \sim \ldots \sim W_{n}}$. 'Only if': Suppose that $\vdash$ is a Lindenbaum closure relation satisfying (Match). We obtain $\mathscr{V}$ determining $\vdash$ by collecting up all characteristic functions of maximal avoiders and of finite $\sim$-combinations $W_{1} \sim \ldots \sim W_{n}$ of such maximal avoiders $W_{1}, \ldots, W_{n}$. Clearly $\mathscr{V}$ is closed under $\sim$, and it remains only to check that $\mathscr{V}$ determines $\vdash$. This requires us to verify that the valuations $v_{1} \sim \ldots \sim v_{n}$ are consistent with $\vdash$, or equivalently, that the sets $W_{1} \sim \ldots \sim W_{n}$ are $\vdash$-closed. But this is guaranteed by the condition (Match), since for each $i(1 \leq i \leq n) W_{i}$ $\forall^{\mathrm{cc}} \bar{W}_{i}$ ( $W_{i}$ being a maximal avoider), and thus, appealing to that condition, $W_{1} \sim \ldots \sim W_{n} \nvdash^{\exists} \overline{W_{1} \sim \ldots \sim W_{n}}$.

Thus Thm. 3.1.2 represents our solution to Problem 1.1.2, restricted in scope to the class of Lindenbaum closure relations. In 3.2 we will give an alternative solution involving what is in some respects a more attractive condition than (Match).

We already had, in 1.2, some conditions on closure relations as candidates for playing the role played in Thm. 3.1.2 by (Match), namely, the conditions (equivalent by Thm. 1.2.7): 
$\left(\right.$ Flip-around $^{\mathrm{cc}}$ ) $X_{0}, X_{1} \vdash y$ implies $X_{0}, y \vdash{ }^{\mathrm{cc}} X_{1}$ or $X_{0} \vdash{ }^{\mathrm{cc}} X_{1}, y$

(Flip-around ${ }^{\exists c c}$ ) $X_{0}, X_{1} \vdash^{\exists} Y_{0}, Y_{1}$ implies $X_{0}, Y_{0} \vdash^{c c} X_{1}, Y_{1}$ or $X_{0}, Y_{1} \vdash^{c c} X_{1}, Y_{0}$

and the question arises as to how these conditions are related to (Match). They turn out to be equivalent - for Lindenbaum closure relations - to the $n=2$ case of (Match):

Proposition 3.1.3. A Lindenbaum closure relation $\vdash$ satisfies $\left(\right.$ Match $\left._{2}\right)$ iff $\vdash$ satisfies (Flip-around ${ }^{\mathrm{cc}}$ ).

Proof. 'If': We show that any closure relation satisfying (Flip-around) ${ }^{\mathrm{cc}}$ must satisfy (Match) $)_{2}$. Suppose that $\vdash$ satisfies (Flip-around) ${ }^{\mathrm{cc}}$ and that $W_{1} \sim W_{2} \vdash^{\exists}$ $\bar{W}_{1} \sim W_{2}$, with a view to showing that either $W_{1} \vdash{ }^{\mathrm{cc}} \bar{W}_{1}$ or $W_{2} \vdash^{\mathrm{cc}} \bar{W}_{2}$. The supposition means that for some $y \notin W_{1} \sim W_{2}$, we have: $W_{1} \cap W_{2}, \bar{W}_{1} \cap \bar{W}_{2} \vdash{ }^{\mathrm{cc}} y$. Then by $\left(\right.$ Flip-around ${ }^{\mathrm{cc}}$ ), we have either

$$
W_{1} \cap W_{2}, y \vdash^{c c} \bar{W}_{1} \cap \bar{W}_{2} \text { or (2) } W_{1} \cap W_{2} \vdash^{c c} y, \bar{W}_{1} \cap \bar{W}_{2}
$$

Now, since $y \notin W_{1} \sim W_{2}$, either $y \in W_{1} \cap \bar{W}_{2}$ or $y \in \bar{W}_{1} \cap W_{2}$. Suppose that $y \in W_{1} \cap \bar{W}_{2}$. From (1) by $(\mathbb{M})$ we have $W_{1}, y \vdash{ }^{\text {cc }} \bar{W}_{1}$, and thus, since on the present supposition $y \in W_{1}: W_{1} \vdash{ }^{\mathrm{cc}} \bar{W}_{1}$; from (2) by (M) we have $W_{2} \vdash^{\mathrm{cc}} y, \bar{W}_{2}$, and hence, since $y \in \bar{W}_{2}: W_{2} \vdash^{c c} \bar{W}_{2}$. Thus whichever of (1), (2), holds we have the result that $W_{1} \vdash{ }^{c c} \bar{W}_{1}$ or $W_{2} \vdash{ }^{c c} \bar{W}_{2}$. Soppose instead that $y \in \bar{W}_{1} \cap W_{2}$. In this case we reason to this same conclusion but via the intermediate steps $W_{2}, y \vdash{ }^{\text {cc }} \bar{W}_{2}$ (from (1)) and $W_{1} \vdash^{\text {cc }} y, \bar{W}_{1}$ (from (2)).

'Only if': Suppose that $\vdash$ is a Lindenbaum closure relation satisfying (Match) ${ }_{2}$, and that $X_{0}, X_{1} \vdash y$ while $X_{0}, y \not \nvdash^{c c} X_{1}$ and $X_{0} \not \nvdash^{c c} y, X_{1}$. Showing this to be impossible will show that Lindenbaum closure relations satisfying (Match) ${ }_{2}$ must satisfy (Flip-around $^{\mathrm{cc}}$ ). Let $X_{0}^{\prime}$ and $X_{0}^{\prime \prime}$ be the supersets of $X_{0} \cup\{y\}$ and of $X_{0}$, respectively, promised by the claims that $X_{0}, y \not \nvdash^{c c} X_{1}$ and $X_{0} \not \nvdash^{c c} y, X_{1}$, and $z^{\prime}$ and $z^{\prime \prime}$ be elements of $U$ for which

(1) $X_{0}^{\prime}, u \vdash z^{\prime}$ for each $u \in X_{1}$, while $X_{0}^{\prime} \nvdash z^{\prime}$; (2) $X_{0}^{\prime \prime}, u \vdash z^{\prime \prime}$ for each $u \in$ $X_{1} \cup\{y\}$, while $X_{0}^{\prime \prime} \nvdash z^{\prime \prime}$. Let $W^{\prime}$ and $W^{\prime \prime}$ be supersets of $X_{0}^{\prime}$ and $X_{0}^{\prime \prime}$ respectively which maximally avoid the elements (resp.) $z^{\prime}$ and $z^{\prime \prime}$. (Here we exploit the assumption that $\vdash$ is a Lindenbaum closure relation.) By Prop. 0.5.1, $W^{\prime} \nvdash^{\text {cc }} \bar{W}^{\prime}$ and $W^{\prime \prime} \nvdash^{c c} \bar{W}^{\prime \prime}$, and so, since $\vdash$ satisfies (Match) $)_{2}$, we have $W^{\prime} \sim W^{\prime \prime} \nvdash^{\exists}$ $\overline{W^{\prime} \sim W^{\prime \prime}}$. Thanks to (1) and (2) we have the following: $X_{0} \subseteq W^{\prime}, X_{0} \subseteq W^{\prime \prime}$, $X_{1} \cap W^{\prime}=\varnothing$, and $X_{1} \cap W^{\prime \prime}=\varnothing$. Therefore $X_{0} \subseteq W^{\prime} \cap W^{\prime \prime}$ and $X_{1} \subseteq \bar{W}^{\prime} \cap \bar{W}^{\prime \prime}$, so from the fact that $W^{\prime} \sim W^{\prime \prime} \nvdash^{\exists} \overline{W^{\prime} \sim W^{\prime \prime}}$, alias $W^{\prime} \cap W^{\prime \prime}, \bar{W}^{\prime} \cap \bar{W}^{\prime \prime} \nvdash^{\exists} \overline{W^{\prime} \sim W^{\prime \prime}}$, we have, by (M): $X_{0}, X_{1} \nvdash^{\exists} \overline{W^{\prime} \sim W^{\prime \prime}}$ But from (1) and (2) again, it follows that while $y \in W^{\prime}$ (since $y \in X_{0}^{\prime} \subseteq W^{\prime}$ ), $y \notin W^{\prime \prime}$; so $y \in \overline{W^{\prime} \sim W^{\prime \prime}}$ and we have contradicted our original assumption that $X_{0}, X_{1} \vdash y$. 
Corollary 3.1.4. A Lindenbaum closure relation $\vdash$ satisfies $\left(\right.$ Flip-around ${ }^{\mathrm{cc}}$ ) iff $\vdash$ is determined by some class $\mathscr{V}$ of valuations such that $\mathscr{V} \sim \mathscr{V} \subseteq \mathscr{V}$ at $(\vdash)$.

Proof. Since the closure relations in question are, by Prop. 3.1.3, those satisfying (Match) $)_{2}$, we may take for $\mathscr{V}$ the characteristic functions of maximal avoiders, which $(\text { Match })_{2}$ says combine in pairs by $\sim$ to yield $\vdash$-closed sets, whose characteristic functions are valuations consistent with $\vdash$.

We are now in a position to eatablish that the conditions (Flip-around ${ }^{c c}$ ) and (Exchange) are not equivalent. We saw in Prop. 1.2.8 that the latter condition was satisfied by any closure relation satisfying the former. We show that the converse implication does not hold.

Proposition 3.1.5. Let $\vdash$ be the least closure relation on a set $U$ such that for all $x, y, z \in U$ with $x \neq y$, we have $x, y \vdash z$. Then $\vdash$ satisfies (Exchange).

Proof. Suppose $\vdash$ is as described but does not satisfy (Exchange), so that for some $X, x, y$ we have (1) $X, x \vdash y$ (2) $X, y \not \vdash x$ (3) $X \vdash y$. In virtue of $\vdash$ 's being a closure relation, (2) implies (4) $x \neq y$, while in virtue of the special condition imposed on $\vdash$, (2) implies that $X \cup\{y\}$ contains at most one element. Thus $X=$ $\varnothing$ or $X=\{y\}$; (3) rules out the latter possibility, so $X=\varnothing$, and (1) says that $x \vdash y$. But by (4), this is impossible, since $\vdash$ is the least closure relation satisfying the condition given.

Proposition 3.1.6. Let $U$ be any set containing at least four distinct elements $a$, $b, c, d$ and $\vdash$ be the least closure relation on $U$ such that $x, y \vdash z$ for all $x, y, z \in U$ with $x \neq y$. Then $\vdash$ does not satisfy $(\text { Match })_{2}$.

Proof. The maximal avoiders, relative to $\vdash$ as described, are the singleton subsets of $U$. The elements $c$ and $d$ belong to $\{a\} \sim\{b\}$, since $\{a\},\{b\}$ agree on not containing either of them, while $a \notin\{a\} \sim\{b\}$, since $\{a\},\{b\}$ differ in respect of $a$ 's membership. But $c$, $d \vdash a$, as $c \neq d$, so $\{a\} \sim\{b\}$ is not $\vdash$-closed, and accordingly $\vdash$ does not satisfy $\left(\right.$ Match $\left._{2}\right)$.

Corollary 3.1.7. A closure relation satisfying (Exchange) need not satisfy (Flip-around ${ }^{\mathrm{cc}}$ ).

Proof. Let $\vdash$ be a closure relation on $U$ meeting the conditions laid down in Prop. 3.1.6. Since $\vdash$ does not (by 3.1.6) satisfy $\left(\mathrm{Match}_{2}\right)$, it does not satisfy (Flip-around $^{\mathrm{cc}}$ ), the proof of Prop. 3.1.3 showing that all closure relations satisfying $\left(\right.$ Flip around ${ }^{\mathrm{cc}}$ ) satisfy $\left(\mathrm{Match}_{2}\right)$. But by Prop. 3.1.5, $\vdash$ does satisfy (Exchange). 
We can illustrate Coro. 3.1 .7 with a concrete case

Example 3.1.8. Let $U$ and $\vdash$ be as in Coro.3.1.7; we want $X_{0}, X_{1}, y$ with $X_{0}, X_{1} \vdash y$ but $X_{0}, y \not \nvdash^{c c} X_{1}$ and $X_{0} \not \nvdash^{c c} X_{1}, y$.

Take $X_{0}=\varnothing, X_{1}=\{a, b\}, y=c$.

We clearly have $X_{0}, X_{1} \vdash y$ for this case. To see that $X_{0}, y \not \nvdash^{\mathrm{cc}} X_{1}$, which is to say: $c \nvdash^{c c} a, b$, note that $c, a \vdash d$ and $c, b \vdash d$ while $c \not \nvdash d$. (Here we are taking $\{c\}$ itself as the relevant superse of $\{c\}$.) To see that $X_{0} \not \nvdash^{\mathrm{cc}} X_{1}, y$, i.e., $\forall{ }^{\mathrm{cc}} a, b, c$, observe that $d, a \vdash c$ and $d, b \vdash c$ and $d, c \vdash c$ while $d \nvdash c$. (Here $\{d\}$ is the relevant superset of $\varnothing$.

\subsection{An Unanswerd Question and a Further Condition}

Our discussion has left open the possibility that satisfaction (by a Lindenbaum closure relation) of (Flip-around ${ }^{\mathrm{cc}}$ ) is sufficient for determination by some class of valuations closed under $\sim$, a possibility deserving some comment. In view of Thm. 3.1.2 and Prop. 3.1.3, its realization would mean that the conditions (Match) ${ }_{n}$ for $n \geq 3$ follow from (Match) ${ }_{2}$. An attempt to prove this inductively meets with the following obstacle. Conditions such as (Match) ${ }_{2}$ with an $\vdash^{\exists}$-antecedent and a disjunctive $\vdash^{\text {cc }}$-consequent cannot be reapplied to their own outputs, since we are a given $\vdash^{\mathrm{cc}}$-statement is in general weaker than the corresponding $\vdash^{\exists}$-statement. A similar difficulty arises for principles like (Fliparound ${ }^{\exists c c}$ ) and (Flip-around ${ }^{c c}$ ), assuming in the latter case the following form: the antecedent is a straight $\vdash$-statement while the consequent's disjuncts are $\vdash{ }^{\mathrm{cc}}$-statements with, in general, multiple right-hand sides. To illustrate the difficulty:

Example 3.2.1. For any closure relation $\vdash$ determined by a class of valuations closed under $\sim$, the following condition is satisfied:

$\left(^{*}\right) \quad X_{0}, X_{1} \vdash y$ implies $X_{0}, y \vdash^{\mathrm{cc}} X_{1}$ or $X_{1}, y \vdash{ }^{\mathrm{cc}} X_{0}$ or $\vdash^{\mathrm{cc}} y, X_{0}, X_{1}$

We leave the reader to verify this, by consideration of the valuation $v_{1} \sim v_{2} \sim v_{3}$, where $v_{1}, v_{2}, v_{3}$ are valuations whose existence is called for by the assumption that each of the three disjuncts in the consequent of $\left(^{*}\right)$ is false.

In the special case of $\left(^{*}\right)$ in which $X_{1}=\{x\}$ :

(**) $\quad X_{0}, x \vdash y$ implies $X_{0}, y \vdash x$ or $x, y \vdash{ }^{\mathrm{cc}} X_{0}$ or $\vdash^{\mathrm{cc}} y, X_{0}, x$

we can derive the condition from (Flip-around ${ }^{\text {cc }}$ ) as follows: Given $X_{0}, x \vdash y$, we have $X_{0}, y \vdash x$ or $X_{0} \vdash{ }^{c c} x, y$, which latter simplifies by $(\mathbb{T})$, since we are given $X_{0}, x \vdash y$, to $X_{0} \vdash y$. (This is the Exchange Property, and we are running through the proof of Prop. 1.2.8 to highlight the role of $(\mathbb{T})$ for the sake of a remark below.) So it remains only to obtain the conclusion that either $x, y \vdash^{c c} X_{0}$ or $\vdash{ }^{c c} y, X_{0}, x$ 
from $X_{0} \vdash y$. But (Flip-around ${ }^{\mathrm{cc}}$ ) delivers from $X_{0} \vdash y$ the conclusion: $y \vdash^{\mathrm{cc}} X_{0}$ or $\vdash^{\mathrm{cc}} y, X_{0}$ (taking $X_{0}$ as $\varnothing \cup X_{0}$ ), from which the desired result follows by (M). In the general case of $\left({ }^{*}\right)$, however, this reasoning does not go through since we cannot apply $(\mathbb{T})$ when $X_{1}$ contains more than one element, so we cannot re-invoke the condition (Flip-around ${ }^{\text {cc }}$ ) with its single right-hand side.

Whether the obstacle here encountered can be circumvented gives us our

Unanswered Question: Can a Lindenbaum closure relation satisfy (Match) ${ }_{2}$ without satisfying $(\text { Match })_{n}$ for all $n>2$ ?

In other words, we are asking whether such a $\vdash$ could be found with $W \sim W^{\prime}$ $\vdash$-closed for all maximal avoiders, $W, W^{\prime}$ (relative to $\vdash$ ), without its being the case that for all maximal avoiders $W_{1}, \ldots, W_{n}$, we have $W_{1} \sim \ldots \sim W_{n} \vdash$-closed. A negative answer, if one could be obtained, would give a very attractive condition as necessary and sufficient for determination by a class of valuations closed under $\sim$, namely (in view of Prop. 3.1.3) the very simple condition (Flip-around ${ }^{c c}$ ) whose necessity in this respect was observed in Thm. 1.2.6. We return to this question (though without answering it) after discussing a condition which is in some respects an improvement on (Match), though not so much of an improvement as (Flip-around $^{\text {cc }}$ ) would be.

The difficulty to which Example 3.2.1 draws attention can be side-stepped by generalizing the condition $\left(^{*}\right)$ of the above Example, and replacing (Fliparound ${ }^{\text {cc }}$ ) by a condition (-schema) allowing a multiplicity of disjuncts in the consequent:

(Multi-flip) $X_{0}, \ldots, X_{n} \vdash y$ implies $\mathscr{A}_{1}$ or $\ldots$ or $\mathscr{A}_{\mathrm{k}}$

where each $\mathscr{A}_{j}$ is $a \vdash^{\mathrm{cc}}$-statement which some of the variables $X_{j}$ on the left of the ' $\vdash$ 'c ' and the rest on the right, with $y$ in each case on the left or the right, and with each $X_{j}$ appearing altogether an even number of times on the right, and $y$ appearing an odd number of times on the right.

In the case of $\left(^{*}\right), n=1, k=3$ and each of the $X_{j}$ appears twice on the right, with $y$ appearing once on the right. Other applications of (Multi-flip) will appear in our the argument for the 'only if' part of Thm. 3.2.2 below, which shows that (Mulyi-flip) is satisfied by the same Lindenbaum closure relation as (Match) is. As a condition on closure relations offered in solution to Problem 1.1.2, even setting aside the restriction to Lindenbaum closure relations, (Match) suffers from two inelegancies. The first is that it is an infinite collection-(Match) ${ }_{1}$, (Match) ...- of generalized rule-like conditions rather than a single such condition; the second is that it is formulated with the explicit help of the matching operation on subsets of $U$, as well as of complementation on these subsets. It would at least be of interest to see whether these ancillary set-theoretic devices could be 
dispensed with. (Naturally, since the commas on the left and right represent union, set-theoretic operations are not altogether dispensable: but by restricting ourselves to this one, we remain within the usual 'logical' tradition except to the extent that generalized rule-like conditions, rather than rule-like conditions proper, are being employed.) (Multi-flip) avoids this second inelegance, though of course it retains the first, since the schematic formulation subsumes infinitely many specific generalized rule-like conditions. (Of course, if the answer to our Unanswered Question above is negative, then we have, in (Flip-around ${ }^{\mathrm{cc}}$ ), a condition avoiding both inelegancies.) We have the same result for the new condition that we had for (Match) in Thm. 3.1.2:

Theorem 3.2.2. A Lindenbaum closure relation $\vdash$ satisfies (Multi-flip) iff $\vdash$ is determined by some class of valuations closed under $\sim$.

For the 'if' direction here, we reason as follows. The failure of one of the disjunctions $\mathscr{A}_{1}$ or $\ldots$ or $\mathscr{A}_{\mathrm{k}}$ in the consequent of an instance of (Multi-flip) would mean the existence of valuations $v_{1}, \ldots, v_{k}$ in any $\sim$-closed class of valuations $\mathscr{V}$ determining $\vdash$, with the requirements about the appearance of $X_{0}, \ldots, X_{n}, y$ on the left and right of ' $\vdash$ ' in this disjunction guaranteeing that $v_{1} \sim \ldots \sim v_{k}$ assigns $T$ to (every element of) each of the sets $X_{0}, \ldots, X_{n}$, and $\mathrm{F}$ to $y$ : so the antecedent of that instance of (Multi-flip) would also fail.

The 'only if' direction of Thm. 3.2.2 is established using the same class of valuations (for a given $\vdash$ ) as in the case of Thm. 3.1.2: characteristic functions of maximal avoiders and of sets $W_{1} \sim \ldots \sim W_{m}$ where each of the $W_{\imath}$ is a maximal avoider, and the work arises when it comes to showing that these matchcombinations of maximal avoiders are all $\vdash$-closed. Rather than doing this work in full generality, we illustrate the ideas involved with a representative case, in which $m=3$. Suppose that $\vdash$ satisfies (Multi-flip) and that $W_{1}, W_{2}, W_{3}$ are maximal avoiders (relative to $\vdash$ ) with $W_{1} \sim W_{2} \sim W_{3}$ not $\vdash$-closed, say because although

$$
W_{1} \sim W_{2} \sim W_{3} \vdash y
$$

we have

$$
y \notin W_{1} \sim W_{2} \sim W_{3}
$$

We need to derive a contradiction from (1) and (2), and we begin by re-writing (1) in a form amenable for treatment by (Multi-flip):

$$
W_{1} \cap W_{2} \cap W_{3}, \bar{W}_{1} \cap \bar{W}_{2} \cap W_{3}, \bar{W}_{1} \cap W_{2} \cap \bar{W}_{3}, W_{1} \cap \bar{W}_{2} \cap \bar{W}_{3} \vdash y
$$

Now since in general $y \notin W_{1} \sim \ldots \sim W_{k}$ means that $y$ fails to belong to an odd number of the $W_{i}$, there are two kinds of ways for (2) to be the case: $y$ is an element of exactly two of $W_{1}, W_{2}, W_{3}$ (being absent from 1 , that is), or else $y$ belongs to 
none of $W_{1}, W_{2}, W_{3}$ (being absent from 3 , that is). Let us take up the former possibility first, supposing, for definiteness, that

$$
y \in W_{1}, y \in W_{2}, y \notin W_{3} .
$$

Here the relevant disjunctive consequent of an instance of (Multi-flip) with

(3) as antecedent is: $\mathscr{A}_{1}$ or $\mathscr{A}_{2}$ or $\mathscr{A}_{3}$, where

$\mathscr{A}_{1}$ is: $W_{1} \cap W_{2} \cap W_{3}, W_{1} \cap \bar{W}_{2} \cap \bar{W}_{3}, y \vdash{ }^{\mathrm{cc}} \bar{W}_{1} \cap \bar{W}_{2} \cap W_{3}, \bar{W}_{1} \cap W_{2} \cap \bar{W}_{3}$

$\mathscr{A}_{2}$ is: $W_{1} \cap W_{2} \cap W_{3}, \bar{W}_{1} \cap W_{2} \cap \bar{W}_{3}, y \vdash{ }^{c c} \bar{W}_{1} \cap \bar{W}_{2} \cap W_{3}, W_{1} \cap \bar{W}_{2} \cap \bar{W}_{3}$

$\mathscr{A}_{3}$ is; $W_{1} \cap W_{2} \cap W_{3}, \bar{W}_{1} \cap \bar{W}_{2} \cap W_{3} \vdash^{\mathrm{cc}} y, \bar{W}_{1} \cap W_{2} \cap \bar{W}_{3}, W_{1} \cap \bar{W}_{2} \cap \bar{W}_{3}$

The recipe followed here for obtaining $\mathscr{A}_{i}$ collects on the left all the intersection terms in which $W_{i}$ figures, and, on the right, the remaining intersection terms, in which $\bar{W}_{i}$ figures instead, and puts $y$ on the left or the right according as $y \in W_{i}$ or $y \notin W_{i}$, respectively. Thus, in view of (4), $y$ appears on the right only in $\mathscr{A}_{3}$, since the only one of $W_{1}, W_{2}, W_{3}$, not containing $y$ is $W_{3}$.

This then allows us to argue, much as in the 'if' part of the proof of Prop. 3.1.4, as follows: from $\mathscr{A}_{1}$ we infer (by $(\mathbb{M})$ ) that $W_{1}, W_{1}, y \vdash^{\mathrm{cc}} \bar{W}_{1}, \bar{W}_{1}$, i.e., $W_{1}, y \vdash^{\text {cc }} \bar{W}$, which is to say (since $y \in W_{1}$ ) $W_{1} \vdash^{c c} \bar{W}_{1}$, in contradiction to Prop. 0.5 .1 (ii). From $\mathscr{A}_{2}$ we similarly obtain $W_{2}, y \vdash^{\mathrm{cc}} \bar{W}_{2}$ hence, as $y \in W_{2}: W_{2} \vdash^{c c} \bar{W}_{2}$, which is likewise impossible. From $\mathscr{A}_{3}$ we reason to $W_{3} \vdash^{\text {cc }} y, \bar{W}_{3}$ and hence, since $y \notin W_{3}$, to the similarly impossible conclusion that $W_{3} \vdash^{c c} \bar{W}_{3}$. Thus (1) and (4) cannot be true together for $\vdash$ satisfying (Multi-flip). What we had to show was that this was so for (1) and (2), with (4) representing one way for (2) to obtain. As remarked above, the other way is for us to have:

$$
y \notin W_{1}, y \notin W_{2}, y \notin W_{3} .
$$

but this leads, when the recipe described above is followed, to a choice of $\mathscr{A}_{1}$ or $\mathscr{A}_{2}$ or $\mathscr{A}_{3}$ like that of the preceding case except that $y$ appears on the right in all three disjuncts, giving us a contradiction in each case by the reasoning employed a propos of the $\mathscr{A}_{3}$ displayed above.

We will close our discussion by considering our 'unanswered question' in the context of finitary closure relations, where it receives a rather clear articulation given the analysis in terms of tight sets provided by 0.6 .

Lemma 3.2.3. If $X_{0}$ is a tight set relative to any Lindenbaum closure relation $\vdash$ and $a, b$ are any two elements of $X_{0}$, then there is a maximal avoider containing all elements of $X_{0} \backslash\{a, b\}$ but neither of $a, b$.

Proof. If $X$ is tight then $X_{0} \backslash\{a, b\} \not \forall b$, so there is a maximal avoider extending $X_{0} \backslash\{a, b\}$ and not containing $b$; since $X_{0} \backslash\{a, b\}, a \vdash b$, this maximal avoider also fails to contain $a$. 
Lemma 3.2.3 offers a version of what Lemma 1.2.2 said about generalized closure relations. We used Lemma 1.2.2 to prove a result (Thm. 1.2.3) showing that for any generalized closure relation satisfying (Flip-around) and with respect to which $X \cup Y$ is tight and $|Y|$ is odd, we must have $X \vdash Y$. The current analogue of this result would be one affirming that any Lindenbaum closure relation satisfying (Flip-around ${ }^{\text {cc }}$ ) and w.r.t. which $X \cup Y$ is tight with $|Y|$ odd, we have $X \vdash^{\mathrm{cc}} Y$. But the inductive part of the proof of Thm. 1.2.3 used and inductive hypothesis to the effect that, $X, a, b \vdash Y$ and reasoned by appeal to (Flip-around) that either $X, Y \vdash a, b$ or else $X \vdash Y, a, b$, the first of which possibilities was ruled out by Lemma 1.2.2. Superscripting these $\vdash$-statements with 'cc', we have the first possibility again ruled out - this time by Lemma 3.2.3 (and Prop. 0.5.1 (iv)) - but the analogous appeal to (Flip-around ${ }^{\mathrm{cc}}$ ) in deriving this disjunction of possibilities is blocked since we cannot invoke the latter condition for an assumption of the form $X, a, b \vdash^{c c} Y$ unless $|Y|=1$, so that the 'cc' is redundant. Thus the induction does not get very far for the present case, and we have only: if $X \cup Y$ is a tight set relative to a Lindenbaum closure relation satisfying (Flip-around ${ }^{c c}$ ), then $X \vdash^{c c} Y$ for $Y$ with $|Y|=1$ or 3 . Let us say that a maximal avoider $W$ omits $x$ when $x \notin W$. Then we can rephrase our conclusion as

Proposition 3.2.4. Relative to a Lindenbaum closure relation satisfying (Fliparound $\left.^{\mathrm{cc}}\right)$, no maximal avoider can omit exactly 1 or exactly 3 elements of a tight set.

This limited conclusion explains the highly conditional nature of the following:

Lemma 3.2.5. For all $n \geq 1$, if $\vdash$ is a closure relation for which $W_{1} \sim \ldots \sim W_{n}$ is always $\vdash$-closed when $W_{1}, \ldots, W_{n}$ are maximal avoiders, then no maximal avoider (relative to $\vdash$ ) can omit precisely $2 n+1$ elements of any tight set.

Proof. Suppose the conditions of the Lemma are met by a closure relation $\vdash$ but (for a contradiction) that there is some maximal avoider $W$ which omits from some tight set $X$ precisely the $2 n+1$ (distinct) elements $a_{1}, \ldots, a_{2 n+1}$. Write $X$ as $Y \cup\left\{a_{1}, \ldots, a_{2 n+1}\right\}$, with none of the $a_{i} \in Y$. By Lemma 3.2.3 we have maximal avoiders omitting each pair $\left\{a_{1}, a_{2}\right\},\left\{a_{3}, a_{4}\right\}, \ldots,\left\{a_{2 n-1}, a_{2 n}\right\}$ and (in each case) no other elements of $X$. Call them respectively $W_{1,2}, W_{3,4}, \ldots, W_{2 n-1,2 n}$, and note that they number $n-1$ altogether. Thus by the condition laid down for $\vdash$ in the Lemma, the set $W \sim W_{1,2} \sim W_{3,4} \sim \ldots \sim W_{2 n-1,2 n}$ should be $\vdash$-closed. But this set includes $Y$ (since all its 'factors' do) and contains all of $a_{1}, \ldots, a_{2 n}$, (since $a_{1}, a_{2}$ are missing from $W_{1,2}$ and $W$ but present in all the other factors, and similarly for $a_{3,}$ $a_{4}$, etc.) without containing $a_{2 n \vdash 1}$, as this element is missing only from $W$. This contradicts the tightness of $X$, since the latter implies, in particular, that $Y, a_{1}, \ldots, a_{2 n} \vdash a_{2 n+1}$. 
Theorem 3.2.6. Let $\vdash$ be a finitary closure relation satisfying (Exchange). Then all sets of the form $W_{1} \sim \ldots \sim W_{n}$ (the $W_{i}$ being maximal avoiders) are $\vdash$-closed if and only no maximal avoider omits an odd number of elements from any tight set.

Proof. 'Only if': This direction does not even require the hypotheses regarding finitariness and the Exchange Property, since it follows directly from Lemma 3.2.5 for arbitrary $\vdash$.

'If': Suppose that $\vdash$ is a finitary closure relation satisfying (Exchange) and $W_{1} \sim \ldots \sim W_{n} \vdash y$; we must show that if no maximal avoider relative to $\vdash$ omits an odd number of elements from any tight set, then $y \in W_{1} \sim \ldots \sim W_{n}$. By Prop. 0.6 .1 and the fact that $\vdash$ is finitary we have some finite $X \subseteq W_{1} \sim \ldots \sim W_{n}$ with $X \cup\{y\}$ tight. Write $X \cup\{y\}$ as $\left\{x_{1}, \ldots, x_{k}\right\}$ where the $x_{\imath}$ are all distinct ( $x_{k}$ being $y$, say). Consider the $k$-by- $n$ rectangular array with columns headed by $W_{1}, \ldots, W_{n}$ and rows headed by $x_{1}, \ldots, x_{k}$ with a ' + ' in position $a_{\imath j}$ if $x_{i} \in W_{\jmath}$ and a $\because$ there otherwise. Since for each $i<k, x_{i} \in W_{1} \sim \ldots \sim W_{n}$, there are an even number of '-'s in each of the rows headed by these $x_{i}$, while if (for a contradiction) $y=x_{k} \notin W_{1} \sim \ldots \sim W_{n}$, there are an odd number of '-'s in the bottom (that is, the $\left.k^{\text {th }}\right)$ row. Therefore, adding the numbers of ' ' in all the rows gives an odd number of '-'s in the whole array. So at least one $W_{j}$ must head a column containing an odd number of '-'s, which is impossible on the assumption that no maximal avoider omits an odd number of elements from any tight set.

With regard to the claim, mentioned above as giving a negative answer to our 'unanswered question', that if for all maximal avoiders, $W, W$ ', relative to (some Lindenbaum closure relation) $\vdash$, we have $W \sim W^{\prime} \vdash$-closed, then for all maximal avoiders $W_{1}, \ldots, W_{n}$, we must have $W_{1} \sim \ldots \sim W_{n} \vdash$-closed, we have the following situation. The simplest possible counterexample to the claim would consist in exhibiting a $\vdash$ for which all matches of pairs of maximal avoiders are $\vdash$-closed, but for which there is a maximal abvoider omitting precisely 5 elements from some set tight relative to $\vdash$. (The smaller odd cases were ruled out by Prop. 3.2.4.) However, the author has been able neither to prove that for the Lindenbaum closure relations concerned - those satisfying (Flip-around ${ }^{\mathrm{cc}}$ ) or equivalently $(\mathrm{Match})_{2}$-no maximal avoider can omit precisely 5 elements from any tight set, nor to construct an example of such a $\vdash$ with a maximal avoider which does omit 5 elements from a tight set. While finding such an example would definitively return a positive to our unanswered question, showing that Problem 1.1.2 requires a more complex condition such as (Match) or (Multi-flip), showing that no such case exists would not of course automatically give a negative answer to that question, even for the case of finitary $\vdash$; it would, however, be highly surprising if any proof that none of the $\vdash$ with which are concerned had maximal avoiders omitting exactly 5 elements from a tight set left this as an open possibility for 7,9 , ... such elements. 
The question we are leaving unanswered here is a special case of the general question raised in 2.5 by the discussion leading up to and immediately following Prop. 2.5.10 and Coro. 2.5.11: the extent to which the ' $\exists \mathrm{cc}$ ' strategy issues in success. Since (Flip-around ${ }^{\mathrm{cc}}$ ) is equivalent to the result, (Flip-around ${ }^{\exists c c}$ ), of applying this strategy, as well as - for Lindenbaum closure relations - to (Match) ${ }_{2}$, Thm.3.2.1 tells us that the strategy is successful in this instance just in case for all $n>2$, (Match) $)_{n}$ must be satisfied by every such closure relation satisfying $(\text { Match })_{2}$.

\section{References}

[1] Belnap, N.D. and Massey, G.J. Semantic Holism, Studia Logica, 49 (1990), 67-82.

[2] Birkhoff, G., Lattice Theory (3rd Edn.), American Math. Soc. Colloquium Publications, 25, American Math. Soc., Providence, Rhode Island 1967 (1st Edn. 1940).

[3] Carnap, R., Formalization of Logic, reprinted in Introduction to Semantics and Formalization of Logic, Harvard University Press 1961 (Orig. publ.1943).

[4] Dénes, J., and Keedwell, A.D., Latin Squares and their Applications, Academic Press (New York and London), 1974.

[5] Došen, K., and Schroeder-Heister, P., Uniqueness, Definability and Interpolation, Journal of Symbolic Logic, 53 (1988), 554-570.

[6] van Fraassen, B.C., Formal Semantics and Logic, Macmillan 1971.

[ 7 ] Gabbay, D., Semantical Investigations in Heyting's Intuitionistic Logic, Reidel, Dordrecht 1981.

[ 8 ] - What is a Classical Connective?, Z. Math. Logik Grundlag Math., 24 (1978), 37-44.

[9] Garson, J. W., Categorical Semantics, J. M. Dunn and A. Gupta (eds.) Truth or Consequences: Essays in Honor of Nuel Belnap, Kluwer Academic Publishers, Dordrecht (1990), 155-175.

[10] Gierz, G., Hofmann, K. H., Keimel, K., Lawson, J. D., Mislove, M. and Scott, D. S., $A$ Compendium of Continuous Lattices, Springer-Verlag, 1980.

[11] Głazek, K., Some Old and New Problems in Independence Theory, Coll. Math., 42 (1979), 118-189.

[12] Herrlich, H. and Hušek, M., 'Galois Connections', A. Melton (ed.), Mathematical Foundations of Programming Semantics, Lecture Notes in Computer Science, SpringerVerlag, 239 (1986), 122-134.

[13] Humberstone, I. L., A Study of some "Separated" Conditions on Binary Relations, Theoria, 57 (1991), 1-16.

[14] - Zero-Place Operations and Functional Completeness, History and Philos. Logic, 14 (1993), 39-66.

[15] - Functional Dependencies, Supervenience, and Consequence Relations, J. Logic, Language and Information, 2 (1993), 309-336.

[16] Kim, J., Concepts of Supervenience, Philosophy and Phenomenological Research, 48 (1984), 153-176.

[17] Lewis, D., On the Plurality of Worlds, Basil Blackwell, Oxford, 1986.

[18] Mac Lane, S., Categories for the Working Mathematician, Springer-Verlag, Berlin, 1971.

[19] McKee, T. A., Generalized Equivalence: A Useful Pattern of Mathematical Expression, Studia Logica, 44 (1985), 285-289.

[20] Ore, O., Galois Connexions, Trans. Amer. Math. Soc., (1944), 493-513.

[21] Post, E. L., The Two-Valued Iterative Systems of Mathematical Logic, Princeton University Press, 1941.

[22] Scott, D. S., Rules and Derived Rules, S. Stenlund (ed.), Logical Theory and Semantic Analysis, 147-161, Reidel, Dordrecht 1974. 
[23] Scott, D. S., Completeness and Axiomatizability in Many-Valued Logic, L. Henkin et al. (eds.), Procs. of the Tarski Symposium, American Math. Society, Providence, Rhode Island, 1974.

[24] Segerberg, K., Classical Propositional Operators, Clarendon Press, Oxford, 1982.

[25] - Arbitrary Truth-Value Functions and Natural Deduction, Z. Math. Logik Grundlag. Math., 29 (1983), 557-564.

[26] Shoesmith, D. J., and T. J. Smiley, Multiple-Conclusion Logic, Cambridge University Press, Cambridge 1978.

[27] Surma, S. J., Remarks on the Consistency-Theoretic versus Proof-Theoretic Approaches to Metalogic and Metamathematics, Unpublished Typescript, 1992.

[28] Wójcicki, R., Theory of Logical Calculi, Kluwer Academic Publishers, Dordrecht, 1988.

[29] Wojtylak, P., Independent Axiomatizability of Sets of Sentences, Ann. Pure Appl. Logic, 44 (1989), 259-299. 